\title{
Game-Theoretic Upper Expectations for Discrete-Time Finite-State Uncertain Processes
}

\author{
Natan T'Joens, Jasper De Bock, Gert de Cooman \\ FLip, Ghent University, Belgium \\ Technologiepark-Zwijnaarde 125, 9052 Zwijnaarde
}

\begin{abstract}
Game-theoretic upper expectations are joint (global) probability models that mathematically describe the behaviour of uncertain processes in terms of supermartingales; capital processes corresponding to available betting strategies. Compared to (the more common) measure-theoretic expectation functionals, they are not bounded to restrictive assumptions such as measurability or precision, yet succeed in preserving, or even generalising many of their fundamental properties. We focus on a discrete-time setting where local state spaces are finite and, in this specific context, build on the existing work of Shafer and Vovk, the main developers of the framework of game-theoretic upper expectations. In a first part, we study Shafer and Vovk's characterisation of a local upper expectation and show how it is related to Walley's behavioural notion of coherence. The second part consists in a study of game-theoretic upper expectations on a more global level, where several alternative definitions, as well as a broad range of properties are derived, e.g. the law of iterated upper expectations, compatibility with local models, coherence properties, ... Our main contribution, however, concerns the continuity behaviour of these operators. We prove continuity with respect to non-increasing sequences of so-called lower cuts and continuity with respect to non-increasing sequences of finitary functions. We moreover show that the game-theoretic upper expectation is uniquely determined by its values on the domain of bounded below limits of finitary functions, and show in addition that, for any such limit, the limiting sequence can be constructed in such a way that the game-theoretic upper expectation is continuous with respect to this particular sequence.
\end{abstract}

Keywords: Game-theoretic probability, Upper expectation, Coherence, Continuity properties, Finitary function

\section{Introduction}

We consider sequences $X_{1}, X_{2}, \ldots, X_{n}, \ldots$ of uncertain states where the state $X_{k}$ at each discrete time $k \in \mathbb{N}$ takes values in a fixed finite state space $\mathscr{X}$. The uncertain evolution of the state $X_{k}$ in such a sequence-which we will call a discrete-time finite-state uncertain process - can be described mathematically in various ways. Most authors prefer a measure-theoretic approach, which involves the use of countably additive probability measures. In this paper, we consider an alternative, game-theoretic approach that uses (super)martingales as a primitive notion. Probabilities and expectations then only appear as derived, secondary objects. This approach was largely developed by Shafer and Vovk [12, 13], but some of the essential underlying ideas are due to Ville [22].

Irrespectively of the chosen mathematical framework, the starting point for modelling an uncertain process is typically a set of assessments about the local uncertain behaviour of the process. Such local assessments

Email address: natan.tjoens@ugent. be (Natan T'Joens) 
represent our beliefs on how the state $X_{k}$ of the process will change from one time instant to the next. In a traditional approach, they are given in the form of probabilities $\mathrm{P}\left(X_{k+1}=x_{k+1} \mid X_{1}=x_{1}, \cdots, X_{k}=x_{k}\right)$ for each possible history $X_{1}=x_{1}, \cdots, X_{k}=x_{k}$ (with $x_{i} \in \mathscr{X}$ ) and for each possible value $x_{k+1} \in \mathscr{X}$ for the next state $X_{k+1}$. However, when information is scarce or when we want to act conservatively, specifying such a single probability measure for each history might not be appropriate or not even justified. In such cases, our beliefs can be more accurately described by imprecise probabilities models [1, 24], e.g. probability intervals, sets of probability charges (or measures), sets of desirable gambles or upper and lower expectations. Each of these models allows us to express and incorporate partial knowledge about the parameters that would normally make up a traditional precise probability model. We will here focus on one particular-yet general and unifying-such imprecise probabilities model: upper and lower expectations (or previsions).

Upper and lower expectations generalise traditional expectation operators in the sense that the additivity property is replaced by the weaker condition of sub- or super-additivity, respectively. A typical interpretation for them is that they are respective upper and lower bounds on a set of plausible expectations. In fact, it can be shown that they are in a one-to-one relation with closed, convex sets of linear expectations [24] or, equivalently, closed convex sets of probability charges or measures. Interval probabilities therefore only correspond to a special case. Walley [24], on the other hand, uses a behavioural interpretation where bounded real-valued functions on a non-empty set $\mathscr{Y}$ represent gambles with an uncertain payoff and where the upper (or lower) expectation $\overline{\mathrm{E}}(f)$ (or $\underline{\mathrm{E}}(f)$ ) of such a gamble $f$ represents a subject's infimum selling price (or supremum buying price) for $f$. By imposing that a subject should gamble rationally - which he calls 'coherence'-Walley then obtains the same axioms as those of a sub- or superadditive expectation operator. Walley's interpretation moreover naturally connects upper and lower expectations with another type of imprecise probabilities model, called 'sets of desirable gambles', which models uncertainty by considering the gambles a subject finds desirable. Due to its different interpretations and its connections with various other (imprecise) uncertainty models, the theory of upper and lower expectations has a unifying role within the field of imprecise probabilities, hence our choice for using them here to model uncertainty in stochastic processes. More detailed information about lower and upper expectations can be found in References [7, 24].

So suppose that, for each possible history $X_{1}=x_{1}, \cdots, X_{k}=x_{k}$, we are given an upper expectation $\overline{\mathrm{Q}}_{x_{1 \cdot k}}$ that expresses our beliefs about the value of the next state $X_{k+1}$. How can we use these assessments to draw conclusions about more general behaviour of the process? For instance, what is the probability that the process state will ever be equal to a particular value $x \in \mathscr{X}$ ? When do we expect this to happen for the first time? Such inferences relate to the value of the process state at multiple time instances. To make statements about them, we need a global uncertainty model that incorporates and extends the information included in the local uncertainty models $\overline{\mathrm{Q}}_{r_{1, j}}$. One particularly interesting way of constructing such a global model was proposed by Shafer and $\operatorname{Vovk}[12,13]$.

They picture a game that consists of a (possibly infinite) series of rounds and three players, called Forecaster, Skeptic and Reality. In the beginning of each round, Forecaster declares how he is willing to gamble on the outcome of the current round. Subsequently, Skeptic takes him up on his commitments and chooses a gamble from the ones offered by Forecaster. At the end of the round, Reality decides the outcome and Forecaster and Skeptic exchange money accordingly. Now, given Forecaster's assessments, how should Skeptic determine a 
selling (or buying) price for a gamble $f$ whose uncertain payoff depends on an entire realisation $\omega$-which may take an infinitely long time—of the game? Shafer and Vovk argue that Skeptic should certainly agree on selling the gamble $f$ for any price $\alpha$ such that there is a gambling strategy for Skeptic that starts with an initial capital $\alpha$ and allows him to end up with at least as much money as the payoff $f(\omega)$ corresponding to $f$, irrespective of the realisation $\omega$ of the game. The infimum of all such prices $\alpha$, which depends entirely on Forecaster's local assessments, is then what Shafer and Vovk call the game-theoretic upper expectation of $f$. Game-theoretic lower expectations are subsequently defined in an analogous, yet dual way.

In the setting that we consider, Forecaster's local assessments are modelled by the local upper expectations $\overline{\mathrm{Q}}_{x_{1: k}}$ and the allowed gambles for Skeptic are those functions $f$ on $\mathscr{X}$ for which $\overline{\mathrm{Q}}_{x_{1: k}}(f) \leq 011$ The evolution of Skeptic's capital corresponding with an allowable betting strategy is called a supermartingale. As sketched above, these supermartingales can be used to define (global) game-theoretic upper and lower expectations. Mathematically speaking, these operators have the advantage of being very general, in the sense that they do not rely on an assumption of measurability or on an assumption that uncertainty should be modelled through traditional (precise) probability models. Despite their generality, they also possess rather strong technical properties, and therefore maintain great practical usefulness. Though many slightly different definitions of the game-theoretic upper expectation can be found throughout the literature, the key elements always remain the same. We consider one particular definition here, since we believe it to have, in our setting, superior mathematical properties compared to other versions; we will argue so in Section 8, Moreover, the version we consider can also be elegantly characterised as the most conservative uncertainty model that satisfies a set of intuitive axioms, and its use can therefore also be motivated without relying on an interpretation in terms of supermartingales or other game-theoretic concepts [18, 19].

The contribution of this paper is fourfold, yet serves the general aim of strengthening the theory and relevance of game-theoretic upper expectations for the case where the state space $\mathscr{X}$ is finite.

Our first contribution consists in bringing clarity about a number of properties that were already proved elsewhere for game-theoretic upper expectations, but usually in a slightly other setting or for a slightly other version of its definition. This concerns in particular the more basic properties (e.g. compatibility with local models, law of iterated upper expectations, ...) in Section 4 the equivalent definitions given in Section 5 and the non-decreasing continuity property in Section [6 Since it is our aim to bring clarity concerning these properties, we give self-contained proofs for these results. We will state explicitly when we borrow ideas from other work.

Our second contribution consists in showing that-for a finite state space $\mathscr{X}$-our version of the gametheoretic upper expectation also satisfies several new continuity properties. Such properties are powerful mathematical tools that provide the resulting theory with elegance and also greatly enhance its practical scope. The continuity of the Lebesgue integral, for instance, is one of the reasons why it is the integral of choice for computing expected values associated with a probability measure [2, 15]. We show in Section [6t that game-theoretic upper expectations satisfy continuity with respect to non-decreasing sequences (as pointed out in the previous paragraph, this property is not entirely new), continuity with respect to non-increasing sequences of lower cuts and a version of Fatou's lemma. In Section 7 we focus on continuity with respect to sequences of so-called fi-

\footnotetext{
${ }^{1}$ Note that there is a close relation with Walley's interpretation for upper expectations here; we refer to [5] for an extensive study.
} 
nitary functions. Such finitary functions only depend on the process state at a finite number of time instances. They can be given a clear interpretation [18, 19] and their upper expectations can often be calculated in a straightforward manner [10,21]. And although many practically relevant functions are themselves not finitary, most of them can still be written as the (pointwise) limit of a sequence of finitary functions [9, 21], e.g. hitting times, hitting probabilities, ... We prove continuity with respect to non-increasing sequences of finitary functions, and show that for any bounded below (pointwise) limit of finitary functions, the limiting sequence can be constructed in such a way that the game-theoretic upper expectation is continuous with respect to this particular sequence. Finally, we will also show that the game-theoretic upper expectation is uniquely determined by its values on the domain of all bounded below (pointwise) limits of finitary functions.

Our third contribution — which will be the topic of Section_2and, to a small extent, also of Section [6 —consists in bridging part of the gap that currently exists between what Shafer and Vovk do, and the work on upper and lower expectations (or previsions) in the field of imprecise probabilities. The latter is often based on Walley's notion of coherence which only considers upper (and lower) expectations on bounded real-valued functions. In such a context, the connection with Shafer and Vovk's earlier work was already studied elaborately in [5]. Here, we deal with extended real-valued functions and study how both approaches are related on a local level. We first propose a slightly weaker-and therefore, more general-version of Shafer and Vovk's axioms for a local upper expectation and show that, for a finite state space, this weaker version can equivalently be obtained by combining coherence (on bounded real-valued functions) with an upward continuity axiom. This result shows that the local upper expectations defined in this way are, on the domain of bounded below extended real-valued functions, uniquely determined by their values on bounded real-valued functions. This allows us to justify the use of our local models (on the domain of bounded below functions) - and therefore, also the use of the global gametheoretic upper expectations that are derived from them-from a more conventional imprecise probabilities point of view [19]. Moreover, by the end of Section [6 it will become clear that a particular downward continuity axiom needs to be satisfied by the local upper expectations in order for them to be (completely) compatible with the global game-theoretic upper expectation. This axiom will generally not be satisfied, even if we use the stronger axiomatisation for local upper expectations proposed by Shafer and Vovk; see Section 2. Hence, if we desire compatibility of local and global models, we will need to additionally impose the downward continuity axiom.

Our fourth and final contribution can be found in Section 8, where we compare different definitions of the game-theoretic upper expectation and argue why we have chosen to work with the particular version considered here. We take into account not only technical aspects, but also interpretational aspects of the possible definitions. We feel the need to include such a discussion here, because, as already mentioned, many different definitions have been used in the literature and it is often unclear-especially for an unexperienced reader-how these different versions relate to each other. We moreover show there that our particular definition of the gametheoretic upper expectation can be replaced by a more intuitive one, if we restrict ourselves to bounded realvalued functions.

In short, the outline of the paper is as follows: Section 2 shows how upper expectations on a finite state space can be equivalently axiomatised, either in a way similar to what Shafer and Vovk propose, or using Walley's concept of coherence together with a continuity axiom. In Section 3 , we use the upper expectations of Section 2 
as local uncertainty models in a process and show how these can then be used to define supermartingales and a global game-theoretic upper expectation. Sections 47 then focus on the mathematical properties of this global operator. We conclude the article in Section 8 with a discussion of other possible versions of game-theoretic upper expectations.

\section{Upper Expectations}

We consider and relate two possible approaches to characterising upper expectations on extended real-valued functions: one similar to Shafer and Vovk's axiomatisation, and one that uses Walley's notion of coherence in combination with an upward continuity axiom. Since we will later on use these characterisations to define local upper expectations in uncertain processes with a finite state space, we will mainly focus on extended real-valued functions with finite domain here. We show that, in this case, both characterisations are equivalent. Moreover, it will turn out that the characterising axioms of an upper expectation are also satisfied by the global gametheoretic upper expectation that we will consider further on, which indeed allows us to call this global operator an upper expectation.

We start by introducing some preliminary notions. We denote the set of all natural numbers, without 0 , by $\mathbb{N}$, and let $\mathbb{N}_{0}:=\mathbb{N} \cup\{0\}$. The set of extended real numbers is denoted by $\overline{\mathbb{R}}:=\mathbb{R} \cup\{+\infty,-\infty\}$. The set of positive real numbers is denoted by $\mathbb{R}_{>0}$, the set of non-negative real numbers by $\mathbb{R}_{\geq 0}$, and we also let $\overline{\mathbb{R}}_{>0}:=\mathbb{R}_{>0} \cup\{+\infty\}$ and $\overline{\mathbb{R}}_{\geq 0}:=\mathbb{R}_{\geq 0} \cup\{+\infty\}$. We extend the total order relation $<$ on $\mathbb{R}$ to $\mathbb{R}$ by positing that $-\infty<c<+\infty$ for all $c \in \mathbb{R}$ and endow $\overline{\mathbb{R}}$ with the associated order topology.

For any non-empty set $\mathscr{Y}$, a variable $f$ on $\mathscr{Y}$ is a map on $\mathscr{Y}$. A real(-valued) variable is a variable taking values in the reals $\mathbb{R}$, and similarly for an extended real(-valued) variable. We say that an extended real variable $f$ is bounded below if there is an $M \in \mathbb{R}$ such that $f \geq M$-meaning that $f(y) \geq M$ for all $y \in \mathscr{Y}$ 一and bounded above if $-f$ is bounded below. A gamble on $\mathscr{Y}$ is a real variable on $\mathscr{Y}$ that is bounded, meaning that it is both bounded below and bounded above. The set of all extended real variables on $\mathscr{Y}$ is denoted by $\overline{\mathscr{L}}(\mathscr{Y})$, the set of all bounded below extended real variables on $\mathscr{Y}$ by $\overline{\mathscr{L}}_{b}(\mathscr{Y})$ and the linear space of all gambles on $\mathscr{Y}$ by $\mathscr{L}(\mathscr{Y})$. For any $f \in \overline{\mathscr{L}}(\mathscr{Y})$ we use $\sup f$ and $\inf f$ to denote the supremum $\sup \{f(y): y \in \mathscr{Y}\}$ and $\operatorname{infimum} \inf \{f(y): y \in \mathscr{Y}\}$ of the variable $f$, respectively. We say that a sequence $\left\{f_{n}\right\}_{n \in \mathbb{N}_{0}}$ in $\overline{\mathscr{L}}(\mathscr{Y})$ is uniformly bounded below if there is an $M \in \mathbb{R}$ such that $f_{n} \geq M$ for all $n \in \mathbb{N}_{0}$. For any sequence $\left\{f_{n}\right\}_{n \in \mathbb{N}_{0}}$ in $\overline{\mathscr{L}}(\mathscr{Y})$, we write $\lim _{n \rightarrow+\infty} f_{n}$ to mean the pointwise limit of the functions $f_{n}$. For a subset $A$ of $\mathscr{Y}$, we define the indicator $\mathbb{I}_{A}$ of $A$ as the gamble on $\mathscr{Y}$ that assumes the value 1 on $A$ and 0 elsewhere.

\subsection{Upper expectations according to Shafer and Vovk}

The following definition of an upper expectation is very similar to what Shafer, Vovk and Takemura call a 'superexpectation' in [14] and an 'upper expectation' in [13] 2

Definition 1. Consider any non-empty set $\mathscr{Y}$ and any $\overline{\mathscr{L}}_{b}(\mathscr{Y}) \subseteq \mathscr{D} \subseteq \overline{\mathscr{L}}(\mathscr{Y})$. Then we define an upper expectation $\overline{\mathrm{E}}$ on $\mathscr{D}$ as an extended real-valued map on $\mathscr{D}$ that satisfies the following axioms:

\footnotetext{
${ }^{2}$ Their definition is, as far as we know, not based on a single interpretation. Rather, they draw inspiration from various subfields in probability theory to obtain these axioms.
} 
E1. $\overline{\mathrm{E}}(c)=c$ for all $c \in \mathbb{R}$;

E2. $\overline{\mathrm{E}}(f+g) \leq \overline{\mathrm{E}}(f)+\overline{\mathrm{E}}(g)$ for all $f, g \in \overline{\mathscr{L}}_{b}(\mathscr{Y})$;

E3. $\overline{\mathrm{E}}(\lambda f)=\lambda \overline{\mathrm{E}}(f)$ for all $\lambda \in \mathbb{R}_{>0}$ and all $f \in \overline{\mathscr{L}}_{b}(\mathscr{Y})$;

E4. $f \leq g \Rightarrow \overline{\mathrm{E}}(f) \leq \overline{\mathrm{E}}(g)$ for all $f, g \in \overline{\mathscr{L}}_{b}(\mathscr{Y})$.

E5. $\lim _{n \rightarrow+\infty} \overline{\mathrm{E}}\left(f_{n}\right)=\overline{\mathrm{E}}\left(\lim _{n \rightarrow+\infty} f_{n}\right)$ for any non-decreasing sequence $\left\{f_{n}\right\}_{n \in \mathbb{N}_{0}}$ ofnon-negative variables in $\overline{\mathscr{L}}_{b}(\mathscr{Y})$.

Alternatively, we can also consider the so-called conjugate lower expectation $\underline{\mathrm{E}}$, defined by $\underline{\mathrm{E}}(f):=-\overline{\mathrm{E}}(-f)$ for all $f \in-\mathscr{D}:=\{f \in \overline{\mathscr{L}}(\mathscr{Y}):-f \in \mathscr{D}\}$. It clearly suffices to focus on only one of the two functionals and we will work mainly with upper expectations. Furthermore, in the definition above, as well as further on, we adopt the following conventions: $c+\infty=+\infty$ for all real $c,+\infty+\infty=+\infty, \lambda(+\infty)=+\infty$ for all $\lambda \in \overline{\mathbb{R}}_{>0}$ and $0(+\infty)=0$. In addition to E1 E5, we will sometimes also impose 'continuity with respect to lower cuts' (also called 'bounded-below support') on an upper expectation. To introduce this property, let $f^{\vee c}$ for any $f \in \overline{\mathscr{L}}(\mathscr{Y})$ and any $c \in \mathbb{R}$ be the variable defined by $f^{\vee c}(y):=\max \{f(y), c\}$ for all $y \in \mathscr{Y}$. An upper expectation $\overline{\mathrm{E}}$ on $\mathscr{D}$ is then continuous with respect to lower cuts if

E6. $\overline{\mathrm{E}}(f)=\lim _{c \rightarrow-\infty} \overline{\mathrm{E}}\left(f^{\vee c}\right)$ for all $f \in \mathscr{D}$.

The limit on the right hand side exists because $f^{\vee c} \in \overline{\mathscr{L}}_{b}(\mathscr{Y})$ is non-decreasing in $c \in \mathbb{R}$ and $\overline{\mathrm{E}}$ satisfies [E4 by assumption. Axiom E6 will play a particularly important role further on, where we will study the compatibility of local and global game-theoretic upper expectations. Moreover, we will also already useE6]in this section, to establish an important relation between our definition of an upper expectation and the one used by Shafer and Vovk.

The definition of Shafer and Vovk (and Takemura) [13, Section 6.1] only differs from Definition!with regard to the choice of domain and the variables for which axiomsE2]E3]andE4]should hold. Their definition is stated for $\mathscr{D}=\overline{\mathscr{L}}(\mathscr{Y})$ and requires that E2 E4 should hold on all of $\overline{\mathscr{L}}(\mathscr{Y})$ :

E2'. $\overline{\mathrm{E}}(f+g) \leq \overline{\mathrm{E}}(f)+\overline{\mathrm{E}}(g)$ for all $f, g \in \overline{\mathscr{L}}(\mathscr{Y})$;

E3'. $\overline{\mathrm{E}}(\lambda f)=\lambda \overline{\mathrm{E}}(f)$ for all $\lambda \in \mathbb{R}_{>0}$ and all $f \in \overline{\mathscr{L}}(\mathscr{Y})$;

E4'. $f \leq g \Rightarrow \overline{\mathrm{E}}(f) \leq \overline{\mathrm{E}}(g)$ for all $f, g \in \overline{\mathscr{L}}(\mathscr{Y})$,

where they use the convention that $+\infty-\infty=-\infty+\infty=+\infty$. This is a typical choice when working with upper expectations—also see [4] where they use the dual convention for lower expectations—and we will henceforth use this convention without mentioning it explicitly. So, for example, $a \geq b$ implies that $a-b \geq 0$, but not necessarily $0 \geq b-a$ for any two $a$ and $b$ in $\overline{\mathbb{R}}$. Moreover, we also adopt the conventions that $c-\infty=-\infty$ for all real $c,-\infty-\infty=-\infty, \lambda(-\infty)=-\infty$ and $(-\lambda)(+\infty)=-\infty$ for all $\lambda \in \overline{\mathbb{R}}_{>0}$ and $0(-\infty)=0$.

Now, note that axioms E2 E4 are weaker than axioms E2' $\mathrm{E} 4$ ' Indeed, any extended real-valued map $\overline{\mathrm{E}}$ on $\mathscr{D}=\overline{\mathscr{L}}(\mathscr{Y})$ that satisfies E2'E4' automatically satisfies E2 E4 simply because $\overline{\mathscr{L}}_{\mathrm{b}}(\mathscr{Y}) \subset \overline{\mathscr{L}}(\mathscr{Y})$. The converse is not necessarily true becauseE2 E4 do not impose any conditions on the values of $\overline{\mathrm{E}}$ on the domain $\overline{\mathscr{L}}(\mathscr{Y}) \backslash \overline{\mathscr{L}}_{\mathrm{b}}(\mathscr{Y})$. Therefore, and since our domain $\mathscr{D}$ is not bound to be equal to $\overline{\mathscr{L}}(\mathscr{Y})$, our definition of an upper expectation is 
more general than Shafer and Vovk's. However, if we choose $\mathscr{D}=\overline{\mathscr{L}}(\mathscr{Y})$ and, besidesE1 E5 additionally impose E6] on our upper expectations, then we always obtain an upper expectation according to Shafer and Vovk:

Proposition 1. Consider any non-empty set $\mathscr{Y}$ and any upper expectation $\overline{\mathrm{E}}$ on $\overline{\mathscr{L}}(\mathscr{Y})$. Then $\overline{\mathrm{E}}$ satisfies $\mathrm{E} 2$ 'E4' if it satisfies[E6,

Proof. E2' Consider any two $f, g \in \overline{\mathscr{L}}(\mathscr{Y})$ and any $c \in \mathbb{R}$. Then, since $f^{\vee c} \geq c$ and $g^{\vee c} \geq c$, we have that $f^{\vee c}+g^{\vee c} \geq 2 c$. In a similar way, we deduce that $f^{\vee c}+g^{\vee c} \geq f+g$. Hence, combining both inequalities, we obtain that $f^{\vee c}+g^{\vee c} \geq \max \{f+g, 2 c\}=(f+g)^{\vee 2 c}$. Moreover note that $f^{\vee c}, g^{\vee c}$ and $(f+g)^{\vee 2 c}$ are all variables in $\overline{\mathscr{L}}_{b}(\mathscr{Y})$, so we can applyE4 and subsequentlyE2 to infer that

$$
\overline{\mathrm{E}}\left((f+g)^{\vee 2 c}\right) \leq \overline{\mathrm{E}}\left(f^{\vee c}+g^{\vee c}\right) \leq \overline{\mathrm{E}}\left(f^{\vee c}\right)+\overline{\mathrm{E}}\left(g^{\vee c}\right) .
$$

The inequality above holds for any $c \in \mathbb{R}$, so we have that

$$
\begin{gathered}
\overline{\mathrm{E}}(f+g) \stackrel{\stackrel{\mathrm{E} 6}{=}}{\lim _{c \rightarrow-\infty}} \overline{\mathrm{E}}\left((f+g)^{\vee c}\right)=\lim _{c \rightarrow-\infty} \overline{\mathrm{E}}\left((f+g)^{\vee 2 c}\right) \leq \lim _{c \rightarrow-\infty}\left[\overline{\mathrm{E}}\left(f^{\vee c}\right)+\overline{\mathrm{E}}\left(g^{\vee c}\right)\right]=\lim _{c \rightarrow-\infty} \overline{\mathrm{E}}\left(f^{\vee c}\right)+\lim _{c \rightarrow-\infty} \overline{\mathrm{E}}\left(g^{\vee c}\right) \\
\stackrel{\mathrm{E} 6}{=} \overline{\mathrm{E}}(f)+\overline{\mathrm{E}}(g),
\end{gathered}
$$

where the existence of all the limits follows from the monotonicity [E4] of $\overline{\mathrm{E}}$, and where the second to last equality follows from the fact that $\overline{\mathrm{E}}\left(f^{\vee c}\right)$ and $\overline{\mathrm{E}}\left(g^{\vee c}\right)$ are non-decreasing in $c$ and our convention that $+\infty-\infty=+\infty$.

E3. Consider any $f \in \overline{\mathscr{L}}(\mathscr{Y})$. First note that, since multiplication with a positive constant $\lambda \in \mathbb{R}_{>0}$ is order preserving on $\overline{\mathbb{R}}$, we have that

$$
\max \{\lambda f(y), c\}=\max \left\{\lambda f(y), \lambda \frac{c}{\lambda}\right\}=\lambda \max \left\{f(y), \frac{c}{\lambda}\right\} \text { for all } y \in \mathscr{Y} \text { and all } c \in \mathbb{R} .
$$

Hence, $(\lambda f)^{\vee c}=\lambda f^{\vee c / \lambda}$ for all $c \in \mathbb{R}$ and all $\lambda \in \mathbb{R}_{>0}$. Since moreover $f^{v^{c / \lambda}} \in \overline{\mathscr{L}}_{b}(\mathscr{Y})$, we can apply[E3 to infer that $\overline{\mathrm{E}}\left((\lambda f)^{\vee c}\right)=\overline{\mathrm{E}}\left(\lambda f^{\vee c / \lambda}\right)=\lambda \overline{\mathrm{E}}\left(f^{\vee c / \lambda}\right)$ for all $c \in \mathbb{R}$ and all $\lambda \in \mathbb{R}_{>0}$. This then implies that

$$
\overline{\mathrm{E}}(\lambda f) \stackrel{\text { 㐭 }}{=} \lim _{c \rightarrow-\infty} \overline{\mathrm{E}}\left((\lambda f)^{\vee c}\right)=\lim _{c \rightarrow-\infty} \lambda \overline{\mathrm{E}}\left(f^{\vee c / \lambda}\right)=\lambda \lim _{c \rightarrow-\infty} \overline{\mathrm{E}}\left(f^{\vee c / \lambda}\right)=\lambda \lim _{c \rightarrow-\infty} \overline{\mathrm{E}}\left(f^{\vee c}\right)^{\stackrel{\mathrm{EG}}{=}} \lambda \overline{\mathrm{E}}(f) \text { for all } \lambda \in \mathbb{R}_{>0} .
$$

E4] This follows trivially from the monotonicity [E4] of $\overline{\mathrm{E}}$ on $\overline{\mathscr{L}}_{b}(\mathscr{Y})$ in combination with E6

The following counterexample shows that the converse is not necessarily true and hence, that axioms E1 E6 define a strictly smaller set of upper expectation operators on $\overline{\mathscr{L}}(\mathscr{Y})$ compared to Shafer and Vovk's axioms.

Example 1. Consider any set $\mathscr{Y}$ such that $|\mathscr{Y}|>1$ and the extended real-valued map $\overline{\mathrm{E}}: \overline{\mathscr{L}}(\mathscr{Y}) \rightarrow \overline{\mathbb{R}}$ defined by

$$
\overline{\mathrm{E}}(f):= \begin{cases}-\infty & \text { if } f<+\infty \text { pointwise and } f(y)=-\infty \text { for some } y \in \mathscr{Y} ; \\ \sup f & \text { otherwise }\end{cases}
$$

for all $f \in \overline{\mathscr{L}}(\mathscr{Y})$. We show that $\overline{\mathrm{E}}$ satisfies $\mathrm{E1}, \mathrm{E2}$ E4] and $\mathrm{E5}$, but not $\mathrm{E} 6$,

E1: This follows trivially from the definition of $\overline{\mathrm{E}}$.

E2'. Consider any two $f, g \in \overline{\mathscr{L}}(\mathscr{Y})$. If there is some $y \in \mathscr{Y}$ such that $f(y)=+\infty$, then we have that $\overline{\mathrm{E}}(f)=+\infty$ and therefore also that $\overline{\mathrm{E}}(f)+\overline{\mathrm{E}}(g)=+\infty$, which implies the desired inequality. Due to symmetry, the inequality is also satisfied if $g(y)=+\infty$ for some $y \in \mathscr{Y}$. Hence, consider the case where both $f<+\infty$ and $g<+\infty$ pointwise. 
Then we clearly also have that $f+g<+\infty$ pointwise. If moreover $f(y)=-\infty$ for some $y \in \mathscr{Y}$, then also $f(y)+$ $g(y)=-\infty$ (because $g(y)<+\infty)$ which, together with the fact that $f+g<+\infty$ pointwise, implies that $\overline{\mathrm{E}}(f+g)=$ $-\infty$ and thus the desired inequality. Once more, the same can be concluded if $g(y)=-\infty$ for some $y \in \mathscr{Y}$ because of symmetry. Hence, we are left with the situation where both $f$ and $g$-and therefore also $f+g$-are real-valued. Then we can immediately infer that $\overline{\mathrm{E}}(f+g)=\sup (f+g) \leq \sup f+\sup g=\overline{\mathrm{E}}(f)+\overline{\mathrm{E}}(g)$.

E3'. Consider any $\lambda \in \mathbb{R}_{>0}$ and any $f \in \bar{L}(\mathscr{Y})$. If $f<+\infty$ pointwise and $f(y)=-\infty$ for some $y \in \mathscr{Y}$, then also $\lambda f<+\infty$ pointwise and $\lambda f(y)=-\infty$, which implies that $\lambda \overline{\mathrm{E}}(f)=\lambda(-\infty)=-\infty=\overline{\mathrm{E}}(\lambda f)$. Otherwise, if $f>-\infty$ pointwise or $f(y)=+\infty$ for some $y \in \mathscr{Y}$, then also $\lambda f>-\infty$ pointwise or $\lambda f(y)=+\infty$, which implies that $\lambda \overline{\mathrm{E}}(f)=\lambda \sup f=\sup \lambda f=\overline{\mathrm{E}}(\lambda f)$.

E4' Consider any $f, g \in \overline{\mathscr{L}}(\mathscr{Y})$ such that $f \leq g$. If $f<+\infty$ pointwise and $f(y)=-\infty$ for some $y \in \mathscr{Y}$, then $\overline{\mathrm{E}}(f)=-\infty$ and therefore automatically $\overline{\mathrm{E}}(f) \leq \overline{\mathrm{E}}(g)$. Otherwise, if $f>-\infty$ pointwise or $f(y)=+\infty$ for some $y \in \mathscr{Y}$, then also $g>-\infty$ pointwise or $g(y)=+\infty$ for some $y \in \mathscr{Y}$. Then it follows from the definition of $\overline{\mathrm{E}}$ that $\overline{\mathrm{E}}(f)=\sup f \leq \sup g=\overline{\mathrm{E}}(g)$.

E5: Consider any non-decreasing sequence $\left\{f_{n}\right\}_{n \in \mathbb{N}_{0}}$ of non-negative variables in $\overline{\mathscr{L}}(\mathscr{Y})$. Since $f_{n} \geq 0>-\infty$ pointwise, we have that $\overline{\mathrm{E}}\left(f_{n}\right)=\sup f_{n}$ for all $n \in \mathbb{N}_{0}$. Clearly, $f:=\lim _{n \rightarrow+\infty} f_{n}$ is non-negative too, so we also have that $\overline{\mathrm{E}}(f)=\sup f$. Hence, we infer that

$$
\lim _{n \rightarrow+\infty} \overline{\mathrm{E}}\left(f_{n}\right)=\lim _{n \rightarrow+\infty} \sup _{y \in \mathscr{Y}} f_{n}(y)=\sup _{n \in \mathbb{N}_{0}} \sup _{y \in \mathscr{Y}} f_{n}(y)=\sup _{y \in \mathscr{Y}} \sup _{n \in \mathbb{N}_{0}} f_{n}(y)=\sup _{y \in \mathscr{Y}} f(y)=\overline{\mathrm{E}}(f),
$$

where the second and the fourth equality follows from the non-decreasing character of $\left\{f_{n}\right\}_{n \in \mathbb{N}_{0}}$.

So $\overline{\mathrm{E}}$ is an upper expectation on $\overline{\mathscr{L}}(\mathscr{Y})$ that moreover satisfies the additional axioms E2' E4' However, it is easy to see that it does not satisfy [E6, Indeed, consider the extended real variable $-\infty \mathbb{I}_{y}$ where $y \in \mathscr{Y}$. Then we have that $\overline{\mathrm{E}}\left(-\infty \mathbb{I}_{y}\right)=-\infty$. On the other hand, $\overline{\mathrm{E}}\left(\left(-\infty \mathbb{I}_{y}\right)^{\vee c}\right)=\overline{\mathrm{E}}\left(c \mathbb{I}_{y}\right)=0$ for all non-positive $c \in \mathbb{R}$ (indeed, note that $\sup c \mathbb{I}_{y}=0$ because $\left.|\mathscr{Y}|>1\right)$. So $\overline{\mathrm{E}}\left(-\infty \mathbb{I}_{y}\right)=+\infty \neq 0=\lim _{c \rightarrow-\infty} \overline{\mathrm{E}}\left(\left(-\infty \mathbb{I}_{y}\right)^{\vee c}\right)$, which implies that $\overline{\mathrm{E}}$ does not satisfyE6

Now, our reason for establishing Proposition—is not merely because of the result itself, but rather because it will allow us to draw the following essential conclusion: as far as the global game-theoretic upper expectationour main object of interest in this paper-is concerned, it does not matter what $\mathscr{D}$ is and whether we do, or do not additionally impose E2' E4' on the local upper expectations. In order to see this, it suffices for now to know that the global game-theoretic upper expectation —which will only be introduced later on in Section 3 —will only depend on the values of our local upper expectations on the (sub)domain $\overline{\mathscr{L}}_{b}(\mathscr{Y})$ (these local upper expectations are simply upper expectations on $\mathscr{D}$, with $\overline{\mathscr{L}}_{b}(\mathscr{Y}) \subseteq \mathscr{D} \subseteq \overline{\mathscr{L}}(\mathscr{Y})$, where $\mathscr{Y}$ is the local state space $\mathscr{X}$ of the considered process). So our statement follows if we can show that letting $\mathscr{D}=\overline{\mathscr{L}}(\mathscr{Y})$ and imposing axioms E2'E4' on (local) upper expectations, does not restrict the possible values that these upper expectations can take on the subdomain $\overline{\mathscr{L}}_{b}(\mathscr{Y})$. To show this, we consider for any upper expectation $\overline{\mathrm{E}}$ on $\mathscr{D}$, with $\overline{\mathscr{L}}_{b}(\mathscr{Y}) \subseteq \mathscr{D} \subseteq \overline{\mathscr{L}}(\mathscr{Y})$, the map $\overline{\mathrm{E}}_{\mathrm{E} 6}: \overline{\mathscr{L}}(\mathscr{Y}) \rightarrow \overline{\mathbb{R}}$ defined by

$$
\overline{\mathrm{E}}_{\mathrm{E} \sigma}(f):=\lim _{c \rightarrow-\infty} \overline{\mathrm{E}}\left(f^{\vee c}\right) \text { for all } f \in \overline{\mathscr{L}}(\mathscr{Y}),
$$

where the right hand side is well-defined because $f^{\vee c} \in \overline{\mathscr{L}}_{b}(\mathscr{Y})$ for any $c \in \mathbb{R}$ and $\overline{\mathrm{E}}$ satisfies E4 (therefore implying the existence of the limit). Then it is quite easy to see that $\overline{\mathrm{E}}_{\mathrm{E} 6}$ coincides with $\overline{\mathrm{E}}$ on $\overline{\mathscr{L}}_{b}(\mathscr{Y})$ and that it is an 
upper expectation that furthermore satisfies E6 and therefore, by Proposition 1 also satisfies E2' E4'

Proposition 2. Consider any non-empty set $\mathscr{Y}$ and any $\overline{\mathscr{L}}_{b}(\mathscr{Y}) \subseteq \mathscr{D} \subseteq \bar{L}(\mathscr{Y})$. For any upper expectation $\overline{\mathrm{E}}$ on $\mathscr{D}$, the associated map $\overline{\mathrm{E}}_{\mathrm{E} 6}$ is an upper expectation on $\overline{\mathscr{L}}(\mathscr{Y})$ that coincides with $\overline{\mathrm{E}}$ on $\overline{\mathscr{L}}_{b}(\mathscr{Y})$-and hence extends $\overline{\mathrm{E}}$ if $\mathscr{D}=\overline{\mathscr{L}}_{b}(\mathscr{Y})$ - and that moreover satisfies [E6 and E2' E4'

Proof. The map $\overline{\mathrm{E}}_{\mathrm{E} 6}$ coincides with $\overline{\mathrm{E}}$ on $\overline{\mathscr{L}}_{b}(\mathscr{Y})$-and hence extends $\overline{\mathrm{E}}$ if $\mathscr{D}=\overline{\mathscr{L}}_{b}(\mathscr{Y})$-because, for any $f \in$ $\overline{\mathscr{L}}_{b}(\mathscr{Y})$, we have that $f^{\vee c}=f$ for all $c \in \mathbb{R}$ such that $c \leq \inf f$, and hence, $\overline{\mathrm{E}}_{\mathrm{E} 6}(f)=\lim _{c \rightarrow-\infty} \overline{\mathrm{E}}\left(f^{\vee c}\right)=\overline{\mathrm{E}}(f)$. Since $\overline{\mathrm{E}}_{\mathrm{E} 6}$ coincides with $\overline{\mathrm{E}}$ on $\overline{\mathscr{L}}_{b}(\mathscr{Y})$ and since $\overline{\mathrm{E}}$ is an upper expectation, it is now immediate that $\overline{\mathrm{E}}_{\mathrm{E} 6}$ is an upper expectation as well. Furthermore, again since $\overline{\mathrm{E}}_{\mathrm{E} 6}$ coincides with $\overline{\mathrm{E}}$ on $\overline{\mathscr{L}}_{b}(\mathscr{Y})$, it follows from its definition that $\overline{\mathrm{E}}_{\mathrm{E} 6}$ satisfies[E6 and hence, due to Proposition 1, that it also satisfies E2' E4'

This result, together with the fact that our global game-theoretic upper expectations will only depend on the restriction of our local models to $\overline{\mathscr{L}}_{b}(\mathscr{Y})$, indeed confirms our statement above. For this reason, and since letting $\mathscr{D}=\overline{\mathscr{L}}(\mathscr{Y})$ and imposing axioms E2' $\mathrm{E4}^{\prime}$ would only reduce the generality of what we do, we have chosen to only require that $\overline{\mathscr{L}}_{b}(\mathscr{Y}) \subseteq \mathscr{D} \subseteq \overline{\mathscr{L}}(\mathscr{Y})$ and to adoptE1,E5 as our axioms for an upper expectation. In some cases, however, when we desire compatibility of local and global models, we will let $\mathscr{D}=\overline{\mathscr{L}}(\mathscr{Y})$ and impose E6 on our local upper expectations, which by Proposition 1 and Example 1 is actually stronger than imposingE2'E4'

\subsection{Some Basic Properties of Upper Expectations}

We now continue this section with a second part in which we establish various properties of upper expectations that will prove convenient for the rest of the paper, or, as is for example the case for Proposition 5 below, are just interesting in their own right.

Proposition 3. Consider any non-empty set $\mathscr{Y}$, any $\overline{\mathscr{L}}_{b}(\mathscr{Y}) \subseteq \mathscr{D} \subseteq \overline{\mathscr{L}}(\mathscr{Y})$ and any extended real-valued map $\overline{\mathrm{E}}$ on $\mathscr{D}$ that satisfies E1 E4 and let $\underline{\mathrm{E}}$ be the conjugate map of $\overline{\mathrm{E}}$ defined on $-\mathscr{D}$. Then we have that

E7. $-\infty<\inf f \leq \overline{\mathrm{E}}(f) \leq \sup f$ for all $f \in \overline{\mathscr{L}}_{b}(\mathscr{Y})$;

E8. $\overline{\mathrm{E}}(f+\mu)=\overline{\mathrm{E}}(f)+\mu$ for all $\mu \in \mathbb{R} \cup\{+\infty\}$ and all $f \in \overline{\mathscr{L}}_{b}(\mathscr{Y})$;

E9. $\overline{\mathrm{E}}(\lambda f)=\lambda \overline{\mathrm{E}}(f)$ for all $\lambda \in \mathbb{R}_{\geq 0}$ and all $f \in \overline{\mathscr{L}}_{b}(\mathscr{Y})$.

E10. $\underline{\mathrm{E}}(f+g) \leq \overline{\mathrm{E}}(f)+\underline{\mathrm{E}}(g) \leq \overline{\mathrm{E}}(f+g)$ for all $f, g \in \mathscr{L}(\mathscr{Y})$;

E11. if $\lim _{n \rightarrow+\infty} \sup \left|f-f_{n}\right|=0$ then $\lim _{n \rightarrow+\infty}\left|\overline{\mathrm{E}}(f)-\overline{\mathrm{E}}\left(f_{n}\right)\right|=0$ for any sequence $\left\{f_{n}\right\}_{n \in \mathbb{N}_{0}}$ in $\mathscr{L}(\mathscr{Y})$.

If $\overline{\mathrm{E}}$ moreover satisfies $\mathrm{E5}$, then we also have that

E12. $\lim _{n \rightarrow+\infty} \overline{\mathrm{E}}\left(f_{n}\right)=\overline{\mathrm{E}}\left(\lim _{n \rightarrow+\infty} f_{n}\right)$ for any non-decreasing sequence $\left\{f_{n}\right\}_{n \in \mathbb{N}_{0}}$ in $\overline{\mathscr{L}}_{b}(\mathscr{Y})$.

Proof. E7 Consider any $f \in \overline{\mathscr{L}}_{b}(\mathscr{Y})$. If $\sup f=+\infty$, we trivially have that $\overline{\mathrm{E}}(f) \leq \sup f$. If $\sup f$ is real, it follows immediately from E4 that $\overline{\mathrm{E}}(f) \leq \overline{\mathrm{E}}(\sup f)$ and therefore that $\overline{\mathrm{E}}(f) \leq \sup f$ because of E1. That $\sup f=-\infty$, is impossible because $f$ is bounded below. To see that $-\infty<\inf f \leq \overline{\mathrm{E}}(f)$, note that $\inf f$ is real or equal to $+\infty$ [because $f$ is bounded below] and therefore that $-\infty<\inf f$ is automatically satisfied. Moreover, for any real 
$\alpha<\inf f$ we clearly have that $\alpha<f$, implying by E4] andE1] that $\alpha<\overline{\mathrm{E}}(f)$. Since this holds for any $\alpha<\inf f$ we indeed have that inf $f \leq \overline{\mathrm{E}}(f)$.

E8 That $\overline{\mathrm{E}}(f+\mu) \leq \overline{\mathrm{E}}(f)+\mu$ for all real $\mu$ and all $f \in \overline{\mathscr{L}}_{b}(\mathscr{Y})$, follows directly from E2 andE1, The other inequality follows from the fact that

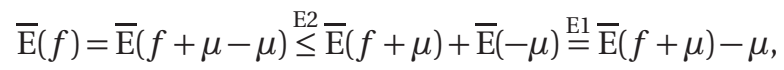

for all $f \in \overline{\mathscr{L}}_{b}(\mathscr{Y})$ and all real $\mu$. If $\mu=+\infty$, then since $\overline{\mathrm{E}}(f)>-\infty$ [because of E7], it remains to check that $\overline{\mathrm{E}}(+\infty)=+\infty$, which follows trivially fromE7,

E9. If $\lambda=0$, we have to show that $\overline{\mathrm{E}}(0)=0$, which follows immediately from E1] Otherwise, if $\lambda \in \mathbb{R}_{>0}$, the equality follows from E3

E10. For all $f, g \in \mathscr{L}(\mathscr{Y})$ we have that

$$
\overline{\mathrm{E}}(f)=\overline{\mathrm{E}}(f+g-g) \stackrel{\mathrm{E} 2}{\leq} \overline{\mathrm{E}}(f+g)+\overline{\mathrm{E}}(-g)=\overline{\mathrm{E}}(f+g)-\underline{\mathrm{E}}(g),
$$

where the last step follows from the definition of $\underline{\mathrm{E}}$. Hence, because $-\underline{\mathrm{E}}(g)=\overline{\mathrm{E}}(-g)$ is real by[E7][ $g$ is a gamble], we have that $\overline{\mathrm{E}}(f)+\underline{\mathrm{E}}(g) \leq \overline{\mathrm{E}}(f+g)$ for all $f, g \in \mathscr{L}(\mathscr{Y})$. The remaining inequality then follows immediately from conjugacy. Indeed, for any $f, g \in \mathscr{L}(\mathscr{Y})$, we already have that $\overline{\mathrm{E}}(-g)+\underline{\mathrm{E}}(-f) \leq \overline{\mathrm{E}}(-g+(-f))$. By conjugacy, this implies that $-\underline{\mathrm{E}}(g)-\overline{\mathrm{E}}(f) \leq-\underline{\mathrm{E}}(g+f)$ and therefore that $\overline{\mathrm{E}}(f)+\underline{\mathrm{E}}(g) \geq \underline{\mathrm{E}}(f+g)$ for any $f, g \in \mathscr{L}(\mathscr{Y})$.

E11\} It is easy to see that, if $\lim _{n \rightarrow+\infty} \sup \left|f-f_{n}\right|=0$ for some sequence $\left\{f_{n}\right\}_{n \in \mathbb{N}_{0}}$ of gambles, then $f$ is also a gamble and so is each $f-f_{n}$. Hence, it follows from E10 that

$$
\underline{\mathrm{E}}\left(f-f_{n}\right) \leq \overline{\mathrm{E}}(f)+\underline{\mathrm{E}}\left(-f_{n}\right) \leq \overline{\mathrm{E}}\left(f-f_{n}\right) \text { for all } n \in \mathbb{N}_{0} .
$$

If we now applyE7 to $\overline{\mathrm{E}}\left(f-f_{n}\right)$, and $\mathrm{E} 7$ and conjugacy to $\underline{\mathrm{E}}\left(f-f_{n}\right)$, it follows from [1] that $\inf \left(f-f_{n}\right) \leq \overline{\mathrm{E}}(f)+$ $\underline{\mathrm{E}}\left(-f_{n}\right) \leq \sup \left(f-f_{n}\right)$ for all $n \in \mathbb{N}_{0}$. Since moreover $\overline{\mathrm{E}}(f)+\underline{\mathrm{E}}\left(-f_{n}\right)=\overline{\mathrm{E}}(f)-\overline{\mathrm{E}}\left(f_{n}\right)$ for all $n \in \mathbb{N}_{0}$, we then have that $\lim _{n \rightarrow+\infty}\left|\overline{\mathrm{E}}(f)-\overline{\mathrm{E}}\left(f_{n}\right)\right|=0$ due to the fact that $\lim _{n \rightarrow+\infty} \sup \left|f-f_{n}\right|=0$.

E12. Let $\left\{f_{n}\right\}_{n \in \mathbb{N}_{0}}$ be any non-decreasing sequence in $\overline{\mathscr{L}}_{b}(\mathscr{Y})$. Since $f_{0}$ is bounded below and $\left\{f_{n}\right\}_{n \in \mathbb{N}_{0}}$ is nondecreasing, $\left\{f_{n}\right\}_{n \in \mathbb{N}_{0}}$ is uniformly bounded below by some $M \in \mathbb{R}$. Therefore, $\left\{f_{n}-M\right\}_{n \in \mathbb{N}_{0}}$ is a non-decreasing sequence of non-negative variables in $\overline{\mathscr{L}}(\mathscr{Y})$. Hence, due toE5, we have that $\lim _{n \rightarrow+\infty} \overline{\mathrm{E}}\left(f_{n}-M\right)=\overline{\mathrm{E}}\left(\lim _{n \rightarrow+\infty} f_{n}-\right.$ $M$ ), which is equivalent to $\lim _{n \rightarrow+\infty} \overline{\mathrm{E}}\left(f_{n}\right)=\overline{\mathrm{E}}\left(\lim _{n \rightarrow+\infty} f_{n}\right)$ due to E8] [which is applicable because $\lim _{n \rightarrow+\infty} f_{n}$ and all $f_{n}$ are bounded below].

As a consequence of their continuity with respect to non-decreasing sequences [E5], upper expectations also satisfy the following countable sub-additivity property.

Proposition 4. Consider any non-empty set $\mathscr{Y}$, any $\overline{\mathscr{L}}_{b}(\mathscr{Y}) \subseteq \mathscr{D} \subseteq \overline{\mathscr{L}}(\mathscr{Y})$ and any upper expectation $\overline{\mathrm{E}}$ on $\mathscr{D}$. Then $\overline{\mathrm{E}}\left(\sum_{n \in \mathbb{N}_{0}} f_{n}\right) \leq \sum_{n \in \mathbb{N}_{0}} \overline{\mathrm{E}}\left(f_{n}\right)$ for any sequence $\left\{f_{n}\right\}_{n \in \mathbb{N}_{0}}$ of non-negative variables in $\overline{\mathscr{L}}_{b}(\mathscr{Y})$.

Proof. Consider the sequence $\left\{g_{n}\right\}_{n \in \mathbb{N}_{0}}$ of non-negative variables defined by $g_{n}:=\sum_{i=0}^{n} f_{i}$ for all $n \in \mathbb{N}_{0}$. Then, $\left\{g_{n}\right\}_{n \in \mathbb{N}_{0}}$ is non-decreasing because $\left\{f_{n}\right\}_{n \in \mathbb{N}_{0}}$ is non-negative. Moreover, it is clear that $\left\{g_{n}\right\}_{n \in \mathbb{N}_{0}}$ converges pointwise to $\sum_{n \in \mathbb{N}_{0}} f_{n}$. Hence, we can applyE5 to find that

$$
\overline{\mathrm{E}}\left(\sum_{n \in \mathbb{N}_{0}} f_{n}\right)=\lim _{n \rightarrow+\infty} \overline{\mathrm{E}}\left(g_{n}\right)=\lim _{n \rightarrow+\infty} \overline{\mathrm{E}}\left(\sum_{i=0}^{n} f_{i}\right) \stackrel{\mathrm{E} 2}{\leq} \lim _{n \rightarrow+\infty} \sum_{i=0}^{n} \overline{\mathrm{E}}\left(f_{i}\right)=\sum_{n \in \mathbb{N}_{0}} \overline{\mathrm{E}}\left(f_{n}\right),
$$


where the limit on the right hand side of the inequality exists because all $\overline{\mathrm{E}}\left(f_{n}\right)$ are non-negative as a consequence of E7

Finally, the following proposition shows that for a finite set $\mathscr{Y}$, the continuity axiom[E5 can in fact be replaced by a much simpler property.

Proposition 5. For any finite non-empty set $\mathscr{Y}$ and any $\overline{\mathscr{L}}_{b}(\mathscr{Y}) \subseteq \mathscr{D} \subseteq \overline{\mathscr{L}}(\mathscr{Y})$, an extended real-valued map $\overline{\mathrm{E}}$ on $\mathscr{D}$ is an upper expectation if and only if it satisfies E1 E4 and

E13. $(+\infty) \overline{\mathrm{E}}(f)=\overline{\mathrm{E}}((+\infty) f)$ for all non-negative $f \in \overline{\mathscr{L}}_{b}(\mathscr{Y})$.

Proof. We first prove the direct implication; that is, we consider any upper expectation $\overline{\mathrm{E}}$ on $\mathscr{D}$ and show that it satisfiesE13. Fix any non-negative $f \in \overline{\mathscr{L}}_{b}(\mathscr{Y})$ and observe that $\{n f\}_{n \in \mathbb{N}}$ is a non-decreasing sequence in $\overline{\mathscr{L}}(\mathscr{Y})$ that converges pointwise to $(+\infty) f$ [because of the convention that $(+\infty) 0=0$ ]. Hence,

$$
\overline{\mathrm{E}}((+\infty) f)=\overline{\mathrm{E}}\left(\lim _{n \rightarrow+\infty} n f\right) \stackrel{\mathrm{E12}}{\stackrel{\underline{\mathrm{E}}}{=}} \lim _{n \rightarrow+\infty} \overline{\mathrm{E}}(n f) \stackrel{\mathrm{E} 3}{=} \lim _{n \rightarrow+\infty} n \overline{\mathrm{E}}(f)=(+\infty) \overline{\mathrm{E}}(f),
$$

where we once more used the convention that $(+\infty) 0=0$ for the last step, together with the fact that $\overline{\mathrm{E}}(f) \geq 0$ because of E7

To prove the converse implication, consider any extended real-valued map $\overline{\mathrm{E}}$ on $\mathscr{D}$ that satisfies E1-E4 and E13 Let $\left\{f_{n}\right\}_{n \in \mathbb{N}_{0}}$ be a non-decreasing sequence of non-negative variables in $\overline{\mathscr{L}}_{b}(\mathscr{Y})$ and let $f:=\lim _{n \rightarrow+\infty} f_{n}$. We show that $\lim _{n \rightarrow+\infty} \overline{\mathrm{E}}\left(f_{n}\right)=\overline{\mathrm{E}}(f)$. Because $\left\{f_{n}\right\}_{n \in \mathbb{N}_{0}}$ is non-decreasing, we have that $f_{n} \leq f_{n+1} \leq f$ for all $n \in \mathbb{N}_{0}$. Then it follows from E4][which we are allowed to use because $f$ and all $f_{n}$ are non-negative and therefore bounded below] that $\overline{\mathrm{E}}\left(f_{n}\right) \leq \overline{\mathrm{E}}\left(f_{n+1}\right) \leq \overline{\mathrm{E}}(f)$ for all $n \in \mathbb{N}_{0}$. Hence, $\lim _{n \rightarrow+\infty} \overline{\mathrm{E}}\left(f_{n}\right)$ exists and $\lim _{n \rightarrow+\infty} \overline{\mathrm{E}}\left(f_{n}\right) \leq \overline{\mathrm{E}}(f)$. To show that the converse inequality holds, let $A:=\{y \in \mathscr{Y}: f(y)=+\infty\}$ and consider the following two cases.

If $\overline{\mathrm{E}}\left(\mathbb{I}_{A}\right)=0$, we have that

$$
\overline{\mathrm{E}}(f)=\overline{\mathrm{E}}\left((+\infty) \mathbb{I}_{A}+f \mathbb{I}_{A^{c}}\right) \stackrel{\mathrm{E} 2}{\leq} \overline{\mathrm{E}}\left((+\infty) \mathbb{I}_{A}\right)+\overline{\mathrm{E}}\left(f \mathbb{I}_{A^{c}}\right) \stackrel{\underline{\mathrm{E} 13}}{=}(+\infty) \overline{\mathrm{E}}\left(\mathbb{I}_{A}\right)+\overline{\mathrm{E}}\left(f \mathbb{I}_{A^{c}}\right)=\overline{\mathrm{E}}\left(f \mathbb{I}_{A^{c}}\right)
$$

Because $f \mathbb{I}_{A^{c}}$ is real-valued [it cannot be $-\infty$ because it is non-negative] and $\mathscr{Y}$ is finite, $f \mathbb{I}_{A^{c}}$ is a gamble and $\left\{f_{n} \mathbb{I}_{A^{c}}\right\}_{n \in \mathbb{N}_{0}}$ converges uniformly to $f \mathbb{I}_{A^{c}} .\left\{f_{n} \mathbb{I}_{A^{c}}\right\}_{n \in \mathbb{N}_{0}}$ is moreover also a sequence of gambles because its elements are non-negative and are bounded from above by the gamble $f \mathbb{I}_{A^{c}}$. Hence, it follows fromE11 that

$$
\overline{\mathrm{E}}\left(f \mathbb{I}_{A^{c}}\right)=\lim _{n \rightarrow+\infty} \overline{\mathrm{E}}\left(f_{n} \mathbb{I}_{A^{c}}\right) \leq \lim _{n \rightarrow+\infty} \overline{\mathrm{E}}\left(f_{n}\right),
$$

where we used the non-negativity of $\left\{f_{n}\right\}_{n \in \mathbb{N}_{0}}$ andE4 in the last step. Together with Equation [2), this then leads to the desired inequality.

If $\overline{\mathrm{E}}\left(\mathbb{I}_{A}\right) \neq 0$, we have that $\overline{\mathrm{E}}\left(\mathbb{I}_{A}\right)>0$ because of $\mathrm{E} 7$ Furthermore, all $f_{n}$ are non-negative, and therefore

$$
\overline{\mathrm{E}}\left(f_{n}\right) \stackrel{\text { E4 }}{\geq} \overline{\mathrm{E}}\left(f_{n} \mathbb{I}_{A}\right) \stackrel{\text { E4 }}{\geq} \overline{\mathrm{E}}\left(\left[\inf _{y \in A} f_{n}(y)\right] \mathbb{I}_{A}\right) \stackrel{\mathrm{Eg} \mathrm{E} 13}{=}\left[i_{y \in A} f_{n}(y)\right] \overline{\mathrm{E}}\left(\mathbb{I}_{A}\right) \text { for all } n \in \mathbb{N}_{0}
$$

Since $\left\{f_{n}\right\}_{n \in \mathbb{N}_{0}}$ converges to $+\infty$ on $A$ and $A$ is moreover finite [since $\mathscr{Y}$ is], we have that $\lim _{n \rightarrow+\infty} \inf _{y \in A} f_{n}(y)=$ $+\infty$. This implies, together with $\overline{\mathrm{E}}\left(\mathbb{I}_{A}\right)>0$ and 3), that $\lim _{n \rightarrow+\infty} \overline{\mathrm{E}}\left(f_{n}\right)=+\infty$. Hence, the desired inequality follows. 


\subsection{An Alternative Characterisation using Coherence}

The axioms in Definition] are rather abstract, particularly because the concept of infinity plays such a prominent role in them. Walley [24] avoids this issue by only considering upper expectations-which he calls upper previsions-on gambles, that is, bounded real-valued variables. This allows him to give upper expectations a clear behavioural interpretation in terms of attitudes towards gambling [11, 24]. Concretely, the upper expectation $\overline{\mathrm{E}}(f)$ for any $f \in \mathscr{L}(\mathscr{Y})$ then represents a subject's infimum selling price for the gamble $f$. This interpretation in turn leads to a notion of rationality that he calls coherence.

Definition 2. Consider any non-empty set $\mathscr{Y}$ and any map $\overline{\mathrm{E}}$ on the linear space $\mathscr{L}(\mathscr{Y})$ of all gambles on $\mathscr{Y}$. Then $\overline{\mathrm{E}}$ is called $a$ coherent upper prevision if it is real-valued and satisfies the following three coherence axioms [24, Definition 2.3.3]:

C1. $\overline{\mathrm{E}}(f) \leq \sup f$ for all $f \in \mathscr{L}(\mathscr{Y})$;

C2. $\overline{\mathrm{E}}(f+g) \leq \overline{\mathrm{E}}(f)+\overline{\mathrm{E}}(g)$ for all $f, g \in \mathscr{L}(\mathscr{Y})$;

C3. $\overline{\mathrm{E}}(\lambda f)=\lambda \overline{\mathrm{E}}(f)$ for all $\lambda \in \mathbb{R}_{>0}$ and $f \in \mathscr{L}(\mathscr{Y})$.

One can easily show [24, Section 2.6.1] that the coherence axioms $\mathrm{Cl}+\mathrm{C} 3 \mathrm{imply}$ the following additional properties, with $\underline{\mathrm{E}}(f):=-\overline{\mathrm{E}}(-f)$ for all $f \in \mathscr{L}(\mathscr{Y})$ :

C4. $f \leq g \Rightarrow \overline{\mathrm{E}}(f) \leq \overline{\mathrm{E}}(g)$ for all $f, g \in \mathscr{L}(\mathscr{Y})$;

C5. $\inf f \leq \underline{\mathrm{E}}(f) \leq \overline{\mathrm{E}}(f) \leq \sup f$ for all $f \in \mathscr{L}(\mathscr{Y})$;

C6. $\overline{\mathrm{E}}(f+\mu)=\overline{\mathrm{E}}(f)+\mu$ for all real $\mu$ and all $f \in \mathscr{L}(\mathscr{Y})$;

C7. $\lim _{n \rightarrow+\infty} \sup \left|f-f_{n}\right|=0 \Rightarrow \lim _{n \rightarrow+\infty}\left|\overline{\mathrm{E}}(f)-\overline{\mathrm{E}}\left(f_{n}\right)\right|=0$ for any sequence $\left\{f_{n}\right\}_{n \in \mathbb{N}_{0}}$ in $\mathscr{L}(\mathscr{Y})$.

Now, in order to use coherent upper previsions as local uncertainty models in a game-theoretic framework, we need to extend their domain to at least the set $\overline{\mathscr{L}}_{b}(\mathscr{Y})$ of all bounded below extended real-valued variables, and in some cases to all of $\overline{\mathscr{L}}(\mathscr{Y})$. We propose the following step-wise approach.

Consider any non-empty set $\mathscr{Y}$ and any $\mathscr{L}(\mathscr{Y}) \subseteq \mathscr{D} \subseteq \overline{\mathscr{L}}(\mathscr{Y})$. Furthermore, for any $f \in \overline{\mathscr{L}}(\mathscr{Y})$ and any $c \in \mathbb{R}$, let $f^{\wedge c}$ be the variable defined by $f^{\wedge c}(y):=\min \{f(y), c\}$ for all $y \in \mathscr{Y}$. Consider now the following continuity property for an extended real-valued map $\overline{\mathrm{E}}$ on $\mathscr{D}$ whose restriction to $\mathscr{L}(\mathscr{Y})$ is a coherent upper prevision:

E14. $\overline{\mathrm{E}}(f)=\lim _{c \rightarrow+\infty} \overline{\mathrm{E}}\left(f^{\wedge c}\right)$ for all $f \in \mathscr{D} \cap \overline{\mathscr{L}}_{b}(\mathscr{Y})$.

Axiom E14 is called 'continuity with respect to upper cuts' (also called 'bounded-above support'). The limit on the right hand side exists, because $f^{\wedge c} \in \mathscr{L}(\mathscr{Y})$ is non-decreasing in $c \in \mathbb{R}$ and $\overline{\mathrm{E}}$-or rather, its restriction to $\mathscr{L}(\mathscr{Y})$-satisfies C4. Similar to what we did with E6 property E14 can be used to (uniquely) extend a coherent upper prevision on $\mathscr{L}(\mathscr{Y})$ to $\overline{\mathscr{L}}_{b}(\mathscr{Y})$. Indeed, let $\overline{\mathrm{E}}$ be any coherent upper prevision on $\mathscr{L}(\mathscr{Y})$ and let $\overline{\mathrm{E}}_{\mathrm{E} 14}: \overline{\mathscr{L}}_{b}(\mathscr{Y}) \rightarrow \overline{\mathbb{R}}$ be defined by

$$
\overline{\mathrm{E}}_{\mathrm{E} 14}(f):=\lim _{c \rightarrow+\infty} \overline{\mathrm{E}}\left(f^{\wedge c}\right) \text { for all } f \in \overline{\mathscr{L}}_{b}(\mathscr{Y}),
$$

where the right hand side is well-defined because $f^{\wedge c} \in \mathscr{L}(\mathscr{Y})$ for all $c \in \mathbb{R}$ and $\overline{\mathrm{E}}$ satisfies $\overline{C 4}$ [which implies the existence of the limit]. Then $\overline{\mathrm{E}}_{\mathrm{E} 14}$ is an extension of $\overline{\mathrm{E}}$ : 
Proposition 6. Consider any non-empty set $\mathscr{Y}$ and let $\overline{\mathrm{E}}$ be any coherent upper prevision on $\mathscr{L}(\mathscr{Y})$. Then $\overline{\mathrm{E}}_{\mathrm{E} 14}$ is an extension of $\overline{\mathrm{E}}$ that satisfies $\mathrm{E} 14$.

Proof. $\overline{\mathrm{E}}_{\mathrm{E} 14}$ is an extension of $\overline{\mathrm{E}}$ because, for any gamble $f \in \mathscr{L}(\mathscr{Y})$, we have that $f^{\wedge c}=f$ for all $c \in \mathbb{R}$ such that $c \geq \sup f$, and hence, $\overline{\mathrm{E}}_{\mathrm{E} 14}(f)=\lim _{c \rightarrow+\infty} \overline{\mathrm{E}}\left(f^{\wedge c}\right)=\overline{\mathrm{E}}(f)$. That $\overline{\mathrm{E}}_{\mathrm{E} 14}$ satisfies E14 now follows immediately from its definition.

Our next result shows, for finite $\mathscr{Y}$, that this extension $\overline{\mathrm{E}}_{\mathrm{E} 14}$ is an upper expectation on $\overline{\mathscr{L}}_{b}(\mathscr{Y})$ and, moreover, that the restriction to $\overline{\mathscr{L}}_{b}(\mathscr{Y})$ of any upper expectation is the extension $\overline{\mathrm{E}}_{\mathrm{E} 14}$ of some coherent upper prevision $\overline{\mathrm{E}}$ on $\mathscr{L}(\mathscr{Y})$.

Proposition 7. Consider any finite non-empty set $\mathscr{Y}$ and any extended real-valued map $\overline{\mathrm{E}}^{\prime}$ on $\mathscr{D}$, with $\overline{\mathscr{L}}_{b}(\mathscr{Y}) \subseteq$ $\mathscr{D} \subseteq \overline{\mathscr{L}}(\mathscr{Y})$. Then $\overline{\mathrm{E}}^{\prime}$ is an upper expectation if and only if there is some coherent upper prevision $\overline{\mathrm{E}}$ on $\mathscr{L}(\mathscr{Y})$ such that $\overline{\mathrm{E}}_{\mathrm{E} 14}$ coincides with $\overline{\mathrm{E}}^{\prime}$ on $\overline{\mathscr{L}}_{b}(\mathscr{Y})$.

The proof of this result is based on the following two lemmas.

Lemma 8. Consider any finite non-empty set $\mathscr{Y}$, any $\overline{\mathscr{L}}_{b}(\mathscr{Y}) \subseteq \mathscr{D} \subseteq \bar{L}(\mathscr{Y})$ and any extended real-valued map $\overline{\mathrm{E}}$ on $\mathscr{D}$ whose restriction to $\mathscr{L}(\mathscr{Y})$ is a coherent upper prevision. Then $\overline{\mathrm{E}}$ satisfies E12 if and only if it satisfies $\mathrm{E} 14$.

Proof. In order to prove the direct implication, suppose that $\overline{\mathrm{E}}$ satisfies E12 and consider any $f \in \overline{\mathscr{L}}_{b}(\mathscr{Y})=\mathscr{D} \cap$ $\overline{\mathscr{L}}_{b}(\mathscr{Y})$ and any non-decreasing sequence $\left\{c_{n}\right\}_{n \in \mathbb{N}_{0}}$ of reals such that $\lim _{n \rightarrow+\infty} c_{n}=+\infty$. Then clearly $\left\{f^{\wedge c_{n}}\right\}_{n \in \mathbb{N}_{0}}$ is non-decreasing in $\overline{\mathscr{L}}_{b}(\mathscr{Y})$ and converges to $f$. Hence, E12 implies that $\lim _{n \rightarrow+\infty} \overline{\mathrm{E}}\left(f^{\wedge c_{n}}\right)=\overline{\mathrm{E}}(f)$. Furthermore, for any $n, m \in \mathbb{N}_{0}$ such that $m>n$, we clearly have that $f^{\wedge c_{n}} \leq f^{\wedge c} \leq f^{\wedge c_{m}}$ for all $c \in \mathbb{R}$ such that $c_{n} \leq c \leq c_{m}$. Due to $\mathrm{C} 4$ [ which we can apply because the restriction of $\overline{\mathrm{E}}$ to $\mathscr{L}(\mathscr{Y})$ is a coherent upper prevision and because $f^{\wedge c}$ is a gamble for all $c \in \mathbb{R}]$, this also implies that $\overline{\mathrm{E}}\left(f^{\wedge c_{n}}\right) \leq \overline{\mathrm{E}}\left(f^{\wedge c}\right) \leq \overline{\mathrm{E}}\left(f^{\wedge c_{m}}\right)$ for all $c \in \mathbb{R}$ such that $c_{n} \leq c \leq c_{m}$. Since this holds for any $n, m \in \mathbb{N}_{0}$ such that $m>n$, it follows that $\lim _{c \rightarrow+\infty} \overline{\mathrm{E}}\left(f^{\wedge c}\right)=\lim _{n \rightarrow+\infty} \overline{\mathrm{E}}\left(f^{\wedge c_{n}}\right)=\overline{\mathrm{E}}(f)$, where the last equality follows from our earlier considerations.

To see that the converse implication holds, suppose that $\overline{\mathrm{E}}$ satisfies E14 and fix any non-decreasing sequence $\left\{f_{n}\right\}_{n \in \mathbb{N}_{0}}$ in $\overline{\mathscr{L}}_{b}(\mathscr{Y})$. Let $f:=\lim _{n \rightarrow+\infty} f_{n} \in \overline{\mathscr{L}}_{b}(\mathscr{Y})$. Then, for any $c \in \mathbb{R},\left\{f_{n}^{\wedge c}\right\}_{n \in \mathbb{N}_{0}}$ is a non-decreasing sequence in $\mathscr{L}(\mathscr{Y})$ that clearly converges pointwise to $f^{\wedge c} \in \mathscr{L}(\mathscr{Y})$. Moreover, since $f^{\wedge c}$ is a real-valued function on a finite set $\mathscr{Y}$, the sequence $\left\{f_{n}^{\wedge c}\right\}_{n \in \mathbb{N}_{0}}$ converges uniformly to $f^{\wedge c}$. Hence, we have that

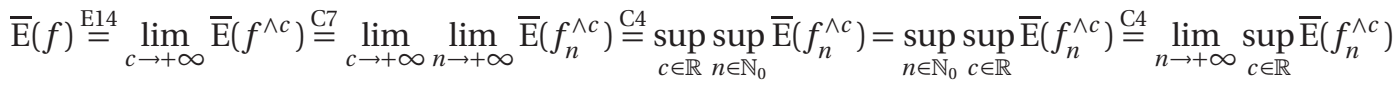

$$
\begin{aligned}
& \stackrel{\text { C4. }}{=} \lim _{n \rightarrow+\infty} \lim _{c \rightarrow+\infty} \overline{\mathrm{E}}\left(f_{n}^{\wedge c}\right) \\
& \stackrel{\text { E14 }}{=} \lim _{n \rightarrow+\infty} \overline{\mathrm{E}}\left(f_{n}\right) \text {. }
\end{aligned}
$$

Lemma 9. Consider any finite non-empty set $\mathscr{Y}$, any $\overline{\mathscr{L}}_{b}(\mathscr{Y}) \subseteq \mathscr{D} \subseteq \overline{\mathscr{L}}(\mathscr{Y})$ and any extended real-valued map $\overline{\mathrm{E}}$ on $\mathscr{D}$. If the restriction of $\overline{\mathrm{E}}$ to $\mathscr{L}(\mathscr{Y})$ is a coherent upper prevision and $\overline{\mathrm{E}}$ satisfies $\mathrm{E} 12$, then $\overline{\mathrm{E}}$ is an upper expectation.

Proof. Assume that the restriction of $\overline{\mathrm{E}}$ to $\mathscr{L}(\mathscr{Y})$ is a coherent upper prevision and $\overline{\mathrm{E}}$ satisfies E12, ThatE1] holds, follows immediately from $\mathrm{C5}$ To prove E2 consider any two $f, g \in \overline{\mathscr{L}}_{b}(\mathscr{Y})$ and the corresponding nondecreasing sequences $\left\{f^{\wedge n}\right\}_{n \in \mathbb{N}_{0}}$ and $\left\{g^{\wedge n}\right\}_{n \in \mathbb{N}_{0}}$ in $\mathscr{L}(\mathscr{Y})$. Then due toE12, $\lim _{n \rightarrow+\infty} \overline{\mathrm{E}}\left(f^{\wedge n}\right)=\overline{\mathrm{E}}(f)$ and $\lim _{n \rightarrow+\infty} \overline{\mathrm{E}}\left(g^{\wedge n}\right)=$ 
$\overline{\mathrm{E}}(g)$. Moreover, $\left\{f^{\wedge n}+g^{\wedge n}\right\}_{n \in \mathbb{N}_{0}}$ is also a non-decreasing sequence in $\mathscr{L}(\mathscr{Y})$ and clearly $\lim _{n \rightarrow+\infty}\left(f^{\wedge n}+g^{\wedge n}\right)=$ $f+g \in \overline{\mathscr{L}}_{b}(\mathscr{Y})$, which again implies byE12 that $\lim _{n \rightarrow+\infty} \overline{\mathrm{E}}\left(f^{\wedge n}+g^{\wedge n}\right)=\overline{\mathrm{E}}(f+g)$. All together, we have that

$$
\overline{\mathrm{E}}(f+g)=\lim _{n \rightarrow+\infty} \overline{\mathrm{E}}\left(f^{\wedge n}+g^{\wedge n}\right) \stackrel{\underline{\mathrm{Cn}}}{\leq} \lim _{n \rightarrow+\infty}\left[\overline{\mathrm{E}}\left(f^{\wedge n}\right)+\overline{\mathrm{E}}\left(g^{\wedge n}\right)\right]=\overline{\mathrm{E}}(f)+\overline{\mathrm{E}}(g),
$$

which concludes the proof of E2,

PropertyE3 can be proved in a similar way. Consider any $\lambda \in \mathbb{R}_{>0}$ and any $f \in \overline{\mathscr{L}}_{b}(\mathscr{Y})$. Note that $\left\{\lambda f^{\wedge n}\right\}_{n \in \mathbb{N}_{0}}$ is a non-decreasing sequence [because $\lambda>0$ ] in $\mathscr{L}(\mathscr{Y})$ that converges pointwise to $\lambda f \in \overline{\mathscr{L}}_{b}(\mathscr{Y})$. Hence,

$$
\overline{\mathrm{E}}(\lambda f)^{\stackrel{\mathrm{E} 12}{=}} \lim _{n \rightarrow+\infty} \overline{\mathrm{E}}\left(\lambda f^{\wedge n}\right)^{\underline{\underline{\underline{C} 3}}} \lim _{n \rightarrow+\infty} \lambda \overline{\mathrm{E}}\left(f^{\wedge n}\right)^{\underline{\mathrm{E} 12}} \lambda \overline{\mathrm{E}}(f) .
$$

To prove E4 consider any two $f, g \in \overline{\mathscr{L}}_{b}(\mathscr{Y})$ such that $f \leq g$, and the corresponding non-decreasing sequences $\left\{f^{\wedge n}\right\}_{n \in \mathbb{N}_{0}}$ and $\left\{g^{\wedge n}\right\}_{n \in \mathbb{N}_{0}}$ in $\mathscr{L}(\mathscr{Y})$. Clearly, $f^{\wedge n} \leq g^{\wedge n}$ for all $n \in \mathbb{N}_{0}$ and therefore $\overline{\mathrm{E}}\left(f^{\wedge n}\right) \leq \overline{\mathrm{E}}\left(g^{\wedge n}\right)$ by $\mathrm{C4}$ Hence, $\lim _{n \rightarrow+\infty} \overline{\mathrm{E}}\left(f^{\wedge n}\right) \leq \lim _{n \rightarrow+\infty} \overline{\mathrm{E}}\left(g^{\wedge n}\right)$ and therefore, because of E12 also $\overline{\mathrm{E}}(f) \leq \overline{\mathrm{E}}(g)$. Finally, propertyE5 follows immediately fromE12

Proof of Proposition7. First assume that $\overline{\mathrm{E}}^{\prime}$ is an upper expectation. We then let $\overline{\mathrm{E}}$ be the restriction of $\overline{\mathrm{E}}^{\prime}$ to $\mathscr{L}(\mathscr{Y}) . \overline{\mathrm{E}}$ trivially satisfies $\mathrm{C} 1 \mathrm{C}$ because $\overline{\mathrm{E}}^{\prime}$ is an upper expectation, and $\overline{\mathrm{E}}$ is real-valued because $\overline{\mathrm{E}}^{\prime}$ satisfies E7. Hence, $\overline{\mathrm{E}}$ is a coherent upper prevision. Moreover, since $\overline{\mathrm{E}}^{\prime}$ satisfies E12 because of Proposition 3 Lemma 8 implies that it also satisfies E14. Hence,

$$
\overline{\mathrm{E}}^{\prime}(f)=\lim _{c \rightarrow+\infty} \overline{\mathrm{E}}^{\prime}\left(f^{\wedge c}\right)=\lim _{c \rightarrow+\infty} \overline{\mathrm{E}}\left(f^{\wedge c}\right)=\overline{\mathrm{E}}_{\mathrm{El} 4}(f) \text { for all } f \in \overline{\mathscr{L}}_{b}(\mathscr{Y}),
$$

where the second step follows from the fact that $\overline{\mathrm{E}}$ is the restriction of $\overline{\mathrm{E}}^{\prime}$ to $\mathscr{L}(\mathscr{Y})$ and because $f^{\wedge c} \in \mathscr{L}(\mathscr{Y})$ for all $c \in \mathbb{R}$, and where the last step follows from the definition of $\overline{\mathrm{E}}_{\mathrm{E} 14}$.

For the converse implication, we assume that there is some coherent upper prevision $\overline{\mathrm{E}}$ on $\mathscr{L}(\mathscr{Y})$ such that $\overline{\mathrm{E}}_{\mathrm{E} 14}$ coincides with $\overline{\mathrm{E}}^{\prime}$ on $\overline{\mathscr{L}}_{b}(\mathscr{Y})$. Now recall from Proposition 6 that $\overline{\mathrm{E}}_{\mathrm{E} 14}$ is an extension of $\overline{\mathrm{E}}$ that satisfies E14 Since $\overline{\mathrm{E}}_{\mathrm{E} 14}$ coincides with $\overline{\mathrm{E}}^{\prime}$ on $\overline{\mathscr{L}}_{b}(\mathscr{Y})$, this implies that $\overline{\mathrm{E}}^{\prime}$ satisfies E14 and that the restriction of $\overline{\mathrm{E}}^{\prime}$ to $\mathscr{L}(\mathscr{Y})$ is equal to $\overline{\mathrm{E}}$ and therefore a coherent upper prevision. Then, by Lemma $8 \overline{\mathrm{E}}^{\prime}$ satisfies $\mathrm{E} 12$, which by Lemma 9 implies that $\overline{\mathrm{E}}^{\prime}$ is an upper expectation.

An important consequence of this alternative characterisation for an upper expectation is that the restriction of an upper expectation to $\overline{\mathscr{L}}_{b}(\mathscr{Y})$ is uniquely determined by its values on the domain $\mathscr{L}(\mathscr{Y})$ of all gambles. This allows us to justify the use of upper expectations on $\overline{\mathscr{L}}_{b}(\mathscr{Y})$-and therefore the use of these as local uncertainty models further on-from a more conventional imprecise probabilities point of view, by combining coherence on gambles withE14 19]. The same cannot be said about the values of our upper expectations on $\overline{\mathscr{L}}(\mathscr{Y}) \backslash \overline{\mathscr{L}}_{b}(\mathscr{Y})$. If we also want these values to be uniquely determined by the values on the gambles, we can additionally impose E6. This is made explicit in our next result, where we will use, for any coherent upper prevision $\overline{\mathrm{E}}$ on $\mathscr{L}(\mathscr{Y})$, the notation $\overline{\mathrm{E}}_{\mathrm{ext}}$ to denote the map $\left[\overline{\mathrm{E}}_{\mathrm{E} 14}\right]_{\mathrm{E} 6}$ that extends $\overline{\mathrm{E}}$ to $\overline{\mathscr{L}}_{b}(\mathscr{Y})$ using E14 and subsequently to $\overline{\mathscr{L}}(\mathscr{Y})$ using E6 Note that $\overline{\mathrm{E}}_{\text {ext }}$ is well-defined because $\overline{\mathrm{E}}_{\mathrm{E} 14}$ is an upper expectation according to Proposition 7 It should moreover be clear that $\overline{\mathrm{E}}_{\text {ext }}$ is also an extension of $\overline{\mathrm{E}}$; this can be checked using Propositions 6 , 7 and 2 
Proposition 10. Consider any finite non-empty set $\mathscr{Y}$, any extended real-valued map $\overline{\mathrm{E}}^{\prime}$ on $\overline{\mathscr{L}}(\mathscr{Y})$ and let $\overline{\mathrm{E}}$ be the restriction of $\overline{\mathrm{E}}^{\prime}$ to $\mathscr{L}(\mathscr{Y})$. Then the following are equivalent:

i. $\overline{\mathrm{E}}^{\prime}$ is an upper expectation on $\overline{\mathscr{L}}(\mathscr{Y})$ that satisfies $\mathrm{E} 6$,

ii. $\overline{\mathrm{E}}$ is a coherent upper prevision such that $\overline{\mathrm{E}}^{\prime}=\overline{\mathrm{E}}_{\mathrm{ext}}$.

iii. $\overline{\mathrm{E}}$ is a coherent upper prevision and $\overline{\mathrm{E}}^{\prime}$ satisfies E6 and E14,

Proof. [i] $\Rightarrow$ iil Assume that $\overline{\mathrm{E}}^{\prime}$ is an upper expectation on $\overline{\mathscr{L}}(\mathscr{Y})$ that satisfies E6, Then, according to Proposition7 there is a coherent upper prevision $\overline{\mathrm{E}}^{*}$ on $\mathscr{L}(\mathscr{Y})$ such that $\overline{\mathrm{E}}^{\prime}(f)=\overline{\mathrm{E}}_{\mathrm{E} 14}^{*}(f)$ for all $f \in \overline{\mathscr{L}}_{b}(\mathscr{Y})$. Then, since $\overline{\mathrm{E}}_{\mathrm{E} 14}^{*}$ is an extension of $\overline{\mathrm{E}}^{*}$ [due to Proposition $\left[\right.$ and since $\overline{\mathrm{E}}^{\prime}$ is an extension of $\overline{\mathrm{E}}$, we have that $\overline{\mathrm{E}}^{*}=\overline{\mathrm{E}}$. On the on hand, this already implies that $\overline{\mathrm{E}}$ is indeed a coherent upper prevision. On the other hand, it implies that $\overline{\mathrm{E}}^{\prime}(f)=\overline{\mathrm{E}}_{\mathrm{E} 14}^{*}(f)=$ $\overline{\mathrm{E}}_{\mathrm{E} 14}(f)$ for all $f \in \overline{\mathscr{L}}_{b}(\mathscr{Y})$. Note that $\overline{\mathrm{E}}_{\mathrm{E} 14}$ is therefore an upper expectation on $\overline{\mathscr{L}}_{b}(\mathscr{Y})$ [because $\overline{\mathrm{E}}^{\prime}$ was assumed to be an upper expectation on $\overline{\mathscr{L}}(\mathscr{Y})]$, which, due to Proposition2 implies that $\overline{\mathrm{E}}_{\mathrm{ext}}$ is an extension of $\overline{\mathrm{E}}_{\mathrm{E} 14}$ satisfying E6. Hence, we have that $\overline{\mathrm{E}}^{\prime}(f)=\overline{\mathrm{E}}_{\mathrm{E} 14}(f)=\overline{\mathrm{E}}_{\mathrm{ext}}(f)$ for all $f \in \overline{\mathscr{L}}_{b}(\mathscr{Y})$. Since both $\overline{\mathrm{E}}^{\prime}$ and $\overline{\mathrm{E}}_{\text {ext }}$ satisfy E6 it then also follows that $\overline{\mathrm{E}}^{\prime}(f)=\lim _{c \rightarrow-\infty} \overline{\mathrm{E}}^{\prime}\left(f^{\vee c}\right)=\lim _{c \rightarrow-\infty} \overline{\mathrm{E}}_{\text {ext }}\left(f^{\vee c}\right)=\overline{\mathrm{E}}_{\text {ext }}(f)$ for all $f \in \overline{\mathscr{L}}(\mathscr{Y})$.

iii $\Rightarrow$ iii. Assume that $\overline{\mathrm{E}}$ is a coherent upper prevision such that $\overline{\mathrm{E}}^{\prime}=\overline{\mathrm{E}}_{\mathrm{ext}}$. Then, due to Proposition $7 \overline{\mathrm{E}}_{\mathrm{E} 14}$ is an upper expectation on $\overline{\mathscr{L}}_{b}(\mathscr{Y})$, which, by Proposition 2 implies that $\overline{\mathrm{E}}_{\mathrm{ext}}$-and therefore, also $\overline{\mathrm{E}}^{\prime}$-satisfies E6. To see that $\overline{\mathrm{E}}^{\prime}$ moreover satisfies E14] it suffices to recall that $\overline{\mathrm{E}}^{\prime}=\overline{\mathrm{E}}_{\mathrm{ext}}$ is an extension of $\overline{\mathrm{E}}_{\mathrm{E} 14}$ [because of Proposition[2] and that $\overline{\mathrm{E}}_{\mathrm{E} 14}$ satisfies E14][because of Proposition[6].

iii] $\Rightarrow$ i Assume that $\overline{\mathrm{E}}$ is a coherent upper prevision and $\overline{\mathrm{E}}^{\prime}$ satisfies E6 andE14, Then it suffices to prove that $\overline{\mathrm{E}}^{\prime}$ is an upper expectation on $\overline{\mathscr{L}}(\mathscr{Y})$, which can readily be inferred by combining Lemma 8 and Lemma 9

So if we assume our upper expectations to satisfyE6 we can justify their use on the entire domain $\overline{\mathscr{L}}(\mathscr{Y})$ by combining Walley's behavioural interpretation [19] with E6 and E14 For the local uncertainty models further on-which will simply be upper expectations on $\overline{\mathscr{L}}(\mathscr{X})$ with $\mathscr{X}$ a finite state space-, we would therefore typically be inclined to adopt this assumption, as we do in [19]. Moreover, it will turn out that imposing[E6 on the local models is necessary if we want to guarantee compatibility of local and global models; see Section 6 Nonetheless, we will generally not impose E6 on our local models here, because the current paper focusses on the mathematical properties of global game-theoretic upper expectations, which-apart from the compatibility with local models - are not affected by this additional continuity axiom. It can easily be seen that this is a consequence of the fact that the global game-theoretic upper expectation will only depend on the restrictions of the local models to $\overline{\mathscr{L}}_{b}(\mathscr{X})$.

\section{Game-theoretic Upper Expectations}

When mathematically modelling an uncertain process, one typically starts out with information about its local behaviour, that is, how its state $X_{k}$ will evolve from one time instant to the next. We will represent this information using so-called 'local' upper expectations; upper expectations on $\overline{\mathscr{L}}(\mathscr{X})$ where $\mathscr{X}$ is the finite state space of the process. Most often, one is interested in more general behaviour of the process, though, which 
confronts us with the question of how to combine the individual local assessments-represented by local upper expectations in our case-to obtain a single global uncertainty model. We consider one possible way of doing so; using the game-theoretic approach proposed by Shafer and Vovk. We will leave out most of the contextual discussion surrounding the following definitions. For more details, we refer the interested reader to [5, 12, 13, 19].

As explained in the introduction, we consider sequences $X_{1}, X_{2}, \ldots, X_{n}, \ldots$ of uncertain states that take values in a finite state space $\mathscr{X}$. We call any finite string $x_{1: n}:=\left(x_{1}, \ldots, x_{n}\right) \in \mathscr{X}_{1: n}:=\mathscr{X}^{n}$ of possible state values a situation and we denote the set of all situations by $\mathscr{X}^{*}:=\cup_{n \in \mathbb{N}_{0}} \mathscr{X}_{1: n}$. In particular, the unique empty string $x_{1: 0}$, denoted by $\square$, is called the initial situation, and $\mathscr{X}_{1: 0}:=\{\square\}$. In order to model the local behaviour of an uncertain process, we attach to each situation $x_{1: n} \in \mathscr{X}^{*}$ an upper expectation $\overline{\mathrm{Q}}_{x_{1: n}}$ on $\overline{\mathscr{L}}(\mathscr{X})$. Such an upper expectation $\overline{\mathrm{Q}}_{x_{1: n}}$-which we will call a local upper expectation-represents a subject's beliefs about what the next state of the process will be, given that it was in the states $x_{1} \cdots x_{n}$ at times $k=1$ through $k=n$. For instance, if we adopt a behavioural interpretation, the upper expectation $\overline{\mathrm{Q}}_{x_{1: n}}(f)$ for some $f \in \mathscr{L}(\mathscr{X})$ is the subject's infimum selling price for the gamble $f\left(X_{n+1}\right)$ that takes the value $f(x)$ if $X_{n+1}=x$ for any $x \in \mathscr{X}$, given that he observed the history $X_{1}=x_{1} \cdots X_{n}=x_{n}$. However, the local upper expectations could equally well be interpreted in terms of upper envelopes of linear expectations-which may in turn come from a set of probability mass functions. We do not enforce any interpretation for the local models $\overline{\mathrm{Q}}_{x_{1: n}}$; we simply assume that they are maps on $\overline{\mathscr{L}}(\mathscr{X})$ that satisfyE1 E5 A collection of local upper expectations $\overline{\mathrm{Q}}_{s}$, one for every $s \in \mathscr{X}^{*}$, is called an imprecise probabilities tree. Note that an imprecise Markov chain under epistemic irrelevance [4, 6, 8], for instance, corresponds to a special type of imprecise probabilities tree where, for any $n \in \mathbb{N}$ and any $x_{1: n} \in \mathscr{X}^{n}$, the local model $\overline{\mathrm{Q}}_{x_{1: n}}$ does not depend on the previous $n-1$ states $x_{1: n-1}$, nor on the time point $n$. So, in that case, we would have that $\overline{\mathrm{Q}}_{x_{1: n}}=\overline{\mathrm{Q}}_{x_{n}}$ for all $n \in \mathbb{N}$ and all $x_{1: n} \in \mathscr{X}^{n}$. We refer to [4, 9, 10] for more details on how the game-theoretic framework presented here can be implemented in an imprecise Markov chain setting.

In order to describe uncertain processes on a more global level, we will use the notion of a path $\omega$; an infinite sequence of state values. The set of all paths is called the sample space $\Omega:=\mathscr{X}^{\mathbb{N}}$. For any path $\omega \in \Omega$, the initial sequence that consists of its first $n$ state values is a situation in $\mathscr{X}_{1: n}$ that is denoted by $\omega^{n}$. The $n$-th state value is denoted by $\omega_{n} \in \mathscr{X}$. A collection of paths $A \subseteq \Omega$ is called an event. With any situation $x_{1: n}$, we associate the cylinder event $\Gamma\left(x_{1: n}\right):=\left\{\omega \in \Omega: \omega^{n}=x_{1: n}\right\}$ : the set of all paths $\omega \in \Omega$ that 'go through' the situation $x_{1: n}$. Sometimes, when it is clear from the context, we will also use the notation ' $x_{1: n}$ ' to denote the $\operatorname{set} \Gamma\left(x_{1: n}\right)$. For example, we will use $\mathbb{I}_{x_{1: n}}$ as a shorthand notation for $\mathbb{I}_{\Gamma\left(x_{1: n}\right)}$. Moreover, for any two extended real variables $g, h \in$ $\overline{\mathscr{L}}(\Omega)$ and any situation $s \in \mathscr{X}^{*}$, we use $g \leq_{s} f$ to denote that $g(\omega) \leq f(\omega)$ for all $\omega \in \Gamma(s)$, and similarly for $\geq_{s},>_{s}$ and $<_{s}$.

We will distinguish between local variables and global variables. Local variables are simply maps on the state space $\mathscr{X}$ and are typically interpreted as depending on the value of a single uncertain state $X_{n}$. They were already used before, for instance, when we introduced local upper expectations. In accordance with our earlier conventions, we use $\overline{\mathscr{L}}(\mathscr{X})$ to denote all local extended real variables, and similarly for $\overline{\mathscr{L}}_{b}(\mathscr{X})$ and $\mathscr{L}(\mathscr{X})$. Global variables, on the other hand, are maps on the sample space $\Omega$, and are therefore suitable for representing inferences that depend on the values of a large-possibly infinite-number of uncertain states. For example, the hitting time of a subset $A \subseteq \mathscr{X}$ is described by the global (extended real) variable $\tau_{A} \in \overline{\mathscr{L}}(\Omega)$ that takes the value 
$\tau_{A}(\omega):=\min \left\{n \in \mathbb{N}: \omega_{n} \in A\right\}$ for any $\omega \in \Omega$, and clearly depends on the values of an infinite number of subsequent states. We denote the set of all global extended real variables by $\overline{\mathbb{V}}:=\overline{\mathscr{L}}(\Omega)$, and similarly for $\overline{\mathbb{V}}_{b}:=\overline{\mathscr{L}}_{b}(\Omega)$ and $\mathbb{V}:=\mathscr{L}(\Omega)$. For any natural $k \leq \ell$, a special type of global variable-that is not necessarily extended realvalued-is the projection map $X_{k: \ell}$; for any path $\omega \in \Omega$, this variable assumes the value $X_{k: \ell}(\omega):=\left(\omega_{k}, \ldots, \omega_{\ell}\right)$. As such, for any $k \in \mathbb{N}, X_{k}=X_{k: k}$ can also be regarded as a type of global variable. For any $m, n \in \mathbb{N}$ and any map $f: \mathscr{X}^{n} \rightarrow \overline{\mathbb{R}}$, this allows us to write $f\left(X_{m: m+n-1}\right)$ to denote the extended real global variable defined by $f\left(X_{m: m+n-1}\right):=f \circ X_{m: m+n-1}$. In this way, we can elegantly associate a global variable $f\left(X_{n}\right)$ with any local variable $f: \mathscr{X} \rightarrow \overline{\mathbb{R}}$ and any discrete time point $n \in \mathbb{N}$.

Our aim now is to combine the local upper expectations $\overline{\mathrm{Q}}_{s}$ - which only tell us something about state transitionsand construct a global uncertainty model in the form of a single upper expectation on the global variables $\overline{\mathbb{V}}$ (and conditional on the situations $\mathscr{X}^{*}$ ). A crucial tool to do so, is the notion of a supermartingale; a special type of process.

Any map $\mathscr{P}$ on $\mathscr{X}^{*}$ is called a process. An extended real(-valued) process $\mathscr{P}$ is called bounded below if there is some $M \in \mathbb{R}$ such that $\mathscr{P}(s) \geq M$ for all $s \in \mathscr{X}^{*}$. Furthermore, with any situation $s \in \mathscr{X}^{*}$ and any extended real process $\mathscr{P}$, we can associate the local variable $\mathscr{P}(s \cdot) \in \overline{\mathscr{L}}(\mathscr{X})$ defined by $\mathscr{P}(s \cdot)(x):=\mathscr{P}(s x)$ for all $x \in \mathscr{X}$. The extended real variables $\lim \inf \mathscr{P} \in \overline{\mathbb{V}}$ and $\lim \sup \mathscr{P} \in \overline{\mathbb{V}}$, will be defined by

$$
\liminf \mathscr{P}(\omega):=\liminf _{n \rightarrow+\infty} \mathscr{P}\left(\omega^{n}\right) \text { and } \limsup \mathscr{P}(\omega):=\limsup _{n \rightarrow+\infty} \mathscr{P}\left(\omega^{n}\right)
$$

for all $\omega \in \Omega$. If $\lim \inf \mathscr{P}=\lim \sup \mathscr{P}$, we denote their common value by $\lim \mathscr{P}$.

For a given imprecise probabilities tree, a supermartingale $\mathscr{M}$ is an extended real process such that $\overline{\mathrm{Q}}_{s}(\mathscr{M}(s \cdot)) \leq$ $\mathscr{M}(s)$ for all $s \in \mathscr{X}^{*}$. So a supermartingale is an extended real process that, according to the local models $\overline{\mathrm{Q}}_{s}$, is expected to decrease. When adopting a behavioural interpretation, supermartingales can be seen to represent betting strategies that are allowed by our subject. Roughly speaking, the condition that $\overline{\mathrm{Q}}_{s}(\mathscr{M}(s \cdot)) \leq \mathscr{M}(s)$ with $s=x_{1: n}$ then means that our subject— for the sake of simplicity, we ignore the subtlety about the extended realvaluedness - is willing to receive the price $\mathscr{M}\left(x_{1: n}\right)$ for giving away the uncertain variable $\mathscr{M}\left(x_{1: n} X_{n+1}\right)$ that will be evaluated in the next time instant. Hence, if we take him up on his commitments, we can pay him $\mathscr{M}\left(x_{1: n}\right)$ to receive $\mathscr{M}\left(x_{1: n} X_{n+1}\right)$. The next time instant, if the state of the process turns out to be $x_{n+1} \in \mathscr{X}$, we obtain the-possibly negative-payoff $\mathscr{M}\left(x_{1: n+1}\right)$. By repeating this procedure, we find that the supermartingale $\mathscr{M}$ represents a possible evolution of our capital when we would gamble against the subject. We will denote the set of all bounded below supermartingales for a given imprecise probabilities tree by $\overline{\mathbb{M}}_{\mathrm{b}}$.

In the framework of Shafer and Vovk, the role of our subject above is taken up by a player called 'Forecaster', whereas supermartingales represent possible betting strategies for a second player called 'Skeptic'. Given this game-theoretic setting, they consider the following question: How can Skeptic use Forecaster's assessments to determine selling and buying prices for a gamble $f$ whose uncertain payoff depends on the process state at multiple or even an infinite number of time instances? Shafer and Vovk argue that Skeptic should certainly agree on selling $f$ for a price $\alpha$ such that, if Skeptic starts with an initial capital $\alpha$ and gambles in an appropriate way against Forecaster, he will end up with a higher capital than the payoff $f(\omega)$ corresponding to $f$ irrespectively of the path $\omega \in \Omega$ taken by the process. Indeed, selling $f$ for a price $\alpha$ means that Skeptic receives $\alpha-f$. If Skeptic is then able to turn the initial capital $\alpha$ into a final capital $K$ such that $K(\omega) \geq f(\omega)$ for all paths $\omega \in \Omega$, his net payoff 
$K-f$ is non-negative for all $\omega$. Hence, Skeptic should accept the transaction of selling $f$ for $\alpha$. The infimum of these prices $\alpha$ is what Shafer and Vovk then call the (global) game-theoretic upper expectation of $f$.

More formally, given an imprecise probabilities tree consisting of local upper expectations $\overline{\mathrm{Q}}_{s}$ for all $s \in \mathscr{X}^{*}$, we use its compatible set of bounded below supermartingales $\overline{\mathbb{M}}_{\mathrm{b}}$ to define the corresponding (global) gametheoretic upper expectation $\overline{\mathrm{E}}_{\mathrm{V}}$ as follows.

Definition 3. For any imprecise probabilities tree, the corresponding (global) game-theoretic upper expectation $\overline{\mathrm{E}}_{\mathrm{V}}(\cdot \cdot \cdot): \overline{\mathbb{V}} \times \mathscr{X}^{*} \rightarrow \overline{\mathbb{R}}$ is defined by

$$
\overline{\mathrm{E}}_{\mathrm{V}}(f \mid s):=\inf \left\{\mathscr{M}(s): \mathscr{M} \in \overline{\mathbb{M}}_{\mathrm{b}} \text { and } \liminf \mathscr{M} \geq_{s} f\right\} \text { for all } f \in \overline{\mathbb{V}} \text { and all } s \in \mathscr{X}^{*} .
$$

The game-theoretic lower expectation $\underline{\mathrm{E}}_{\mathrm{V}}(\cdot \cdot \cdot): \overline{\mathbb{V}} \times \mathscr{X}^{*} \rightarrow \overline{\mathbb{R}}$ is defined by the conjugacy relation $\underline{\mathrm{E}}_{\mathrm{V}}(f \mid s):=$ $-\overline{\mathrm{E}}_{\mathrm{V}}(-f \mid s)$ for all $f \in \overline{\mathbb{V}}$ and all $s \in \mathscr{X}^{*}$. We will show later in Corollary 24 that, for any $s \in \mathscr{X}^{*}$, the map $\overline{\mathrm{E}}_{\mathrm{V}}(\cdot \mid s): \overline{\mathbb{V}} \rightarrow \overline{\mathbb{R}}$ satisfies E1 E5 which justifies calling $\overline{\mathrm{E}}_{\mathrm{V}}$ an upper expectation. Mimicking the link between traditional expectations and probabilities, we call $\overline{\mathrm{P}}_{\mathrm{V}}(A \mid s):=\overline{\mathrm{E}}_{\mathrm{V}}\left(\mathbb{I}_{A} \mid s\right)$, for any $A \subseteq \Omega$ and any $s \in \mathscr{X}^{*}$, the game-theoretic upper probability of the event $A$ conditional on the situation $s$. Similarly, we call $\underline{\mathrm{P}}_{\mathrm{V}}(A \mid s):=\underline{\mathrm{E}}_{\mathrm{V}}\left(\mathbb{I}_{A} \mid s\right)$ the gametheoretic lower probability of $A$ conditional on $s$. We will also let $\overline{\mathrm{E}}_{\mathrm{V}}(f):=\overline{\mathrm{E}}_{\mathrm{V}}(f \mid \square)$ and $\underline{\mathrm{E}}_{\mathrm{V}}(f):=\underline{\mathrm{E}}_{\mathrm{V}}(f \mid \square)$ for all $f \in \overline{\mathbb{V}}$.

Note that $\overline{\mathrm{E}}_{\mathrm{V}}$ does not depend on the values of the local models $\overline{\mathrm{Q}}_{s}$ on $\overline{\mathscr{L}}(\mathscr{X}) \backslash \overline{\mathscr{L}}_{b}(\mathscr{X})$, because the infimum in Definition 3 is taken over supermartingales that are bounded below. This confirms our earlier claim in Section2 where we said that, as far as the global upper expectation $\overline{\mathrm{E}}_{\mathrm{V}}$ is concerned, we can assume without loss of generality that the local models $\overline{\mathrm{Q}}_{s}$ additionally satisfy Shafer and Vovk's axioms E2' E4' Our reason for adopting this particular definition, where only bounded below supermartingales are considered, will be discussed in Section 8. Intuitively, however, one could interpret this assumption as a concretisation of the fact that a subject (e.g. Skeptic) cannot borrow an infinite or even unbounded amount of money.

\section{Basic Properties of Game-Theoretic Upper Expectations}

We start by establishing some basic, yet essential properties of game-theoretic upper expectations. The main ones are an extended version of coherence, partial compatibility with the local upper expectations and a law of iterated upper expectations. Most of these results are not entirely new and have already been proved in a slightly different setting; our contribution then consists in adapting their proofs to our setting. We start with the following two, rather abstract lemmas about supermartingales.

Lemma 11. Consider any $\mathscr{M} \in \overline{\mathbb{M}}_{\mathrm{b}}$ and any situation $s \in \mathscr{X}^{*}$. Then

$$
\mathscr{M}(s) \geq \inf _{\omega \in \Gamma(s)} \lim \sup \mathscr{M}(\omega) \geq \inf _{\omega \in \Gamma(s)} \liminf \mathscr{M}(\omega) .
$$

Proof. The proof is similar to that of 44 , Lemma 1], where instead real supermartingales were used. Since $\mathscr{M}$ is a bounded below supermartingale, we have that $\overline{\mathrm{Q}}_{s}(\mathscr{M}(s \cdot)) \leq \mathscr{M}(s)$, which byE7]implies that $\inf _{x \in \mathscr{X}} \mathscr{M}(s x) \leq$ $\mathscr{M}(s)$. Hence, since $\mathscr{X}$ is finite, there is at least one $x \in \mathscr{X}$ such that $\mathscr{M}(s x) \leq \mathscr{M}(s)$. Repeating this argument over and over again, leads us to the conclusion that there is some $\omega \in \Gamma(s)$ such that lim $\sup _{n \rightarrow+\infty} \mathscr{M}\left(\omega^{n}\right) \leq \mathscr{M}(s)$ and therefore also $\inf _{\omega \in \Gamma(s)} \lim \sup \mathscr{M}(\omega) \leq \mathscr{M}(s)$. The rest of the proof is now trivial. 
Lemma 12. Consider any countable collection $\left\{\mathscr{M}_{n}\right\}_{n \in \mathbb{N}_{0}}$ of supermartingales that have a common lower bound, and any countable collection of non-negative real numbers $\left\{\lambda_{n}\right\}_{n \in \mathbb{N}_{0}}$ such that $\sum_{n \in \mathbb{N}_{0}} \lambda_{n}$ is a real number $\lambda$. Then $\mathscr{M}:=\sum_{n \in \mathbb{N}_{0}} \lambda_{n} \mathscr{M}_{n}$ is again a bounded below supermartingale. If, moreover, all $\mathscr{M}_{n}$ are non-negative, then so is $\mathscr{M}$.

Proof. We only prove the first statement, as the second is then trivially true. Since all $\mathscr{M}_{n}$ have a common lower bound, say $B \in \mathbb{R}$, the processes $\mathscr{M}_{n}-B$ will be non-negative and therefore, because all reals $\lambda_{n}$ are also nonnegative, the sum $\sum_{n \in \mathbb{N}_{0}} \lambda_{n}\left[\mathscr{M}_{n}(s)-B\right]$ exists and is non-negative for all $s \in \mathscr{X}^{*}$. Then, in order to see that $\mathscr{M}$ is well-defined, note that

$$
\begin{aligned}
\sum_{n \in \mathbb{N}_{0}} \lambda_{n}\left[\mathscr{M}_{n}(s)-B\right]+\lambda B & =\lim _{n \rightarrow+\infty}\left(\sum_{i=0}^{n} \lambda_{i}\left[\mathscr{M}_{i}(s)-B\right]+\sum_{i=0}^{n} \lambda_{i} B\right) \\
& =\lim _{n \rightarrow+\infty} \sum_{i=0}^{n}\left(\lambda_{i}\left[\mathscr{M}_{i}(s)-B\right]+\lambda_{i} B\right)=\sum_{n \in \mathbb{N}_{0}} \lambda_{n} \mathscr{M}_{n}(s)=: \mathscr{M}(s),
\end{aligned}
$$

for all $s \in \mathscr{X}^{*}$, where the first step takes into account that $\lambda B$ is real and the third step takes into account that all $\lambda_{i}$ and $B$ are real. The equality above, together with the non-negativity of $\sum_{n \in \mathbb{N}_{0}} \lambda_{n}\left[\mathscr{M}_{n}(s)-B\right]$ immediately shows that $\mathscr{M}$ is bounded below by the real $\lambda B$. It also shows that $\mathscr{M}$ is a supermartingale. Indeed, for any $s \in \mathscr{X}^{*}$, we find that

$$
\begin{aligned}
& \overline{\mathrm{Q}}_{s}(\mathscr{M}(s \cdot)) \stackrel{\underline{\underline{5}}}{\underline{\mathrm{Q}}}{ }_{s}\left(\sum_{n \in \mathbb{N}_{0}} \lambda_{n}\left[\mathscr{M}_{n}(s \cdot)-B\right]+\lambda B\right) \stackrel{\text { 圆}}{=} \overline{\mathrm{Q}}_{s}\left(\sum_{n \in \mathbb{N}_{0}} \lambda_{n}\left[\mathscr{M}_{n}(s \cdot)-B\right]\right)+\lambda B \\
& \leq \sum_{n \in \mathbb{N}_{0}} \overline{\mathrm{Q}}_{s}\left(\lambda_{n}\left[\mathscr{M}_{n}(s \cdot)-B\right]\right)+\lambda B \stackrel{\mathrm{EQ}}{=} \sum_{n \in \mathbb{N}_{0}} \lambda_{n} \overline{\mathrm{Q}}_{s}\left(\mathscr{M}_{n}(s \cdot)-B\right)+\lambda B \\
& \stackrel{\text { 贯8 }}{=} \sum_{n \in \mathbb{N}_{0}} \lambda_{n}\left(\overline{\mathrm{Q}}_{s}\left(\mathscr{M}_{n}(s \cdot)\right)-B\right)+\lambda B=\sum_{n \in \mathbb{N}_{0}} \lambda_{n} \overline{\mathrm{Q}}_{s}\left(\mathscr{M}_{n}(s \cdot)\right) \leq \sum_{n \in \mathbb{N}_{0}} \lambda_{n} \mathscr{M}_{n}(s)=\mathscr{M}(s),
\end{aligned}
$$

where we were allowed to apply E8 and E9 because $\sum_{n \in \mathbb{N}_{0}} \lambda_{n}\left[\mathscr{M}_{n}(s \cdot)-B\right]$, all $\mathscr{M}_{n}(s \cdot)-B$ and all $\mathscr{M}_{n}(s \cdot)$ are bounded below, where the first inequality followed from Proposition 4 and where the last inequality followed from the non-negativity of all $\lambda_{n}$ and the fact that all $\mathscr{M}_{n}$ are supermartingales.

The following result states that $\overline{\mathrm{E}}_{\mathrm{V}}$ satisfies a version of the coherence axioms for global extended real variables. A first version of the result was stated in [12, Chapter 8], yet, our proof is very similar to that of [4, Prop. 14]: we adapt it here to the fact that our bounded below supermartingales take values in $\overline{\mathbb{R}}$ rather than $\mathbb{R}$.

Proposition 13. For all extended real variables $f, g \in \overline{\mathbb{V}}$, all $\lambda \in \mathbb{R}_{\geq 0}$, all $\mu \in \mathbb{R}$ and all situations $s \in \mathscr{X}^{*}, \overline{\mathrm{E}}_{\mathrm{V}}$ satisfies

V1. $\overline{\mathrm{E}}_{\mathrm{V}}(f \mid s) \leq \sup _{\omega \in \Gamma(s)} f(\omega)$;

V2. $\overline{\mathrm{E}}_{\mathrm{V}}(f+g \mid s) \leq \overline{\mathrm{E}}_{\mathrm{V}}(f \mid s)+\overline{\mathrm{E}}_{\mathrm{V}}(g \mid s)$;

V3. $\overline{\mathrm{E}}_{\mathrm{V}}(\lambda f \mid s)=\lambda \overline{\mathrm{E}}_{\mathrm{V}}(f \mid s)$.

V4. $f \leq_{s} g \Rightarrow \overline{\mathrm{E}}_{\mathrm{V}}(f \mid s) \leq \overline{\mathrm{E}}_{\mathrm{V}}(g \mid s)$; 
V5. $\inf _{\omega \in \Gamma(s)} f(\omega) \leq \underline{\mathrm{E}}_{\mathrm{V}}(f \mid s) \leq \overline{\mathrm{E}}_{\mathrm{V}}(f \mid s) \leq \sup _{\omega \in \Gamma(s)} f(\omega)$

V6. $\overline{\mathrm{E}}_{\mathrm{V}}(f+\mu \mid s)=\overline{\mathrm{E}}_{\mathrm{V}}(f \mid s)+\mu$.

Proof. V1 If $\sup _{\omega \in \Gamma(s)} f(\omega)=+\infty$, the inequality is trivially satisfied. If this is not the case, consider any real $M \geq \sup _{\omega \in \Gamma(s)} f(\omega)$ and the real process $\mathscr{M}$ that assumes the constant value $M$. Then clearly $\mathscr{M}$ is a bounded below supermartingale and moreover $\liminf \mathscr{M}(\omega)=M \geq f(\omega)$ for all $\omega \in \Gamma(s)$. Hence, Definition 3 implies that $\overline{\mathrm{E}}_{\mathrm{V}}(f \mid s) \leq \mathscr{M}(s)=M$. Since this is true for every real $M \geq \sup _{\omega \in \Gamma(s)} f(\omega)$, $\mathrm{V1}$ follows.

V2 If either $\overline{\mathrm{E}}_{\mathrm{V}}(f \mid s)$ or $\overline{\mathrm{E}}_{\mathrm{V}}(g \mid s)$ equals $+\infty$, then the inequality is trivially true. So suppose that $\overline{\mathrm{E}}_{\mathrm{V}}(f \mid s)<+\infty$ and $\overline{\mathrm{E}}_{\mathrm{V}}(g \mid s)<+\infty$ and consider any real $c_{1}>\overline{\mathrm{E}}_{\mathrm{V}}(f \mid s)$ and any real $c_{2}>\overline{\mathrm{E}}_{\mathrm{V}}(g \mid s)$. Then there are two bounded below supermartingales $\mathscr{M}_{1}$ and $\mathscr{M}_{2}$ such that $\mathscr{M}_{1}(s) \leq c_{1}$ and $\mathscr{M}_{2}(s) \leq c_{2}$ and moreover liminf $\mathscr{M}_{1} \geq_{s} f$ and $\liminf \mathscr{M}_{2} \geq_{s} g$. Now consider the extended real process $\mathscr{M}:=\mathscr{M}_{1}+\mathscr{M}_{2}$. Then $\mathscr{M}$ is a bounded below supermartingale because of Lemma 12 which we can apply because $\mathscr{M}_{1}$ and $\mathscr{M}_{2}$ are both bounded below and hence have a common lower bound [note that the countable sum in Lemma 12 can be turned into a finite sum by setting all remaining supermartingales equal to zero]. Moreover, we will show that $\lim \inf \left(\mathscr{M}_{1}+\mathscr{M}_{2}\right) \geq$ $\liminf \mathscr{M}_{1}+\liminf \mathscr{M}_{2}$ and therefore that liminf $\mathscr{M} \geq_{s} f+g$, which, by Definition 3 implies that $\overline{\mathrm{E}}_{\mathrm{V}}(f+g \mid s) \leq$ $\mathscr{M}(s)=\mathscr{M}_{1}(s)+\mathscr{M}_{2}(s) \leq c_{1}+c_{2}$. Since this then holds for any real $c_{1}>\overline{\mathrm{E}}_{\mathrm{V}}(f \mid s)$ and any real $c_{2}>\overline{\mathrm{E}}_{\mathrm{V}}(g \mid s)$, it follows that $\overline{\mathrm{E}}_{\mathrm{V}}(f+g \mid s) \leq \overline{\mathrm{E}}_{\mathrm{V}}(f \mid s)+\overline{\mathrm{E}}_{\mathrm{V}}(g \mid s)$.

So consider any $\omega \in \Omega$ and any real $\alpha_{1}$ and $\alpha_{2}$ such that $\liminf \mathscr{M}_{1}(\omega)>\alpha_{1}$ and $\liminf \mathscr{M}_{2}(\omega)>\alpha_{2}$. This is always possible because $\mathscr{M}_{1}$ and $\mathscr{M}_{2}$ are bounded below. Then there are two natural numbers $N_{1}$ and $N_{2}$ such that $\mathscr{M}_{1}\left(\omega^{n_{1}}\right) \geq \alpha_{1}$ and $\mathscr{M}_{2}\left(\omega^{n_{2}}\right) \geq \alpha_{2}$ for all $n_{1} \geq N_{1}$ and all $n_{2} \geq N_{2}$. Hence, we have that $\mathscr{M}_{1}\left(\omega^{n}\right)+\mathscr{M}_{2}\left(\omega^{n}\right) \geq$ $\alpha_{1}+\alpha_{2}$ for all $n \geq \max \left\{N_{1}, N_{2}\right\}$, implying that $\liminf \left(\mathscr{M}_{1}+\mathscr{M}_{2}\right)(\omega) \geq \alpha_{1}+\alpha_{2}$. Since this holds for any real $\alpha_{1}$ and $\alpha_{2}$ such that $\liminf \mathscr{M}_{1}(\omega)>\alpha_{1}$ and $\liminf \mathscr{M}_{2}(\omega)>\alpha_{2}$, we indeed find that $\liminf \left(\mathscr{M}_{1}+\mathscr{M}_{2}\right)(\omega) \geq \liminf \mathscr{M}_{1}(\omega)+$ $\liminf \mathscr{M}_{2}(\omega)$.

V3. For $\lambda \in \mathbb{R}_{>0}$, it suffices to note that $\mathscr{M}$ is a bounded below supermartingale such that liminf $\mathscr{M} \geq_{s} f$ if and only if $\lambda \mathscr{M}$ is a bounded below supermartingale such that liminf $\lambda \mathscr{M} \geq_{s} \lambda f$. If $\lambda=0$, then $\lambda \overline{\mathrm{E}}_{\mathrm{V}}(f \mid s)=0$ because $(+\infty) \cdot 0=(-\infty) \cdot 0=0$. To see that also $\overline{\mathrm{E}}_{\mathrm{V}}(\lambda f \mid s)=0$, start by noting that $\lambda f=0$ and hence, because of V1] $\overline{\mathrm{E}}_{\mathrm{V}}(\lambda f \mid s) \leq 0$. That $\overline{\mathrm{E}}_{\mathrm{V}}(\lambda f \mid s)<0$ is impossible, follows from Lemma11 and Definition 3 Hence, we indeed have that $\overline{\mathrm{E}}_{\mathrm{V}}(\lambda f \mid s)=0$.

V4. Consider any two $f, g \in \overline{\mathbb{V}}$ such that $f \leq_{s} g$. Then for any $\mathscr{M} \in \overline{\mathbb{M}}_{\mathrm{b}}$ such that liminf $\mathscr{M} \geq_{s} g$, we also have that $\liminf \mathscr{M} \geq_{s} f$, and hence, by Definition $3, \overline{\mathrm{E}}_{\mathrm{V}}(f \mid s) \leq \overline{\mathrm{E}}_{\mathrm{V}}(g \mid s)$.

V5. The first and third inequality follow trivially from V1 and the definition of the conjugate lower expectation $\underline{\mathrm{E}}_{\mathrm{V}}$. To prove the second inequality, assume ex absurdo that $\underline{\mathrm{E}}_{\mathrm{V}}(f \mid s)>\overline{\mathrm{E}}_{\mathrm{V}}(f \mid s)$. Then $0>\overline{\mathrm{E}}_{\mathrm{V}}(f \mid s)-\underline{\mathrm{E}}_{\mathrm{V}}(f \mid s)$ which, by $\mathrm{V} 2$ and the definition of the conjugate lower expectation $\underline{\mathrm{E}}_{\mathrm{V}}$, implies that $0>\overline{\mathrm{E}}_{\mathrm{V}}(f+(-f) \mid s)$. Since, according to our convention, the extended real variable $f+(-f)$ only assumes values in $\{0,+\infty\}$, we have that $f+(-f) \geq 0$ and therefore, by $\mathrm{V4}$ and $\mathrm{V3}$, that $\overline{\mathrm{E}}_{\mathrm{V}}(f+(-f) \mid s) \geq \overline{\mathrm{E}}_{\mathrm{V}}(0 \mid s)=0$. This is a contradiction.

V6. For any $\mathscr{M} \in \overline{\mathbb{M}}_{\mathrm{b}}$ such that liminf $\mathscr{M} \geq_{s} f+\mu$, we have that $\mathscr{M}-\mu \in \overline{\mathbb{M}}_{\mathrm{b}}$ because of E8 and moreover $\liminf (\mathscr{M}-\mu) \geq_{s} f$. Hence, $\overline{\mathrm{E}}_{\mathrm{V}}(f \mid s) \leq \mathscr{M}(s)-\mu$ and therefore also $\overline{\mathrm{E}}_{\mathrm{V}}(f \mid s)+\mu \leq \mathscr{M}(s)-\mu+\mu=\mathscr{M}(s)$. Since this holds for any $\mathscr{M} \in \overline{\mathbb{M}}_{\mathrm{b}}$ such that liminf $\mathscr{M} \geq_{s} f+\mu$, we have that $\overline{\mathrm{E}}_{\mathrm{V}}(f \mid s)+\mu \leq \overline{\mathrm{E}}_{\mathrm{V}}(f+\mu \mid s)$. By applying this inequality to $f^{\prime}=f+\mu$ and $\mu^{\prime}=-\mu$, we also find that $\overline{\mathrm{E}}_{\mathrm{V}}(f+\mu \mid s)-\mu \leq \overline{\mathrm{E}}_{\mathrm{V}}(f \mid s)$. 
In order to formulate our next result, we require the concept of an $n$-measurable variable. For a given $n \in \mathbb{N}_{0}$, we call a global variable $f n$-measurable if it is constant on the cylinder events $\Gamma\left(x_{1: n}\right)$ for all $x_{1: n} \in \mathscr{X}_{1: n}$, that is, if $f=\tilde{f}\left(X_{1: n}\right)$ for some map $\tilde{f}$ on $\mathscr{X}^{n}$. We will then also use the notation $f\left(x_{1: n}\right)$ for its constant value $f(\omega)$ on all paths $\omega \in \Gamma\left(x_{1: n}\right)$. Similarly, for a global variable $f$ that only depends on the $n$-th state $X_{n}$, we will use $f\left(x_{n}\right)$ to denote its constant value on the event $\left\{\omega \in \Omega: \omega_{n}=x_{n}\right\}$. We call a global variable $f \in \overline{\mathbb{V}}$ finitary if it is $n$-measurable for some $n \in \mathbb{N}_{0}$. With any situation $x_{1: n} \in \mathscr{X}^{*}$ and any $(n+1)$-measurable extended real variable $f$, we now associate a local variable $f\left(x_{1: n} \cdot\right)$ defined by $f\left(x_{1: n}\right)\left(x_{n+1}\right):=f\left(x_{1: n+1}\right)$ for all $x_{n+1} \in \mathscr{X}$. On the other hand, for any extended real process $\mathscr{P}$, we will define the global variable $\mathscr{P}\left(X_{1: n}\right):=\mathscr{P} \circ X_{1: n}$ that only depends of the first $n$ states, and is therefore finitary.

Our proof of Proposition 14 also requires the following additional notation and terminology. For any two situations $s, t \in \mathscr{X}^{*}$, we write that $s \sqsubseteq t$, or equivalently that $t \sqsupseteq s$, when every path that goes through $t$ also goes through $s$. In that case we say that $s$ precedes $t$ or that $t$ follows $s$. When $s \sqsubseteq t$ and $s \neq t$, we write $s \sqsubset t$ and similarly for the relation $\sqsupset$. When neither $s \sqsubseteq t$ nor $t \sqsubseteq s$, we say that $s$ and $t$ are incomparable.

Proposition 14 (Partial compatibility with local models). Consider any situation $x_{1: n} \in \mathscr{X}^{*}$ and any $(n+1)$ measurable extended real variable $f$ that is bounded below. Then,

$$
\overline{\mathrm{E}}_{\mathrm{V}}\left(f \mid x_{1: n}\right)=\overline{\mathrm{Q}}_{x_{1: n}}\left(f\left(x_{1: n} \cdot\right)\right) .
$$

Proof. Our proof is similar to that of [4, Corollary 3]. Consider any $\mathscr{M} \in \overline{\mathbb{M}}_{\mathrm{b}}$ such that liminf $\mathscr{M} \geq_{x_{1: n}} f$. Then it follows from Lemma 11 that, for all $x_{n+1} \in \mathscr{X}$,

$$
\mathscr{M}\left(x_{1: n+1}\right) \geq \inf _{\omega \in \Gamma\left(x_{1: n+1}\right)} \liminf \mathscr{M}(\omega) \geq \inf _{\omega \in \Gamma\left(x_{1: n+1}\right)} f(\omega)=f\left(x_{1: n+1}\right) .
$$

Hence, we have that $\mathscr{M}\left(x_{1: n} \cdot\right) \geq f\left(x_{1: n} \cdot\right)$, which implies by E4 and the supermartingale character of $\mathscr{M}$ that

$$
\mathscr{M}\left(x_{1: n}\right) \geq \overline{\mathrm{Q}}_{x_{1: n}}\left(\mathscr{M}\left(x_{1: n} \cdot\right)\right) \geq \overline{\mathrm{Q}}_{x_{1: n}}\left(f\left(x_{1: n} \cdot\right)\right) .
$$

Since this holds for any $\mathscr{M} \in \overline{\mathbb{M}}_{\mathrm{b}}$ such that $\liminf \mathscr{M} \geq_{x_{1: n}} f$, it follows from Definition 3 that $\overline{\mathrm{E}}_{\mathrm{V}}\left(f \mid x_{1: n}\right) \geq$ $\overline{\mathrm{Q}}_{x_{1: n}}\left(f\left(x_{1: n}\right)\right)$. To see that the inequality is an equality, consider the extended real process $\mathscr{M}$ defined by $\mathscr{M}(s):=$ $\overline{\mathrm{Q}}_{x_{1: n}}\left(f\left(x_{1: n}\right)\right)$ for all $s \not \supset x_{1: n}$, and by $\mathscr{M}(s):=f\left(x_{1: n+1}\right)$ for any $s \in \mathscr{X}^{*}$ such that $s \sqsupseteq x_{1: n+1}$ for some $x_{n+1} \in \mathscr{X}$. Then $\mathscr{M}$ is bounded below because $f$ is bounded below and $\overline{\mathrm{Q}}_{x_{1: n}}$ satisfies E7 It is also a supermartingale because $\overline{\mathrm{Q}}_{x_{1: n}}\left(\mathscr{M}\left(x_{1: n} \cdot\right)\right)=\overline{\mathrm{Q}}_{x_{1: n}}\left(f\left(x_{1: n} \cdot\right)\right)=\mathscr{M}\left(x_{1: n}\right)$ and, for all $s \neq x_{1: n}, \overline{\mathrm{Q}}_{s}(\mathscr{M}(s \cdot))=\mathscr{M}(s)$ because of E7 and the fact that $\mathscr{M}(s \cdot)$ is constant and equal to $\mathscr{M}(s)$. It is moreover easy to see that $\liminf \mathscr{M} \geq_{x_{1: n}} f$ is guaranteed because $f$ is $(n+1)$-measurable.

We will show later on that this compatibility can be extended to the entire domain of the local models $\overline{\mathrm{Q}}_{s}$ provided that they additionally satisfyE6

The following important result is an imprecise generalisation of the well-known 'law of iterated expectations'. The idea of the proof goes back to [12, Proposition 8.7], yet, our proof is more similar to that of [4, Theorem 16].

Theorem 15 (Law of iterated upper expectations). For any $f \in \overline{\mathbb{V}}$ and any $x_{1: n} \in \mathscr{X}^{*}$, we have that

$$
\overline{\mathrm{E}}_{\mathrm{V}}\left(f \mid x_{1: n}\right)=\overline{\mathrm{E}}_{\mathrm{V}}\left(\overline{\mathrm{E}}_{\mathrm{V}}\left(f \mid x_{1: n} X_{n+1}\right) \mid x_{1: n}\right) .
$$


Proof. Fix any $f \in \overline{\mathbb{V}}$ and any $x_{1: n} \in \mathscr{X}^{*}$. We first show that $\overline{\mathrm{E}}_{\mathrm{V}}\left(\overline{\mathrm{E}}_{\mathrm{V}}\left(f \mid x_{1: n} X_{n+1}\right) \mid x_{1: n}\right) \leq \overline{\mathrm{E}}_{\mathrm{V}}\left(f \mid x_{1: n}\right)$. If $\overline{\mathrm{E}}_{\mathrm{V}}\left(f \mid x_{1: n}\right)=$ $+\infty$, this is trivially satisfied. If not, then for any fixed real $\alpha>\overline{\mathrm{E}}_{\mathrm{V}}\left(f \mid x_{1: n}\right)$ there is a bounded below supermartingale $\mathscr{M}$ such that $\mathscr{M}\left(x_{1: n}\right) \leq \alpha$ and liminf $\mathscr{M} \geq_{x_{1: n}} f$. Then it is clear that, for all $x_{n+1} \in \mathscr{X}$, $\liminf \mathscr{M} \geq_{x_{1: n+1}} f$, and hence $\overline{\mathrm{E}}_{\mathrm{V}}\left(f \mid x_{1: n+1}\right) \leq \mathscr{M}\left(x_{1: n+1}\right)$ by Definition3, Let $\mathscr{M}^{\prime}$ be the process that is equal to $\mathscr{M}$ for all situations that precede $x_{1: n}$ or are incomparable with $x_{1: n}$, and that is equal to the constant $\mathscr{M}\left(x_{1: n+1}\right)$ for all situations that follow $x_{1: n+1}$ for some $x_{n+1} \in \mathscr{X}$. Clearly, $\mathscr{M}^{\prime}$ is again a bounded below supermartingale and, because of the reasoning above, $\overline{\mathrm{E}}_{\mathrm{V}}\left(f \mid x_{1: n} X_{n+1}\right) \leq \mathscr{M}\left(x_{1: n} X_{n+1}\right)={ }_{x_{1: n}} \liminf \mathscr{M}^{\prime}$. Hence, it follows from Definition 3 that $\overline{\mathrm{E}}_{\mathrm{V}}\left(\overline{\mathrm{E}}_{\mathrm{V}}\left(f \mid x_{1: n} X_{n+1}\right) \mid x_{1: n}\right) \leq \mathscr{M}^{\prime}\left(x_{1: n}\right)=\mathscr{M}\left(x_{1: n}\right) \leq \alpha$. Since this holds for any real $\alpha>\overline{\mathrm{E}}_{\mathrm{V}}\left(f \mid x_{1: n}\right)$, we indeed have that $\overline{\mathrm{E}}_{\mathrm{V}}\left(\overline{\mathrm{E}}_{\mathrm{V}}\left(f \mid x_{1: n} X_{n+1}\right) \mid x_{1: n}\right) \leq \overline{\mathrm{E}}_{\mathrm{V}}\left(f \mid x_{1: n}\right)$.

We now prove the other inequality. Again, if $\overline{\mathrm{E}}_{\mathrm{V}}\left(\overline{\mathrm{E}}_{\mathrm{V}}\left(f \mid x_{1: n} X_{n+1}\right) \mid x_{1: n}\right)=+\infty$ it trivially holds, so we can assume it to be real or equal to $-\infty$. Fix any real $\alpha>\overline{\mathrm{E}}_{\mathrm{V}}\left(\overline{\mathrm{E}}_{\mathrm{V}}\left(f \mid x_{1: n} X_{n+1}\right) \mid x_{1: n}\right)$ and any $\epsilon \in \mathbb{R}_{>0}$. Then there must be a bounded below supermartingale $\mathscr{M}$ such that $\mathscr{M}\left(x_{1: n}\right) \leq \alpha$ and $\liminf \mathscr{M} \geq_{x_{1: n}} \overline{\mathrm{E}}_{\mathrm{V}}\left(f \mid x_{1: n} X_{n+1}\right)$. Consider any such bounded below supermartingale. Then for any $x_{n+1} \in \mathscr{X}$, we have that liminf $\mathscr{M} \geq_{x_{1: n+1}} \overline{\mathrm{E}}_{\mathrm{V}}\left(f \mid x_{1: n+1}\right)$, which by Lemma 11 implies that $\mathscr{M}\left(x_{1: n+1}\right) \geq \overline{\mathrm{E}}_{\mathrm{V}}\left(f \mid x_{1: n+1}\right)$. Fix any $x_{n+1} \in \mathscr{X}$. Then $\mathscr{M}\left(x_{1: n+1}\right)$ is either real or equal to $+\infty$ because $\mathscr{M}$ is bounded below. If $\mathscr{M}\left(x_{1: n+1}\right)$ is real, then since $\mathscr{M}\left(x_{1: n+1}\right) \geq \overline{\mathrm{E}}_{\mathrm{V}}\left(f \mid x_{1: n+1}\right)$, it follows from Definition 3 that there is a bounded below supermartingale $\mathscr{M}_{x_{1: n+1}}$ such that $\mathscr{M}_{x_{1: n+1}}\left(x_{1: n+1}\right) \leq$ $\mathscr{M}\left(x_{1: n+1}\right)+\epsilon$ and liminf $\mathscr{M}_{x_{1: n+1}} \geq_{x_{1: n+1}} f$. If $\mathscr{M}\left(x_{1: n+1}\right)$ is $+\infty$, let $\mathscr{M}_{x_{1: n+1}}$ be the constant supermartingale that is equal to $+\infty$ everywhere. So, for all $x_{n+1} \in \mathscr{X}$, we have found a bounded below supermartingale $\mathscr{M}_{x_{1: n+1}}$ such that $\mathscr{M}_{x_{1: n+1}}\left(x_{1: n+1}\right) \leq \mathscr{M}\left(x_{1: n+1}\right)+\epsilon$ and $\liminf \mathscr{M}_{x_{1: n+1}} \geq_{x_{1: n+1}} f$. Let $\mathscr{M}^{*}$ be the process that is equal to $\mathscr{M}+\epsilon$ for all situations that precede or are incomparable with $x_{1: n}$, and that is equal to $\mathscr{M}_{x_{1: n+1}}$ for all situations that follow $x_{1: n+1}$ for some $x_{n+1} \in \mathscr{X}$. Note that liminf $\mathscr{M}^{*} \geq_{x_{1: n}} f$ because, for each $x_{n+1} \in \mathscr{X}$, we have that $\liminf \mathscr{M}^{*}=_{x_{1: n+1}} \liminf \mathscr{M}_{x_{1: n+1}} \geq_{x_{1: n+1}} f$. We moreover show that $\mathscr{M}^{*}$ is a bounded below supermartingale.

The process $\mathscr{M}^{*}$ is clearly bounded below because $\mathscr{M}$ and all $\mathscr{M}_{x_{1: n+1}}$ are bounded below and $\mathscr{X}$ is finite. Furthermore, for any $x_{n+1} \in \mathscr{X}$, we have that $\mathscr{M}^{*}\left(x_{1: n+1}\right)=\mathscr{M}_{x_{1: n+1}}\left(x_{1: n+1}\right) \leq \mathscr{M}\left(x_{1: n+1}\right)+\epsilon$, implying that $\mathscr{M}^{*}\left(x_{1: n} \cdot\right) \leq$ $\mathscr{M}\left(x_{1: n} \cdot\right)+\epsilon$ and therefore, by[E4 andE8 that

$$
\overline{\mathrm{Q}}_{x_{1: n}}\left(\mathscr{M}^{*}\left(x_{1: n} \cdot\right)\right) \leq \overline{\mathrm{Q}}_{x_{1: n}}\left(\mathscr{M}\left(x_{1: n} \cdot\right)+\epsilon\right)=\overline{\mathrm{Q}}_{x_{1: n}}\left(\mathscr{M}\left(x_{1: n} \cdot\right)\right)+\epsilon \leq \mathscr{M}\left(x_{1: n}\right)+\epsilon=\mathscr{M}^{*}\left(x_{1: n}\right)
$$

Moreover, for all situations $s \nsupseteq x_{1: n}$, we have byE8 that $\overline{\mathrm{Q}}_{s}\left(\mathscr{M}^{*}(s \cdot)\right)=\overline{\mathrm{Q}}_{s}(\mathscr{M}(s \cdot)+\epsilon)=\overline{\mathrm{Q}}_{s}(\mathscr{M}(s \cdot))+\epsilon \leq \mathscr{M}(s)+\epsilon=$ $\mathscr{M}^{*}(s)$, and for all $s \in \mathscr{X}^{*}$ such that $s \sqsupseteq x_{1: n+1}$ for some $x_{n+1} \in \mathscr{X}$, we have that $\overline{\mathrm{Q}}_{s}\left(\mathscr{M}^{*}(s \cdot)\right)=\overline{\mathrm{Q}}_{s}\left(\mathscr{M}_{x_{1: n+1}}(s \cdot)\right) \leq$ $\mathscr{M}_{x_{1: n+1}}(s)=\mathscr{M}^{*}(s)$. All together, we have that $\overline{\mathrm{Q}}_{s}\left(\mathscr{M}^{*}(s \cdot)\right) \leq \mathscr{M}^{*}(s)$ for all $s \in \mathscr{X}^{*}$, implying that $\mathscr{M}^{*}$ is a supermartingale that, as shown before, is bounded below.

Since $\liminf \mathscr{M}^{*} \geq_{x_{1: n}} f$ and $\mathscr{M}^{*}\left(x_{1: n}\right)=\mathscr{M}\left(x_{1: n}\right)+\epsilon \leq \alpha+\epsilon$, Definition 3now implies that $\overline{\mathrm{E}}_{\mathrm{V}}\left(f \mid x_{1: n}\right) \leq \alpha+\epsilon$. This holds for any $\epsilon \in \mathbb{R}_{>0}$ and any real $\alpha>\overline{\mathrm{E}}_{\mathrm{V}}\left(\overline{\mathrm{E}}_{\mathrm{V}}\left(f \mid x_{1: n} X_{n+1}\right) \mid x_{1: n}\right)$, so we indeed conclude that $\overline{\mathrm{E}}_{\mathrm{V}}\left(f \mid x_{1: n}\right) \leq$ $\overline{\mathrm{E}}_{\mathrm{V}}\left(\overline{\mathrm{E}}_{\mathrm{V}}\left(f \mid x_{1: n} X_{n+1}\right) \mid x_{1: n}\right)$.

Corollary 16. For any $f \in \overline{\mathbb{V}}_{b}$, the process $\mathscr{P}$, defined by $\mathscr{P}(s):=\overline{\mathrm{E}}_{\mathrm{V}}(f \mid s)$ for all $s \in \mathscr{X}^{*}$, is a bounded below supermartingale.

Proof. Consider any $f \in \overline{\mathbb{V}}_{b}$. Then $\mathscr{P}$ is bounded below because $f$ is bounded below and $\overline{\mathrm{E}}_{\mathrm{V}}$ satisfies $\mathbf{V 5}$, Moreover, if for any $s \in \mathscr{X}^{*}$ we let $\overline{\mathrm{E}}_{\mathrm{V}}(f \mid s \cdot)$ be the (bounded below) local variable that assumes the value $\overline{\mathrm{E}}_{\mathrm{V}}(f \mid s x)$ for all 
$x \in \mathscr{X}$, then it follows from Proposition[14] and Theorem[15 that

$$
\begin{aligned}
\overline{\mathrm{Q}}_{x_{1: n}}\left(\mathscr{P}\left(x_{1: n} \cdot\right)\right)=\overline{\mathrm{Q}}_{x_{1: n}}\left(\overline{\mathrm{E}}_{\mathrm{V}}\left(f \mid x_{1: n} \cdot\right)\right) & =\overline{\mathrm{E}}_{\mathrm{V}}\left(\overline{\mathrm{E}}_{\mathrm{V}}\left(f \mid x_{1: n} X_{n+1}\right) \mid x_{1: n}\right) \\
& =\overline{\mathrm{E}}_{\mathrm{V}}\left(f \mid x_{1: n}\right)=\mathscr{P}\left(x_{1: n}\right) \text { for all } x_{1: n} \in \mathscr{X}^{*} .
\end{aligned}
$$

Hence, $\mathscr{P}$ is indeed a supermartingale, and therefore a bounded below supermartingale.

\section{Equivalent definitions for $\overline{\mathbf{E}}_{\mathrm{V}}$}

We start the current section by presenting two technical results that are essential for our further analysis of Definition 33. Doob's Convergence Theorem and Lévy's Zero-one Law. Both of them also hold in a precise measure-theoretic context, but our results do not require any measurability conditions, nor do they require the local models to be precise. The game-theoretic versions we present here are due to Shafer, Vovk and Takemura [13, 14, 23]. However, since our framework slightly differs from theirs, we have adapted their proofs to our setting. Some of the involved arguments are rather lengthy and technical, though, so we have chosen to relegate these proofs to an appendix at the end of the paper.

To state the results, we require the following terminology. For any $s \in \mathscr{X}^{*}$, we say that a supermartingale $\mathscr{M} \in \overline{\mathbb{M}}_{\mathrm{b}}$ is an $s$-test supermartingale if it is non-negative and $\mathscr{M}(s)=1$. If $s=\square$, we simply say it is a test supermartingale. For any $s \in \mathscr{X}^{*}$, we say that an event $A \subseteq \Omega$ is strictly almost sure (s.a.s.) within $\Gamma(s)$ if there is an $s$-test supermartingale that converges to $+\infty$ on $\Gamma(s) \backslash A$. In that case, we call the event $A^{c}$ strictly null within $\Gamma(s)$. If $s=\square$, we drop the 'within' and simply speak of 'strictly almost sure'. For any two $f, g \in \overline{\mathbb{V}}$, we will use the notation $f \geq_{s} g$ s.a.s. - and similarly for $\leq_{s},>_{s}$ and $<_{s}$ 一 to indicate that the event $\{\omega \in \Omega: f(\omega) \geq g(\omega)\}$ is strictly almost sure within $\Gamma(s)$.

It can easily be shown that an event $A \subseteq \Omega$ is strictly almost sure within $\Gamma(s)$ if and only $\overline{\mathrm{P}}_{\mathrm{V}}\left(A^{c} \mid s\right)=0$ or, equivalently $\left[3\right.$ if and only if $\underline{\mathrm{P}}_{\mathrm{V}}(A \mid s)=1$; we refer to $\lfloor\underline{13}$, Proposition 8.4] for an illustration in the case where $s=\square$. This is similar to the traditional measure-theoretic definition of an almost sure event; that is, a measurable event with (measure-theoretic) probability one. In contrast with the measure-theoretic definition however, the gametheoretic approach provides a clear behavioural interpretation for strictly null events $A \subseteq \Omega$ : it says that there is a strategy for Skeptic that allows him to start with a finite capital (in the situation $s$ ) and become infinitely rich on all paths $\omega \in A$ (that moreover go through $s$ ) without ever borrowing money.

Theorem 18 below establishes a version of Doob's convergence law. It states that a bounded below supermartingale converges to a real number strictly almost surely. This is somewhat intuitive (yet, not trivial at all): since a supermartingale is bounded below and expected to decrease, one would expect it to converge to a real number. We precede Theorem 18 with a technical result about the limit behaviour of the test supermartingales that are involved in Theorem 18 ,

Proposition 17. Consider any supermartingale $\mathscr{M} \in \overline{\mathbb{M}}_{\mathrm{b}}$. If $\mathscr{M}(s)$ is real for some $s \in \mathscr{X}^{*}$, then there is a s-test supermartingale $\mathscr{M}^{*}$ that converges to $+\infty$ on all paths $\omega \in \Gamma(s)$ where $\mathscr{M}$ does not converge to an extended real number, and that converges to an extended real number on all paths $\omega \in \Gamma(s)$ where $\mathscr{M}$ converges to a real number.

\footnotetext{
${ }^{3}$ This follows from the fact that $\overline{\mathrm{P}}_{\mathrm{V}}\left(A^{c} \mid s\right)=\overline{\mathrm{E}}_{\mathrm{V}}\left(\mathbb{I}_{A^{c}} \mid s\right)=\overline{\mathrm{E}}_{\mathrm{V}}\left(1-\mathbb{I}_{A} \mid s\right)=1+\overline{\mathrm{E}}_{\mathrm{V}}\left(-\mathbb{I}_{A} \mid s\right)=1-\underline{\mathrm{E}}_{\mathrm{V}}\left(\mathbb{I}_{A} \mid s\right)=1-\underline{\mathrm{P}}_{\mathrm{V}}(A \mid s)$, using $\overline{\nabla 6}$ for the third equality.
} 
Theorem 18 (Doob's Convergence Theorem). Consider any supermartingale $\mathscr{M} \in \overline{\mathbb{M}}_{\mathrm{b}}$. If $\mathscr{M}(s)$ is real for some $s \in \mathscr{X}^{*}$, then $\mathscr{M}$ converges to a real number strictly almost surely within $\Gamma(s)$.

The following result, a version of Lévy's zero-one law, captures (and extends) yet another intuitive idea: the upper probability of an event $A \subseteq \Omega$ conditional on a situation $\omega^{n}$ should (or, is expected to) converge to 1 as $n \rightarrow+\infty$ if $\omega \in A$.

Theorem 19 (Lévy's zero-one law). For any $f \in \overline{\mathbb{V}}_{b}$ and any $s \in \mathscr{X}^{*}$, the event

$$
A:=\left\{\omega \in \Omega: \liminf _{n \rightarrow+\infty} \overline{\mathrm{E}}_{\mathrm{V}}\left(f \mid \omega^{n}\right) \geq f(\omega)\right\} \text { is strictly almost sure within } \Gamma(s) .
$$

One of the major consequences of Doob's convergence theorem and Lévy's zero-one law is that they allow us to draw some interesting conclusions about Definition 3 In particular, we can use them to show that the resulting game-theoretic upper expectation is not impacted much by changes that concern the limit behaviour of supermartingales and, more specifically, how this limit behaviour relates to the variable $f$ at hand; see Proposition20 and21 below. As was the case for the previous results in this section, the ideas underlying the proofs of the following results are due to Shafer, Vovk and Takemura [13, 14].

Our first result shows that, in Definition 3 we can restrict ourselves to the bounded below supermartingales that converge within $\Gamma(s)$. That is, the limit inferior in Definition 3 can be replaced by a limit.

Proposition 20. For any $f \in \overline{\mathbb{V}}$ and any $s \in \mathscr{X}^{*}$, we have that

$$
\overline{\mathrm{E}}_{\mathrm{V}}(f \mid s)=\inf \left\{\mathscr{M}(s): \mathscr{M} \in \overline{\mathbb{M}}_{\mathrm{b}} \text { and } \lim \mathscr{M} \geq_{s} f\right\},
$$

where the condition $\lim \mathscr{M} \geq_{s} f$ is taken to implicitly imply that $\lim \mathscr{M}$ exists within $\Gamma(s)$.

Proof. The inequality ‘ $\leq$ ' is trivially satisfied since $\liminf \mathscr{M}={ }_{s} \lim \mathscr{M}$ for any bounded below supermartingale $\mathscr{M}$ such that the $\operatorname{limit} \lim \mathscr{M}$ exists within $\Gamma(s)$. It remains to prove the other inequality. If $\overline{\mathrm{E}}_{\mathrm{V}}(f \mid s)=+\infty$, this is trivially satisfied. Otherwise, fix any real $\alpha>\overline{\mathrm{E}}_{\mathrm{V}}(f \mid s)$. Then, due to Definition 3 there is a supermartingale $\mathscr{M} \in \overline{\mathbb{M}}_{\mathrm{b}}$ such that $\mathscr{M}(s) \leq \alpha$ and $\liminf \mathscr{M} \geq_{s} f$. Because $\mathscr{M}$ is bounded below and $\alpha$ is real, $\mathscr{M}(s)$ is also real. So, by Proposition 17 there is an $s$-test supermartingale $\mathscr{M}^{*}$ that converges to $+\infty$ on all paths $\omega \in \Gamma(s)$ where $\mathscr{M}$ does not converge in $\overline{\mathbb{R}}$ and converges in $\overline{\mathbb{R}}$ on all paths $\omega \in \Gamma(s)$ where $\mathscr{M}$ converges in $\mathbb{R}$.

Fix any $\epsilon \in \mathbb{R}_{>0}$ and consider the process $\mathscr{M}^{\prime}$ defined by $\mathscr{M}^{\prime}(t):=\mathscr{M}(t)+\epsilon \mathscr{M}^{*}(t)$ for all situations $t \sqsupseteq s$ and by $\mathscr{M}^{\prime}(t):=\mathscr{M}(s)+\epsilon \mathscr{M}^{*}(s) \leq \alpha+\epsilon$ for all situations $t \nsupseteq s$. Then $\mathscr{M}^{\prime}$ is bounded below because $\mathscr{M}$ and $\mathscr{M}^{*}$ are bounded below. Moreover, as we will now show, it is also a supermartingale. On the one hand, for all situations $t \sqsupseteq s$, we have that

$$
\overline{\mathrm{Q}}_{t}\left(\mathscr{M}^{\prime}(t \cdot)\right)=\overline{\mathrm{Q}}_{t}\left(\mathscr{M}(t \cdot)+\epsilon \mathscr{M}^{*}(t \cdot)\right) \stackrel{\mathrm{E} 2[\mathrm{E3}}{\leq}-\overline{\mathrm{Q}}_{t}(\mathscr{M}(t \cdot))+\epsilon \overline{\mathrm{Q}}_{t}\left(\mathscr{M}^{*}(t \cdot)\right) \leq \mathscr{M}(t)+\epsilon \mathscr{M}^{*}(t)=\mathscr{M}^{\prime}(t),
$$

where the second inequality follows from the fact that $\mathscr{M}$ and $\mathscr{M}^{*}$ are bounded below supermartingales, and from the positivity of $\epsilon$. On the other hand, for all situations $t \nsupseteq s$, it can easily be seen that the local variable $\mathscr{M}^{\prime}(t)$ is equal to the constant $\mathscr{M}(s)+\epsilon \mathscr{M}^{*}(s)=\mathscr{M}^{\prime}(t)$. This constant is furthermore real because $\mathscr{M}^{\prime}(t)$ is bounded below and $\mathscr{M}(s)+\epsilon \mathscr{M}^{*}(s) \leq \alpha+\epsilon$. Hence, due to E1] $\overline{\mathrm{Q}}_{t}\left(\mathscr{M}^{\prime}(t \cdot)\right)=\mathscr{M}^{\prime}(t)$. So we can conclude that $\mathscr{M}^{\prime}$ is indeed a supermartingale and more specifically, a bounded below supermartingale. We also have that 
$\liminf \mathscr{M}^{\prime} \geq_{s} f$ because $\epsilon \mathscr{M}^{*}$ is non-negative and liminf $\mathscr{M} \geq_{s} f$. We will now show that, moreover, for all $\omega \in \Gamma(s)$, this process $\mathscr{M}^{\prime}$ converges in $\overline{\mathbb{R}}$.

For any $\omega \in \Gamma(s)$, if $\mathscr{M}$ does not converge in $\overline{\mathbb{R}}, \mathscr{M}^{*}$ converges to $+\infty$ and hence also $\mathscr{M}^{\prime}$ because $\mathscr{M}$ is bounded below and $\epsilon$ is positive. If $\mathscr{M}$ does converge in $\overline{\mathbb{R}}$, it converges either to a real number or to $+\infty$ (convergence to $-\infty$ is impossible because it is bounded below). If $\mathscr{M}$ converges to a real number, $\mathscr{M}^{*}$ converges in $\overline{\mathbb{R}}$ and hence $\mathscr{M}^{\prime}$ also converges in $\overline{\mathbb{R}}$. If $\mathscr{M}$ converges to $+\infty$, then so does $\mathscr{M}^{\prime}$ because $\epsilon \mathscr{M}^{*}$ is non-negative. Hence, for all $\omega \in \Gamma(s), \mathscr{M}^{\prime}$ converges in $\overline{\mathbb{R}}$ and the limit $\lim \mathscr{M}^{\prime}(\omega)$ therefore exists.

Now, recall that $\lim \mathscr{M}^{\prime}=\liminf \mathscr{M}^{\prime} \geq_{s} f$ and that $\mathscr{M} \in \overline{\mathbb{M}}_{\mathrm{b}}$. Hence, we have that

$$
\inf \left\{\mathscr{M}(s): \mathscr{M} \in \overline{\mathbb{M}}_{\mathrm{b}} \text { and } \lim \mathscr{M} \geq_{s} f\right\} \leq \mathscr{M}^{\prime}(s)=\mathscr{M}(s)+\epsilon \mathscr{M}^{*} \leq \alpha+\epsilon .
$$

This holds for any $\epsilon \in \mathbb{R}_{>0}$ and any $\alpha>\overline{\mathrm{E}}_{\mathrm{V}}(f \mid s)$, which implies that indeed

$$
\inf \left\{\mathscr{M}(s): \mathscr{M} \in \overline{\mathbb{M}}_{\mathrm{b}} \text { and } \lim \mathscr{M} \geq_{s} f\right\} \leq \overline{\mathrm{E}}_{\mathrm{V}}(f \mid s) .
$$

The following result shows that the condition $\liminf \mathscr{M} \geq_{s} f$ in Definition 3 should in fact merely hold strictly almost surely:

Proposition 21. Consider any $f \in \overline{\mathbb{V}}$ and any $s \in \mathscr{X}^{*}$. Then

$$
\overline{\mathrm{E}}_{\mathrm{V}}(f \mid s)=\inf \left\{\mathscr{M}(s): \mathscr{M} \in \overline{\mathbb{M}}_{\mathrm{b}} \text { and } \liminf \mathscr{M} \geq_{\text {s }} f \text { s.a.s. }\right\} .
$$

Proof. Since every supermartingale $\mathscr{M}$ that satisfies $\liminf \mathscr{M} \geq_{s} f$ also satisfies $\liminf \mathscr{M} \geq_{s} f$ s.a.s., we clearly have that

$$
\overline{\mathrm{E}}_{\mathrm{V}}(f \mid s) \geq \inf \left\{\mathscr{M}(s): \mathscr{M} \in \overline{\mathbb{M}}_{\mathrm{b}} \text { and } \liminf \mathscr{M} \geq_{s} f \text { s.a.s. }\right\},
$$

so it remains to prove the other inequality. If the right hand side of Equation (6) is equal to $+\infty$, then this inequality is trivially satisfied. So consider the case where it is not. Fix any $\alpha \in \mathbb{R}$ such that $\alpha>\inf \{\mathscr{M}(s): \mathscr{M} \in$ $\overline{\mathbb{M}}_{\mathrm{b}}$ and liminf $\mathscr{M} \geq_{s} f$ s.a.s. $\}$ and any $\epsilon \in \mathbb{R}_{>0}$. Then there is some bounded below supermartingale $\mathscr{M}_{\alpha}$ such that $\liminf \mathscr{M}_{\alpha} \geq_{s} f$ s.a.s. and

$$
\mathscr{M}_{\alpha}(s) \leq \alpha .
$$

Since $\liminf \mathscr{M}_{\alpha} \geq_{s} f$ s.a.s., there is some $s$-test supermartingale $\mathscr{M}_{\alpha}^{*}$ that converges to $+\infty$ on $A:=\{\omega \in$ $\Gamma(s)$ : $\left.\liminf \mathscr{M}_{\alpha}(\omega)<f(\omega)\right\}$. Consider the extended real process $\mathscr{M}_{\alpha}+\epsilon \mathscr{M}_{\alpha}^{*}$. This process is again a bounded below supermartingale because of Lemma [2] [which we can apply because $\mathscr{M}_{\alpha}$ and $\mathscr{M}_{\alpha}^{*}$ are both bounded below and hence have a common lower bound]. Since $\mathscr{M}_{\alpha}^{*}$ converges to $+\infty$ on $A$ and because $\mathscr{M}_{\alpha}$ is bounded below, we have that $\liminf \left(\mathscr{M}_{\alpha}+\epsilon \mathscr{M}_{\alpha}^{*}\right)(\omega)=+\infty \geq f(\omega)$ for all $\omega \in A$. Moreover, for all $\omega \in \Gamma(s) \backslash A$, we also have that $\liminf \left(\mathscr{M}_{\alpha}+\epsilon \mathscr{M}_{\alpha}^{*}\right)(\omega) \geq f(\omega)$, because liminf $\mathscr{M}_{\alpha}(\omega) \geq f(\omega)$ and because $\epsilon \mathscr{M}_{\alpha}^{*}$ is non-negative. Hence, $\liminf \left(\mathscr{M}_{\alpha}+\epsilon \mathscr{M}_{\alpha}^{*}\right) \geq_{s} f$ and consequently $\overline{\mathrm{E}}_{\mathrm{V}}(f \mid s) \leq\left(\mathscr{M}_{\alpha}+\epsilon \mathscr{M}_{\alpha}^{*}\right)(s)$. It therefore follows from Equation (7) that

$$
\overline{\mathrm{E}}_{\mathrm{V}}(f \mid s) \leq\left(\mathscr{M}_{\alpha}+\epsilon \mathscr{M}_{\alpha}^{*}\right)(s)=\mathscr{M}_{\alpha}(s)+\epsilon \leq \alpha+\epsilon .
$$

As this holds for any $\epsilon \in \mathbb{R}_{>0}$, we have that $\overline{\mathrm{E}}_{\mathrm{V}}(f \mid s) \leq \alpha$, and since this is true for every $\alpha \in \mathbb{R}$ such that $\alpha>$ $\inf \left\{\mathscr{M}(s): \mathscr{M} \in \overline{\mathbb{M}}_{\mathrm{b}}\right.$ and $\liminf \mathscr{M} \geq_{s} f$ s.a.s. $\}$, it follows that

$$
\overline{\mathrm{E}}_{\mathrm{V}}(f \mid s) \leq \inf \left\{\mathscr{M}(s): \mathscr{M} \in \overline{\mathbb{M}}_{\mathrm{b}} \text { and } \liminf \mathscr{M} \geq_{s} f \text { s.a.s. }\right\} .
$$


Clearly, the infimum in (6) is taken over a larger set compared to the infimum in Definition3. Though the resulting game-theoretic upper expectation is not impacted by this difference, it does make sure that the infimum in [6] becomes attained:

Proposition 22. For any $f \in \overline{\mathbb{V}}_{b}$ and any $s \in \mathscr{X}^{*}$, the infimum in Equation [6] is attained.

Proof. Consider any $f \in \overline{\mathbb{V}}_{b}$ and any $s \in \mathscr{X}^{*}$. Let $\mathscr{P}$ be the extended real process defined by $\mathscr{P}(t):=\overline{\mathrm{E}}_{\mathrm{V}}(f \mid t)$ for all $t \in \mathscr{X}^{*}$. Then $\mathscr{P}$ is a bounded below supermartingale because of Corollary[16, Moreover, because of Theorem 19. we have that $\liminf \mathscr{P} \geq_{s} f$ strictly almost surely. Since $\mathscr{P}(s)=\overline{\mathrm{E}}_{\mathrm{V}}(f \mid s)$, this concludes the proof.

\section{Continuity of $\bar{E}_{V}$ with respect to monotone sequences}

We now turn to the most important subject in this paper: continuity properties of $\overline{\mathrm{E}}_{\mathrm{V}}$. Apart from their intrinsic theoretical value, these properties also have great practical relevance, in the sense that they provide possible ways to calculate (upper) expectations that would otherwise be difficult or even impossible to calculate numerically. For example, suppose that we have some variable $f \in \overline{\mathbb{V}}$ and some situation $s \in \mathscr{X}^{*}$ for which calculating $\overline{\mathrm{E}}_{\mathrm{V}}(f \mid s)$ directly is not feasible. If we can find a sequence of simpler functions $f_{n}$ that converges to $f$ in such a way that $\overline{\mathrm{E}}_{\mathrm{V}}$ is continuous with respect to this convergence, then we can use $\overline{\mathrm{E}}_{\mathrm{V}}\left(f_{n} \mid s\right)$ to approximate $\overline{\mathrm{E}}_{\mathrm{V}}(f \mid s)$ provided that $n$ is large enough. If the sequence of functions $f_{n}$ is moreover simple enough such that all individual $\overline{\mathrm{E}}_{\mathrm{V}}\left(f_{n} \mid s\right)$ can be calculated directly, then we obtain a practical method for calculating $\overline{\mathrm{E}}_{\mathrm{V}}(f \mid s)$.

We start by establishing results similar to those presented in an earlier conference paper [20], where we used a version of $\overline{\mathrm{E}}_{\mathrm{V}}$ with real supermartingales instead of extended real ones. These results mainly concern continuity with respect to monotone sequences that are bounded below. The first one shows that, similar to the local models $\overline{\mathrm{Q}}_{s}$, the global upper expectation $\overline{\mathrm{E}}_{\mathrm{V}}$ also satisfies continuity with respect to non-decreasing sequences that are bounded below. The idea behind this result goes back to [23, Theorem 6.6], but an updated version can now also be found in $\lfloor 13$, Proposition 8.3]. Once more, the setting for which \13, Proposition 8.3] is stated slightly differs from ours; the authors do not necessarily consider a finite state space, and we do not impose the additional axioms E2'E4' on the local models. Moreover, they only give an explicit proof for the case that there is a single, fixed local model $\bar{Q}$ in all situations. For these reasons, we provide an independent proof here.

Theorem 23. Consider any $s \in \mathscr{X}^{*}$ and any non-decreasing sequence $\left\{f_{n}\right\}_{n \in \mathbb{N}_{0}}$ in $\overline{\mathbb{V}}_{b}$ that converges pointwise to a variable $f \in \overline{\mathbb{V}}_{b}$. Then we have that $\overline{\mathrm{E}}_{\mathrm{V}}(f \mid s)=\lim _{n \rightarrow+\infty} \overline{\mathrm{E}}_{\mathrm{V}}\left(f_{n} \mid s\right)$.

Proof. As $f_{0} \in \overline{\mathbb{V}}_{b}$ is bounded below and the sequence $\left\{f_{n}\right\}_{n \in \mathbb{N}_{0}}$ is non-decreasing, there is an $M \in \mathbb{R}$ such that $f_{n} \geq M$ for all $n \in \mathbb{N}_{0}$ and therefore, $f$ is also bounded below by $M$. Hence, since $\overline{\mathrm{E}}_{\mathrm{V}}$ is constant additive [V6], we can assume without loss of generality that $f$ and all $f_{n}$ are non-negative.

That $\lim _{n \rightarrow+\infty} \overline{\mathrm{E}}_{\mathrm{V}}\left(f_{n} \mid s\right)$ exists, follows from the non-decreasing character of $\left\{f_{n}\right\}_{n \in \mathbb{N}_{0}}$ and $\overline{\nabla 4}$, Moreover, we have that $\overline{\mathrm{E}}_{\mathrm{V}}(f \mid s) \geq \lim _{n \rightarrow+\infty} \overline{\mathrm{E}}_{\mathrm{V}}\left(f_{n} \mid s\right)$ because $f \geq f_{n}$ [since $\left\{f_{n}\right\}_{n \in \mathbb{N}_{0}}$ is non-decreasing] and because $\overline{\mathrm{E}}_{\mathrm{V}}$ satisfies $\nabla 4$, It remains to prove the converse inequality.

For any $n \in \mathbb{N}_{0}$, consider the extended real process $S_{n}$, defined by $S_{n}(t):=\overline{\mathrm{E}}_{\mathrm{V}}\left(f_{n} \mid t\right)$ for all $t \in \mathscr{X}^{*}$ and the extended real process $S$ defined by the limit $S(t):=\lim _{n \rightarrow+\infty} S_{n}(t)$ for all $t \in \mathscr{X}^{*}$. This limit exists because $\left\{S_{n}(t)\right\}_{n \in \mathbb{N}_{0}}$ 
is a non-decreasing sequence for all $t \in \mathscr{X}^{*}$, due to the monotonicity [V4] of $\overline{\mathrm{E}}_{\mathrm{V}}$. As $f_{n}$ is non-negative for all $n \in \mathbb{N}_{0}, S_{n}$ is non-negative for all $n \in \mathbb{N}_{0}$ because of $\mathbf{V 5}$ and therefore $S$ is also non-negative. As a result, $S$ and all $S_{n}$ are non-negative extended real processes.

It now suffices to prove that $S$ is a bounded below supermartingale such that $\liminf S \geq_{s} f$ s.a.s. because it will then follow from Proposition21 that

$$
\begin{aligned}
\overline{\mathrm{E}}_{\mathrm{V}}(f \mid s) & =\inf \left\{\mathscr{M}(s): \mathscr{M} \in \overline{\mathbb{M}}_{\mathrm{b}} \text { and liminf } \mathscr{M} \geq_{s} f \text { s.a.s. }\right\} \\
& \leq S(s)=\lim _{n \rightarrow+\infty} \overline{\mathrm{E}}_{\mathrm{V}}\left(f_{n} \mid s\right) .
\end{aligned}
$$

This is what we now set out to do.

We first show that $S$ is a supermartingale; that it is bounded below follows trivially from its non-negativity. For all situations $t \in \mathscr{X}^{*}$, we already know that $\left\{S_{n}(t \cdot)\right\}_{n \in \mathbb{N}_{0}}$ is a non-decreasing sequence that converges to $S(t \cdot)$. Since $S_{n}$ and $S$ are non-negative, we also have that $S_{n}(t \cdot), S(t \cdot) \in \overline{\mathscr{L}}_{b}(\mathscr{X})$. Then, due toE12, we have that

$$
\overline{\mathrm{Q}}_{t}(S(t \cdot))=\lim _{n \rightarrow+\infty} \overline{\mathrm{Q}}_{t}\left(S_{n}(t \cdot)\right) \text { for all } t \in \mathscr{X}^{*} .
$$

$S_{n}$ is a supermartingale for all $n \in \mathbb{N}_{0}$ because of Corollary 16 so it follows that $\overline{\mathrm{Q}}_{t}\left(S_{n}(t \cdot)\right) \leq S_{n}(t)$ for all $n \in \mathbb{N}_{0}$ and all $t \in \mathscr{X}^{*}$. This implies, together with Equation [8, that

$$
\overline{\mathrm{Q}}_{t}(S(t \cdot)) \leq \lim _{n \rightarrow+\infty} S_{n}(t)=S(t) \text { for all } t \in \mathscr{X}^{*} .
$$

Hence, $S$ is a supermartingale.

To prove that $\liminf S \geq_{s} f$ s.a.s., we will use Lévy's zero-one law. It follows from Theorem 19 that, for all $n \in \mathbb{N}_{0}$, there is an $s$-test supermartingale $\mathscr{M}_{n}$ that converges to $+\infty$ on the event

$$
A_{n}:=\left\{\omega \in \Gamma(s): \liminf _{m \rightarrow+\infty} \overline{\mathrm{E}}_{\mathrm{V}}\left(f_{n} \mid \omega^{m}\right)<f_{n}(\omega)\right\} .
$$

Now, consider the extended real process $\mathscr{M}$, defined by

$$
\mathscr{M}(t):=\sum_{n \in \mathbb{N}_{0}} \lambda_{n} \mathscr{M}_{n}(t) \text { for all } t \in \mathscr{X}^{*},
$$

where the coefficients $\lambda_{n}>0$ sum to 1 . Then it follows from Lemma12 that $\mathscr{M}$ is again a non-negative supermartingale. Moreover, it is clear that $\mathscr{M}(s)=1$ and hence, $\mathscr{M}$ is an $s$-test supermartingale.

We show that $\mathscr{M}$ converges to $+\infty$ on all paths $\omega \in \Gamma(s)$ such that $\liminf _{m \rightarrow+\infty} S\left(\omega^{m}\right)<f(\omega)$. Clearly, $\mathscr{M}$ converges to $+\infty$ on $\cup_{n \in \mathbb{N}_{0}} A_{n}=: A$. Consider now any path $\omega \in \Gamma(s)$ for which $\liminf _{m \rightarrow+\infty} S\left(\omega^{m}\right)<f(\omega)$. As explained before, $S_{n}(t)$ is non-decreasing in $n$ for all $t \in \mathscr{X}^{*}$, so we have that $\sup _{n \in \mathbb{N}_{0}} S_{n}\left(\omega^{m}\right)=\lim _{n \rightarrow+\infty} S_{n}\left(\omega^{m}\right)=$ $S\left(\omega^{m}\right)$ for all $m \in \mathbb{N}_{0}$. Since $\liminf _{m \rightarrow+\infty} S\left(\omega^{m}\right)<f(\omega)$, this implies that

$$
\liminf _{m \rightarrow+\infty} \sup _{n \in \mathbb{N}_{0}} S_{n}\left(\omega^{m}\right)<\lim _{n \rightarrow+\infty} f_{n}(\omega) .
$$

Since $\sup _{n \in \mathbb{N}_{0}} \liminf _{m \rightarrow+\infty} S_{n}\left(\omega^{m}\right) \leq \liminf _{m \rightarrow+\infty} \sup _{n \in \mathbb{N}_{0}} S_{n}\left(\omega^{m}\right)$ [because we obviously have that $S_{n}\left(\omega^{m}\right) \leq$ $\sup _{n \in \mathbb{N}_{0}} S_{n}\left(\omega^{m}\right)$ for all $\left.n, m \in \mathbb{N}_{0}\right]$, this implies that

$$
\sup _{n \in \mathbb{N}_{0}} \liminf _{m \rightarrow+\infty} \overline{\mathrm{E}}_{\mathrm{V}}\left(f_{n} \mid \omega^{m}\right)=\sup _{n \in \mathbb{N}_{0}} \liminf _{m \rightarrow+\infty} S_{n}\left(\omega^{m}\right) \leq \liminf _{m \rightarrow+\infty} \sup _{n \in \mathbb{N}_{0}} S_{n}\left(\omega^{m}\right)<\lim _{n \rightarrow+\infty} f_{n}(\omega) .
$$


Hence, there is some $n_{\omega} \in \mathbb{N}_{0}$ such that

$$
\sup _{n \in \mathbb{N}_{0}} \liminf _{m \rightarrow+\infty} \overline{\mathrm{E}}_{\mathrm{V}}\left(f_{n} \mid \omega^{m}\right)<f_{n_{\omega}}(\omega)
$$

and therefore, we see that also

$$
\liminf _{m \rightarrow+\infty} \overline{\mathrm{E}}_{\mathrm{V}}\left(f_{n_{\omega}} \mid \omega^{m}\right)<f_{n_{\omega}}(\omega)
$$

So $\omega \in A_{n_{\omega}} \subseteq A$ and, as a consequence, $\mathscr{M}$ converges to $+\infty$ on $\omega$. Hence, the $s$-test supermartingale $\mathscr{M}$ converges to $+\infty$ on all paths $\omega \in \Gamma(s)$ such that $\liminf _{m \rightarrow+\infty} S\left(\omega^{m}\right)<f(\omega)$, and therefore $\liminf S \geq_{s} f$ strictly almost surely.

The fact that $\overline{\mathrm{E}}_{\mathrm{V}}$ satisfies continuity with respect to non-decreasing sequences, together with the properties in Proposition 13 immediately implies that $\overline{\mathrm{E}}_{\mathrm{V}}$ is an upper expectation.

Corollary 24. For any $s \in \mathscr{X}^{*}$, the map $\overline{\mathrm{E}}_{\mathrm{V}}(\cdot \mid s): \overline{\mathbb{V}} \rightarrow \overline{\mathbb{R}}$ is an upper expectation on $\overline{\mathbb{V}}$.

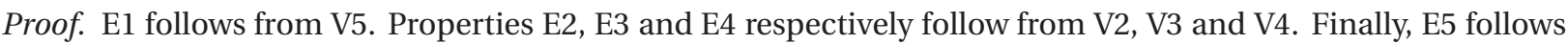
from Theorem 23.

Another immediate consequence of Theorem 23 is that $\overline{\mathrm{E}}_{\mathrm{V}}$ satisfies a version of Fatou's lemma:

Corollary 25 (Fatou's Lemma). For any situation $s \in \mathscr{X}^{*}$ and any sequence $\left\{f_{n}\right\}_{n \in \mathbb{N}_{0}}$ in $\overline{\mathbb{V}}_{b}$ that is uniformly bounded below, we have that $\overline{\mathrm{E}}_{\mathrm{V}}(f \mid s) \leq \liminf _{n \rightarrow+\infty} \overline{\mathrm{E}}_{\mathrm{V}}\left(f_{n} \mid s\right)$ where $f:=\liminf _{n \rightarrow+\infty} f_{n}$.

Proof. Consider any $s \in \mathscr{X}^{*}$ and any sequence $\left\{f_{n}\right\}_{n \in \mathbb{N}_{0}}$ in $\overline{\mathbb{V}}_{b}$ that is uniformly bounded below. For all $k \in \mathbb{N}_{0}$, let $g_{k}$ be the global variable defined by $g_{k}(\omega):=\inf _{n \geq k} f_{n}(\omega)$ for all $\omega \in \Omega$. Then $\lim _{k \rightarrow+\infty} g_{k}=\liminf _{n \rightarrow+\infty} f_{n}=$ : $f$. Furthermore, $\left\{g_{k}\right\}_{k \in \mathbb{N}_{0}}$ is clearly non-decreasing and it is a sequence in $\overline{\mathbb{V}}_{b}$ because $\left\{f_{n}\right\}_{n \in \mathbb{N}_{0}}$ is uniformly bounded below. Hence, we can use Theorem 23 to find that

$$
\overline{\mathrm{E}}_{\mathrm{V}}(f \mid s)=\lim _{k \rightarrow+\infty} \overline{\mathrm{E}}_{\mathrm{V}}\left(g_{k} \mid s\right)=\liminf _{k \rightarrow+\infty} \overline{\mathrm{E}}_{\mathrm{V}}\left(g_{k} \mid s\right) \leq \liminf _{k \rightarrow+\infty} \overline{\mathrm{E}}_{\mathrm{V}}\left(f_{k} \mid s\right),
$$

where the inequality holds because, for all $k \in \mathbb{N}_{0}, g_{k} \leq f_{k}$ and therefore, because of $\overline{\mathrm{V} 4}$ also $\overline{\mathrm{E}}_{\mathrm{V}}\left(g_{k} \mid s\right) \leq \overline{\mathrm{E}}_{\mathrm{V}}\left(f_{k} \mid s\right)$.

The following result is entirely new and states that $\overline{\mathrm{E}}_{\mathrm{V}}$ is continuous with respect to non-increasing sequences of lower cuts. So $\overline{\mathrm{E}}_{\mathrm{V}}(\cdot \mid s)$ satisfies[E6 on $\overline{\mathbb{V}}$ for any $s \in \mathscr{X}^{*}$.

Proposition 26. For any $f \in \overline{\mathbb{V}}$ and any $s \in \mathscr{X}^{*}$, we have that $\lim _{\alpha \rightarrow-\infty} \overline{\mathrm{E}}_{\mathrm{V}}\left(f^{\vee \alpha} \mid s\right)=\overline{\mathrm{E}}_{\mathrm{V}}(f \mid s)$.

Proof. $\overline{\mathrm{E}}_{\mathrm{V}}\left(f^{\vee \alpha} \mid s\right)$ is non-decreasing in $\alpha$ because $f^{\vee \alpha}$ is non-decreasing in $\alpha$ and because $\overline{\mathrm{E}}_{\mathrm{V}}$ is monotone [V4, and therefore $\lim _{\alpha \rightarrow-\infty} \overline{\mathrm{E}}_{\mathrm{V}}\left(f^{\vee \alpha} \mid s\right)$ exists. Moreover, $f^{\vee \alpha} \geq f$ for all $\alpha \in \mathbb{R}$, implying, by the monotonicity $\mathbb{\mathrm { V } 4}$ of $\overline{\mathrm{E}}_{\mathrm{V}}$, that $\lim _{\alpha \rightarrow-\infty} \overline{\mathrm{E}}_{\mathrm{V}}\left(f^{\vee \alpha} \mid s\right) \geq \overline{\mathrm{E}}_{\mathrm{V}}(f \mid s)$. It therefore only remains to prove the converse inequality.

If $\overline{\mathrm{E}}_{\mathrm{V}}(f \mid s)=+\infty$, then $\lim _{\alpha \rightarrow-\infty} \overline{\mathrm{E}}_{\mathrm{V}}\left(f^{\vee \alpha} \mid s\right) \leq \overline{\mathrm{E}}_{\mathrm{V}}(f \mid s)$ holds trivially. If $\overline{\mathrm{E}}_{\mathrm{V}}(f \mid s)<+\infty$, fix any real $c>\overline{\mathrm{E}}_{\mathrm{V}}(f \mid s)$. Then it follows from the definition of $\overline{\mathrm{E}}_{\mathrm{V}}(f \mid s)$ that there is some supermartingale $\mathscr{M} \in \overline{\mathbb{M}}_{\mathrm{b}}$ such that $\mathscr{M}(s) \leq c$ and liminf $\mathscr{M} \geq_{s} f$. Since $\mathscr{M}$ is bounded below, it immediately follows that there is some $B \in \mathbb{R}$ such that 
$\liminf \mathscr{M} \geq \alpha$ for all $\alpha \leq B$. For such an $\alpha \leq B$, we have that $\liminf \mathscr{M} \geq_{s} f^{\vee \alpha}$, which by Definitionßimplies that $\overline{\mathrm{E}}_{\mathrm{V}}\left(f^{\vee \alpha} \mid s\right) \leq \mathscr{M}(s) \leq c$. This holds for all $\alpha \leq B$, so we infer that $\lim _{\alpha \rightarrow-\infty} \overline{\mathrm{E}}_{\mathrm{V}}\left(f^{\vee \alpha} \mid s\right) \leq c$, and since this holds for any $c>\overline{\mathrm{E}}_{\mathrm{V}}(f \mid s)$, we conclude that indeed $\lim _{\alpha \rightarrow-\infty} \overline{\mathrm{E}}_{\mathrm{V}}\left(f^{\vee \alpha} \mid s\right) \leq \overline{\mathrm{E}}_{\mathrm{V}}(f \mid s)$.

Proposition 26] shows that $\overline{\mathrm{E}}_{\mathrm{V}}$ on $\overline{\mathbb{V}} \times \mathscr{X}^{*}$ is uniquely determined by its values on $\overline{\mathbb{V}}_{b} \times \mathscr{X}^{*}$. Moreover, by Theorem 23 its values on $\overline{\mathbb{V}}_{b} \times \mathscr{X}^{*}$ are on their turn uniquely determined by its values on $\mathbb{V} \times \mathscr{X}^{*}$ (for any $f \in \overline{\mathbb{V}}_{b}$, there obviously is at least one sequence in $\mathbb{V}$ that converges non-decreasingly to $f$ ). Together, these observations imply that $\overline{\mathrm{E}}_{\mathrm{V}}$ is uniquely determined by its values on the gambles in $\overline{\mathbb{V}}$.

Proposition26also immediately establishes our claim from Section2, that the (global) game-theoretic upper expectation is only fully compatible with the local models if the local models additionally satisfy E6

Corollary 27 (Compatibility with local models). For any imprecise probabilities tree $\overline{\mathrm{Q}}$, we have that $\overline{\mathrm{E}}_{\mathrm{V}}\left(f \mid x_{1: n}\right)=$ $\overline{\mathrm{Q}}_{x_{1: n}}\left(f\left(x_{1: n} \cdot\right)\right)$ for all $x_{1: n} \in \mathscr{X}^{*}$ and all $(n+1)$-measurable variables $f \in \overline{\mathbb{V}}$, if and only if the local upper expectations $\overline{\mathrm{Q}}_{\text {s }}$ all satisfy $\mathrm{E6}$ on $\overline{\mathscr{L}}(\mathscr{X})$.

Proof. Consider any $x_{1: n} \in \mathscr{X}^{*}$ and any $(n+1)$-measurable extended real variable $f \in \overline{\mathbb{V}}$. Clearly, $f^{\vee c}$ is bounded below and remains to be $(n+1)$-measurable for any $c \in \mathbb{R}$. Due to Proposition[14 we have that $\overline{\mathrm{E}}_{\mathrm{V}}\left(f^{\vee c} \mid x_{1: n}\right)=$ $\overline{\mathrm{Q}}_{x_{1: n}}\left(f^{\vee c}\left(x_{1: n} \cdot\right)\right)$ for any $c \in \mathbb{R}$. Then, because $\overline{\mathrm{E}}_{\mathrm{V}}\left(\cdot \mid x_{1: n}\right)$ satisfies[E6 due to Proposition 26 we clearly also have that $\overline{\mathrm{E}}_{\mathrm{V}}\left(f \mid x_{1: n}\right)=\overline{\mathrm{Q}}_{x_{1: n}}\left(f\left(x_{1: n} \cdot\right)\right)$ if $\overline{\mathrm{Q}}_{x_{1: n}}$ satisfiesE6

On the other hand, consider the case that there is some $x_{1: n} \in \mathscr{X}^{*}$ such that $\overline{\mathrm{Q}}_{x_{1: n}}$ does not satisfyE6 meaning that there is at least a single $f \in \overline{\mathscr{L}}(\mathscr{X})$ such that $\overline{\mathrm{Q}}_{x_{1: n}}(f) \neq \lim _{c \rightarrow-\infty} \overline{\mathrm{Q}}_{x_{1: n}}\left(f^{\vee c}\right)$ [the limit on the right hand side still exists because of monotonicity [E4)]. Let $g \in \overline{\mathbb{V}}$ be an $(n+1)$-measurable variable such that $g\left(x_{1: n} \cdot\right)=f$. Then, for all $c \in \mathbb{R}, g^{\vee c}$ is bounded below and $(n+1)$-measurable and we clearly also have that $g^{\vee c}\left(x_{1: n} \cdot\right)=f^{\vee c}$. Hence,

$$
\overline{\mathrm{Q}}_{x_{1: n}}\left(g\left(x_{1: n} \cdot\right)\right)=\overline{\mathrm{Q}}_{x_{1: n}}(f) \neq \lim _{c \rightarrow-\infty} \overline{\mathrm{Q}}_{x_{1: n}}\left(f^{\vee c}\right)=\lim _{c \rightarrow-\infty} \overline{\mathrm{Q}}_{x_{1: n}}\left(g^{\vee c}\left(x_{1: n} \cdot\right)\right)=\lim _{c \rightarrow-\infty} \overline{\mathrm{E}}_{\mathrm{V}}\left(g^{\vee c} \mid x_{1: n}\right)=\overline{\mathrm{E}}_{\mathrm{V}}\left(g \mid x_{1: n}\right),
$$

where the second to last step follows from Proposition 14 and the last step follows from Proposition 26.

As we have illustrated in Example 1 an upper expectation - and therefore also any local upper expectationdoes not necessarily satisfyE6 Hence, if we find compatibility (on the entire domain) between local and global models desirable, then we will need to impose E6] as an additional axiom on the local models $\overline{\mathrm{Q}}_{s}$. This is also the case even when we adopt the stronger axioms E2' E4' (again, this follows from Example@. We therefore do not see what advantage could be gained from additionally imposing E2' E4' on the local models: it imposes constraints on how the values of the local models can be chosen, yet does not imply any additional properties for the global game-theoretic upper expectation ( $\overline{\mathrm{E}}_{\mathrm{V}}$ only depends on the values of the local models on $\overline{\mathscr{L}}_{b}(\mathscr{X})$ and, on this restricted domain, axioms E2'E4' are as general as E2 E4 according to Proposition 2). If one moreover finds it desirable to have complete compatibility with the local models, then one should additionally imposeE6 on the local models, irrespectively of whether he is already imposing E2'E4' at which point E2'E4'become redundant due to Proposition 1 


\section{Behaviour of $\overline{\mathbf{E}}_{\mathrm{V}}$ with respect to sequences of finitary variables}

Even though the upper expectation $\overline{\mathrm{E}}_{\mathrm{V}}$ is continuous with respect to non-decreasing sequences and with respect to non-increasing sequences of lower cuts, it is not necessarily continuous with respect to general pointwise convergence; see [20, Example 1] for an illustration 4 However, in many cases, the sequence of interest will be composed out of variables that are finitary; the individual variables then only depend on a finite number of states. Compared to general sequences, sequences of finitary variables tend to be more well-behaved and therefore allow us to establish stronger continuity results. Moreover, upper expectations of finitary variables or, more specifically, finitary gambles can often be computed rather efficiently; see [21]. If these computational methods were to be combined with the appropriate continuity properties-which tend to be stronger for sequences of finitary variables-we would also be able to compute upper expectations of a great deal of non-finitary variables.

Because of their importance in this section, we will denote the set of all bounded below variables $f \in \overline{\mathbb{V}}_{b}$ such that $f=\lim _{n \rightarrow+\infty} f_{n}$ for some sequence $\left\{f_{n}\right\}_{n \in \mathbb{N}_{0}}$ of finitary variables by $\overline{\mathbb{V}}_{b, \text { lim }}$. Our first result indicates that the variables in $\overline{\mathbb{V}}_{b \text {,lim }}$ play a crucial role in the characterisation of game-theoretic upper expectations. It states that the upper expectation $\overline{\mathrm{E}}_{\mathrm{V}}(f \mid s)$ of any variable $f \in \overline{\mathbb{V}}$, conditional on any $s \in \mathscr{X}^{*}$, is the lower envelope of the upper expectations $\overline{\mathrm{E}}_{\mathrm{V}}(g \mid s)$ of variables $g \in \overline{\mathbb{V}}_{b \text {,lim }}$ that dominate $f$ on the domain $\Gamma(s)$. Once more, this result allows us to conclude that $\overline{\mathrm{E}}_{\mathrm{V}}$ is uniquely characterised by its values on a constrained domain; that of the (bounded below) limits of finitary variables. We refer to [18, 19] for a more detailed discussion.

Proposition 28. Consider any $f \in \overline{\mathbb{V}}$ and any $s \in \mathscr{X}^{*}$. Then

$$
\overline{\mathrm{E}}_{\mathrm{V}}(f \mid s)=\inf \left\{\overline{\mathrm{E}}_{\mathrm{V}}(g \mid s): g \in \overline{\mathbb{V}}_{b, \lim } \text { and } g \geq_{s} f\right\}=\inf \left\{\overline{\mathrm{E}}_{\mathrm{V}}(g \mid s): g \in \overline{\mathbb{V}}_{b, \lim } \text { and } g \geq f\right\} \text {. }
$$

Proof. Because $\overline{\mathrm{E}}_{\mathrm{V}}$ is monotone [V4, we have that $\overline{\mathrm{E}}_{\mathrm{V}}(f \mid s) \leq \overline{\mathrm{E}}_{\mathrm{V}}(g \mid s)$ for any $g \in \overline{\mathbb{V}}_{b, \text { lim }}$ such that $f \leq_{s} g$. It therefore follows immediately that

$$
\overline{\mathrm{E}}_{\mathrm{V}}(f \mid s) \leq \inf \left\{\overline{\mathrm{E}}_{\mathrm{V}}(g \mid s): g \in \overline{\mathbb{V}}_{b, \lim } \text { and } g \geq_{s} f\right\} \leq \inf \left\{\overline{\mathrm{E}}_{\mathrm{V}}(g \mid s): g \in \overline{\mathbb{V}}_{b, \lim } \text { and } g \geq f\right\},
$$

where the last inequality follows from the fact that $g \geq f$ implies $g \geq_{s} f$ for any $g \in \overline{\mathbb{V}}$. It remains to prove that $\inf \left\{\overline{\mathrm{E}}_{\mathrm{V}}(g \mid s): g \in \overline{\mathbb{V}}_{b, \lim }\right.$ and $\left.g \geq f\right\} \leq \overline{\mathrm{E}}_{\mathrm{V}}(f \mid s)$.

Consider any $\mathscr{M}^{\prime} \in \overline{\mathbb{M}}_{\text {b }}$ such that $\lim \mathscr{M}^{\prime}$ exists within $\Gamma(s)$ and such that $\lim \mathscr{M}^{\prime} \geq_{s} f$. Let $\mathscr{M}$ be the extended real process defined by $\mathscr{M}(t):=\mathscr{M}^{\prime}(t)$ for all $t \sqsupseteq s$, and by $\mathscr{M}(t):=+\infty$ for all $t \nsupseteq s$. We show that $\mathscr{M}$ is a bounded below supermartingale such that $\lim \mathscr{M} \geq f$. The process $\mathscr{M}$ is bounded below because $\mathscr{M}^{\prime}$ is bounded below. Moreover, we have, for all $t \sqsupseteq s$, that $\overline{\mathrm{Q}}_{t}(\mathscr{M}(t \cdot))=\overline{\mathrm{Q}}_{t}\left(\mathscr{M}^{\prime}(t \cdot)\right) \leq \mathscr{M}^{\prime}(t)=\mathscr{M}(t)$ because $\mathscr{M}^{\prime}$ is a supermartingale, and, for all $t \geqq s$, we also have that $\overline{\mathrm{Q}}_{t}(\mathscr{M}(t \cdot)) \leq \mathscr{M}(t)$ because then $\mathscr{M}(t)=+\infty$. Hence, $\mathscr{M}$ is indeed a bounded below supermartingale. Furthermore, note that $\lim \mathscr{M}={ }_{s} \lim \mathscr{M}^{\prime} \geq_{s} f$ and, for any path $\omega$ not going through $s$, that $\lim \mathscr{M}(\omega)=+\infty \geq f(\omega)$, which all together implies that $\lim \mathscr{M} \geq f$.

Now, let $\left\{g_{n}\right\}_{n \in \mathbb{N}_{0}}$ be the sequence defined by $g_{n}(\omega):=\mathscr{M}\left(\omega^{n}\right)$ for all $n \in \mathbb{N}_{0}$ and all $\omega \in \Omega$. Then it is clear that $\left\{g_{n}\right\}_{n \in \mathbb{N}_{0}}$ is a sequence of $n$-measurable, and therefore finitary, extended real variables that is uniformly bounded

\footnotetext{
${ }^{4}$ The version of the game-theoretic upper expectation used in [20, Example 1] is with real-valued supermartingales instead of extended real-valued ones. However, this does not make any difference because [20, Example 1] only involves upper expectations of gambles and, on that domain, both versions of the game-theoretic upper expectation coincide; see Proposition 36 further on.
} 
below. Moreover, since $\lim \mathscr{M}$ exists everywhere, we have that $g(\omega):=\lim _{n \rightarrow+\infty} g_{n}(\omega)=\lim _{n \rightarrow+\infty} \mathscr{M}\left(\omega^{n}\right)$ exists for all $\omega \in \Omega$. Hence, $g \in \overline{\mathbb{V}}_{b, \lim }$ and because $\lim \mathscr{M} \geq f$ also $g \geq f$. It furthermore follows from Definition 3 that $\overline{\mathrm{E}}_{\mathrm{V}}(g \mid s) \leq \mathscr{M}(s)$ because $\lim \mathscr{M} \geq_{s} g$ (since, in fact, $\lim \mathscr{M}=g$ ). This implies that

$$
\inf \left\{\overline{\mathrm{E}}_{\mathrm{V}}(g \mid s): g \in \overline{\mathbb{V}}_{b, \lim } \text { and } g \geq f\right\} \leq \mathscr{M}(s)=\mathscr{M}^{\prime}(s) .
$$

Since this holds for any $\mathscr{M}^{\prime} \in \overline{\mathbb{M}}_{\mathrm{b}}$ such that $\lim \mathscr{M}^{\prime}$ exists within $\Gamma(s)$ and $\lim \mathscr{M}^{\prime} \geq_{s} f$, it follows from Proposition 20 that

$$
\inf \left\{\overline{\mathrm{E}}_{\mathrm{V}}(g \mid s): g \in \overline{\mathbb{V}}_{b, \lim } \text { and } g \geq f\right\} \leq \overline{\mathrm{E}}_{\mathrm{V}}(f \mid s) \text {. }
$$

The result above is expressed in terms of (bounded below) limits of finitary variables, but we could just as well have replaced them by (bounded below) limits of $n$-measurable gambles because, as we intend to prove next, each $f \in \overline{\mathbb{V}}_{b \text {,lim }}$ is a pointwise limit of a sequence of $n$-measurable gambles. As a first step to establish this result, we aim to transition from any sequence $\left\{g_{n}\right\}_{n \in \mathbb{N}_{0}}$ of finitary variables to a sequence $\left\{g_{n}^{\xi}\right\}_{n \in \mathbb{N}_{0}}$ of $n$-measurable variables, without changing the essential characteristics of the sequence. To this end, let $\left\{g_{n}^{\xi}\right\}_{n \in \mathbb{N}_{0}}$ and $\xi: \mathbb{N} \rightarrow \mathbb{N}_{0}$ be defined by the following recursive expressions, where $g_{0}^{\xi}:=c \in \overline{\mathbb{R}}$ is a freely chosen extended real number and $\xi(1):=0$ :

$$
g_{n}^{\xi}:=\left\{\begin{array}{ll}
g_{\xi(n)} & \text { if } g_{\xi(n)} \text { is } n \text {-measurable; } \\
g_{n-1}^{\xi} & \text { otherwise, }
\end{array} \quad \text { and } \quad \xi(n+1):= \begin{cases}\xi(n)+1 & \text { if } g_{\xi(n)} \text { is } n \text {-measurable } \\
\xi(n) & \text { otherwise }\end{cases}\right.
$$

for all $n \in \mathbb{N}$. Note that $\left\{g_{n}\right\}_{n \in \mathbb{N}_{0}}$ is a subsequence of $\left\{g_{n}^{\xi}\right\}_{n \in \mathbb{N}_{0}}$ and that the additional terms in $\left\{g_{n}^{\xi}\right\}_{n \in \mathbb{N}_{0}}$ do not impact the limit behaviour, nor, for a suitable choice of $c$, the (possibly) monotone character of the original sequence.

Lemma 29. For any sequence $\left\{g_{n}\right\}_{n \in \mathbb{N}_{0}}$ of finitary variables, the sequence $\left\{g_{n}^{\xi}\right\}_{n \in \mathbb{N}_{0}}$ is a sequence of $n$-measurable variables.

Proof. We prove this by induction. $g_{0}^{\xi}=c$ is clearly 0 -measurable. To prove the induction step, suppose that $g_{k-1}^{\xi}$ is $(k-1)$-measurable for some $k \in \mathbb{N}$. Then either we have that $g_{\xi(k)}$ is $k$-measurable, which directly implies that $g_{k}^{\xi}=g_{\xi(k)}$ is $k$-measurable. Otherwise, $g_{k}^{\xi}$ is equal to $g_{k-1}^{\xi}$ implying that $g_{k}^{\xi}$ is $(k-1)$-measurable and therefore automatically $k$-measurable. This concludes the induction step and hence, $\left\{g_{n}^{\xi}\right\}_{n \in \mathbb{N}_{0}}$ is a sequence of $n$-measurable variables.

We now establish our earlier claim that the variables in $\overline{\mathbb{V}}_{b \text {,lim }}$ are essentially limits of $n$-measurable gambles and therefore, that Proposition 28 turns out to be stronger than it first appears.

Proposition 30. For any $f \in \overline{\mathbb{V}}_{b, \mathrm{lim}}, f$ is the pointwise limit of a sequence $\left\{f_{n}\right\}_{n \in \mathbb{N}_{0}}$ of $n$-measurable gambles. Furthermore, we can guarantee that $B \leq f_{n} \leq \sup f$ for all $n \in \mathbb{N}_{0}$, where $B$ is any real if $\inf f=+\infty$ and $B=\inf f$ otherwise.

Proof. Fix any $f \in \overline{\mathbb{V}}_{b, \text { lim }}$. Then, according to the definition of $\overline{\mathbb{V}}_{b, \lim }, f$ is the pointwise limit of a sequence $\left\{g_{n}\right\}_{n \in \mathbb{N}_{0}}$ of finitary variables. Let $\left\{g_{n}^{\xi}\right\}_{n \in \mathbb{N}_{0}}$ be the sequence defined by the recursive expression [11], with $c=0$, 
which by Lemma29 is a sequence of $n$-measurable variables. As explained in the text that preceeds Lemma29, the sequences $\left\{g_{n}\right\}_{n \in \mathbb{N}_{0}}$ and $\left\{g_{n}^{\xi}\right\}_{n \in \mathbb{N}_{0}}$ have the same limit behaviour, so $\left\{g_{n}^{\xi}\right\}_{n \in \mathbb{N}_{0}}$ converges pointwise to $f$. Let $B$ be any real if $\inf f=+\infty$ and let $B:=\inf f$ if $\inf f \in \mathbb{R}$ [the case where $\inf f=-\infty$ is impossible because $f$ is bounded below]. Let $\left\{f_{n}\right\}_{n \in \mathbb{N}_{0}}$ be the sequence defined by bounding each $g_{n}^{\xi}$ above by $\min \{n$, sup $f\}$ and below by $B$; so $f_{n}(\omega):=\max \left\{\min \left\{g_{n}^{\xi}(\omega), n, \sup f\right\}, B\right\}$ for all $\omega \in \Omega$ and all $n \in \mathbb{N}_{0}$. Then it is clear that $\left\{f_{n}\right\}_{n \in \mathbb{N}_{0}}$ is a sequence of $n$-measurable gambles because $\left\{g_{n}^{\xi}\right\}_{n \in \mathbb{N}_{0}}$ is a sequence of $n$-measurable (possibly extended real) variables. It also converges pointwise to $f$ because

$$
\begin{aligned}
f(\omega)=\max \{\min \{f(\omega), \sup f\}, B\} & =\max \left\{\min \left\{\lim _{n \rightarrow+\infty} g_{n}^{\xi}(\omega), \lim _{n \rightarrow+\infty} n, \sup f\right\}, B\right\} \\
& =\lim _{n \rightarrow+\infty} \max \left\{\min \left\{g_{n}^{\xi}(\omega), n, \sup f\right\}, B\right\}=\lim _{n \rightarrow+\infty} f_{n}(\omega),
\end{aligned}
$$

for all $\omega \in \Omega$, where the first equality follows from the fact that $B \leq \inf f \leq f$. Moreover, for all $n \in \mathbb{N}_{0}$, we clearly have that $B \leq f_{n}$, and also $f_{n} \leq \sup f$ because $\min \left\{g_{n}^{\xi}(\omega), n, \sup f\right\} \leq \sup f$ for all $\omega \in \Omega$ and $B \leq \inf f \leq \sup f$. Hence, $\left\{f_{n}\right\}_{n \in \mathbb{N}_{0}}$ satisfies all of the conditions in the proposition.

We now present two main results. The first one states that $\overline{\mathrm{E}}_{\mathrm{V}}$ is continuous with respect to non-increasing sequences of finitary bounded above variables. The second one says that, for any $f \in \overline{\mathbb{V}}_{b \text {,lim }}$, there is always a sequence of $n$-measurable gambles—and therefore also a sequence of finitary gambles—that converges pointwise to $f$ and for which $\overline{\mathrm{E}}_{\mathrm{V}}$ is continuous.

Theorem 31. For any $s \in \mathscr{X}^{*}$ and any non-increasing sequence $\left\{f_{n}\right\}_{n \in \mathbb{N}_{0}}$ of finitary, bounded above variables that converges pointwise to a variable $f \in \overline{\mathbb{V}}$, we have that $\lim _{n \rightarrow+\infty} \overline{\mathrm{E}}_{\mathrm{V}}\left(f_{n} \mid s\right)=\overline{\mathrm{E}}_{\mathrm{V}}(f \mid s)$.

Theorem 32. For any $s \in \mathscr{X}^{*}$ and any $f \in \overline{\mathbb{V}}_{b, \lim }$, there is a sequence $\left\{f_{n}\right\}_{n \in \mathbb{N}_{0}}$ of $n$-measurable gambles that is uniformly bounded below and that converges pointwise to $f$ such that $\lim _{n \rightarrow+\infty} \overline{\mathrm{E}}_{\mathrm{V}}\left(f_{n} \mid s\right)=\overline{\mathrm{E}}_{\mathrm{V}}(f \mid s)$.

Both of the results above have already led to valuable theoretical insights in the literature: Theorem 31 was crucial to obtain an equivalence result about hitting times and probabilities in imprecise Markov chains [9]. Theorem 32 on the other hand, further establishes the importance of finitary variables and their limits when it comes to characterising $\overline{\mathrm{E}}_{\mathrm{V}}$ [18, 19]. In fact, Theorem 32 was a key result in obtaining our alternative characterisation presented in [18] (or, more recently, the characterisation presented in [19]). For this reason, a version of Theorem 32 was also already included in an online report [17] that serves as a technical reference for [18].

The remainder of this section is devoted to the proofs of Theorem 31 and 32 We start with two technical lemmas that are key in proving them both.

Lemma 33. Consider any global variable $h \in \overline{\mathbb{V}}$ taking values in $\mathbb{N}_{0}$. If $h(\omega)=h(\tilde{\omega})$ for every $\omega \in \Omega$ and $\tilde{\omega} \in$ $\Gamma\left(\omega^{h(\omega)}\right)$, then $h$ is bounded below and above — and therefore a gamble—and $h$ is $(\sup h)$-measurable, with $\sup h:=$ $\sup _{\omega \in \Omega} h(\omega) \in \mathbb{N}_{0}$.

Proof. $h$ is clearly bounded below because it takes value in $\mathbb{N}_{0}$. Assume ex absurdo that $h$ is not bounded above; so $\sup h=+\infty$. Then we have that

$$
\sup _{\omega \in \Omega} h(\omega)=\sup _{x_{1} \in \mathscr{X}} \sup _{\omega \in \Gamma\left(x_{1}\right)} h(\omega)=+\infty .
$$


Since $\mathscr{X}$ is finite, there is clearly some $x_{1}^{*} \in \mathscr{X}$ for which $\sup _{\omega \in \Gamma\left(x_{1}^{*}\right)} h(\omega)=+\infty$. Similarly, we also find that

$$
\sup _{\omega \in \Gamma\left(x_{1}^{*}\right)} h(\omega)=\sup _{x_{2} \in \mathscr{X}} \sup _{\omega \in \Gamma\left(x_{1}^{*} x_{2}\right)} h(\omega)=+\infty
$$

Since $\mathscr{X}$ is finite, there is again some $x_{2}^{*} \in \mathscr{X}$ for which $\sup _{\omega \in \Gamma\left(x_{1}^{*} x_{2}^{*}\right)} h(\omega)=+\infty$. We can continue in this way and construct a path $\omega=x_{1}^{*} x_{2}^{*} \ldots x_{n}^{*} \ldots$ for which

$$
\sup _{\omega^{\prime} \in \Gamma\left(\omega^{n}\right)} h\left(\omega^{\prime}\right)=+\infty \text { for all } n \in \mathbb{N}_{0}
$$

However, $h$ takes values in $\mathbb{N}_{0}$, so $h(\omega) \in \mathbb{N}_{0}$. This implies, together with the assumption that $h\left(\omega^{\prime}\right)=h(\omega)$ for every $\omega^{\prime} \in \Gamma\left(\omega^{h(\omega)}\right)$, that

$$
\sup \left\{h\left(\omega^{\prime}\right): \omega^{\prime} \in \Gamma\left(\omega^{h(\omega)}\right)\right\}=\sup \left\{h(\omega): \omega^{\prime} \in \Gamma\left(\omega^{h(\omega)}\right)\right\}=h(\omega) \in \mathbb{N}_{0}
$$

This is in contradiction with Equation [12] [for $n=h(\omega)$ ], so $h$ is bounded above, which together with the fact that $h$ is bounded below, implies that $h$ is a gamble. The fact that $h$ is bounded above and that it takes values in $\mathbb{N}_{0}$, also clearly implies that sup $h \in \mathbb{N}_{0}$. To see that $h$ is ( $\left.\sup h\right)$-measurable, consider any $\omega \in \Omega$ and any $\tilde{\omega} \in \Gamma\left(\omega^{\sup h}\right)$. Then $\tilde{\omega} \in \Gamma\left(\omega^{h(\omega)}\right)$ because $h(\omega) \leq \sup h$ and therefore, by assumption, we have that $h(\omega)=h(\tilde{\omega})$.

For the following technical lemma, we will associate with any sequence $\left\{f_{n}\right\}_{n \in \mathbb{N}_{0}}$ of $n$-measurable gambles and any global variable $h \in \overline{\mathbb{V}}$ taking values in $\mathbb{N}_{0}$, the global variable $f_{h} \in \overline{\mathbb{V}}$ defined by $f_{h}(\omega):=f_{h(\omega)}(\omega)$. We will also need the notion of a cut $U \subset \mathscr{X}^{*}$ : a collection of pairwise incomparable situations. We call a cut $U$ complete if for all $\omega \in \Omega$ there is some $u \in U$ such that $\omega \in \Gamma(u)$. Otherwise, we call $U$ partial. We will also use the simpler notation $s$ to denote the cut $\{s\}$ that consists of the single situation $s \in \mathscr{X}^{*}$. For any situation $s$ and any cut $U$, we write $s \sqsubset U$ if $s \sqsubset u$ for all $u \in U$. So, if $s \neq \square$ and $s \sqsubset U$, then $U$ must be partial. Conversely, we write $U \sqsubset s$ if there is a $u \in U$ such that $u \sqsubset s$. In a similar way, we extend the relations $\sqsubseteq, \sqsupset$ and $\sqsupseteq$. Analogously to what we did before for situations, we say that a path $\omega \in \Omega$ goes through a cut $U$ when there is some $n \in \mathbb{N}_{0}$ such that $\omega^{n} \in U$.

Lemma 34. Consider any $s \in \mathscr{X}^{*}$ and any sequence $\left\{f_{n}\right\}_{n \in \mathbb{N}_{0}}$ of $n$-measurable gambles that converges pointwise to a variable $f \in \overline{\mathbb{V}}$ that is bounded above. Then, for any $m \in \mathbb{N}_{0}$ and any $\alpha \in \mathbb{R}$ such that $\overline{\mathrm{E}}_{\mathrm{V}}(f \mid s)<\alpha$, there is a gamble $h: \Omega \rightarrow \mathbb{N}_{0}$ that is (sup $h$ )-measurable such that $m \leq h$ and $\overline{\mathrm{E}}_{\mathrm{V}}\left(f_{h} \mid s\right) \leq \alpha$.

Proof. Fix any $m \in \mathbb{N}_{0}$, any $\alpha \in \mathbb{R}$ such that $\overline{\mathrm{E}}_{\mathrm{V}}(f \mid s)<\alpha$ and any $\epsilon \in \mathbb{R}_{>0}$. According to the definition of $\overline{\mathrm{E}}_{\mathrm{V}}(f \mid s)$, there is a supermartingale $\mathscr{M} \in \overline{\mathbb{M}}_{\mathrm{b}}$ such that $\mathscr{M}(s) \leq \alpha$ and $\liminf \mathscr{M} \geq_{s} f$. We start by showing that, for any $\omega \in \Gamma(s)$ and any $n^{*} \in \mathbb{N}_{0}$, there is a natural number $n \geq n^{*}$ such that $\mathscr{M}\left(\omega^{n}\right)+\epsilon \geq f_{n}(\omega)$.

So consider any $\omega \in \Gamma(s)$. First note that $\liminf \mathscr{M}(\omega)+\epsilon>f(\omega)$ because $\liminf \mathscr{M} \geq_{s} f$, $\liminf \mathscr{M}(\omega)>$ $-\infty$ [ $\mathscr{M}$ is bounded below] and $f(\omega)<+\infty$ [ $f$ is bounded above]. This implies that there is a real $\beta$ such that $\liminf \mathscr{M}(\omega)+\epsilon>\beta>f(\omega)$. Then, since $\left\{f_{n}(\omega)\right\}_{n \in \mathbb{N}_{0}}$ converges to $f(\omega)$ and $\beta$ is a real such that $\beta>f(\omega)$, there is an index $N(\omega) \in \mathbb{N}_{0}$ such that $\beta>f_{n}(\omega)$ for all $n \geq N(\omega)$. Furthermore, by the definition of the limit inferior and the fact that $\beta$ is a real such that $\liminf \mathscr{M}(\omega)+\epsilon>\beta$, there is a second index $M(\omega) \in \mathbb{N}_{0}$ such that $\mathscr{M}\left(\omega^{n}\right)+\epsilon>\beta$ for all $n \geq M(\omega)$. Combined with the previous, we obtain that $\mathscr{M}\left(\omega^{n}\right)+\epsilon>\beta>f_{n}(\omega)$ for all $n \geq \max \{N(\omega), M(\omega)\}$. Since this holds for all $n \geq \max \{N(\omega), M(\omega)\}$, there is for any $n^{*} \in \mathbb{N}_{0}$ also an $n \geq n^{*}$ such that $\mathscr{M}\left(\omega^{n}\right)+\epsilon \geq f_{n}(\omega)$. 
Let $\ell$ be the length of the string $s$ and consider the variable $h \in \overline{\mathbb{V}}$ defined by

$$
h(\omega):=\left\{\begin{array}{ll}
\inf \left\{n \geq \max \{\ell, m\}: \mathscr{M}\left(\omega^{n}\right)+\epsilon \geq f_{n}(\omega)\right\} & \text { if } \omega \in \Gamma(s) ; \\
\max \{\ell, m\} & \text { otherwise, }
\end{array} \text { for all } \omega \in \Omega\right.
$$

It clearly follows from the argument above that $h$ takes values in $\mathbb{N}_{0}$. We will now moreover show that $h(\omega)=h(\tilde{\omega})$ for any $\omega \in \Omega$ and any $\tilde{\omega} \in \Gamma\left(\omega^{h(\omega)}\right)$, implying that $h$ satisfies the conditions in Lemma33.

Consider any $\omega \in \Omega$ and any $\tilde{\omega} \in \Gamma\left(\omega^{h(\omega)}\right)$. We distinguish two cases: $\omega \in \Gamma(s)$ and $\omega \notin \Gamma(s)$. If $\omega \in \Gamma(s)$, then it follows from the definition of $h$ that $\mathscr{M}\left(\omega^{h(\omega)}\right)+\epsilon \geq f_{h(\omega)}(\omega)$. Since $\omega^{h(\omega)}=\tilde{\omega}^{h(\omega)}$ [because $\tilde{\omega} \in \Gamma\left(\omega^{h(\omega)}\right)$ ] and since $f_{h(\omega)}$ is $h(\omega)$-measurable, this implies that $\mathscr{M}\left(\tilde{\omega}^{h(\omega)}\right)+\epsilon \geq f_{h(\omega)}(\tilde{\omega})$. Then, according to the definition of $h$ and since $\tilde{\omega} \in \Gamma(s)$ [because $h(\omega) \geq \ell$ and $\omega \in \Gamma(s)$, and therefore $\tilde{\omega} \in \Gamma\left(\omega^{h(\omega)}\right) \subseteq \Gamma(s)$ ], we have that $h(\tilde{\omega}) \leq h(\omega)$. On the other hand, since $\tilde{\omega} \in \Gamma(s)$ and $\omega \in \Gamma\left(\tilde{\omega}^{h(\tilde{\omega})}\right)$ [because $\omega^{h(\omega)}=\tilde{\omega}^{h(\omega)}$ and $h(\tilde{\omega}) \leq h(\omega)$ ], we can infer, in exactly the same way as before, that $h(\omega) \leq h(\tilde{\omega})$. So we conclude that $h(\omega)=h(\tilde{\omega})$ in case that $\omega \in \Gamma(s)$. If $\omega \notin \Gamma(s)$, then $\tilde{\omega} \notin \Gamma(s)$ because $h(\omega) \geq \ell$ and therefore $\Gamma\left(\omega^{h(\omega)}\right) \cap \Gamma(s)=\emptyset$. Then it follows immediately from the definition of $h$ that $h(\omega)=h(\tilde{\omega})$. Hence, $h$ satisfies the conditions in Lemma 33, so we have that $\sup h \in \mathbb{N}_{0}$ and that $h$ is a ( $\sup h$ )-measurable gamble. Furthermore, we trivially have that $h \geq \ell$ and $h \geq m$.

Let $U:=\left\{t \in \mathscr{X}^{*}:(\exists \omega \in \Omega) t=\omega^{h(\omega)}\right\}$. Note that any two (different) situations $t$ and $u$ in $U$ are incomparable. Indeed, assume ex absurdo that this is not the case. Then there are at least two different situations $t, u \in U$ such that $t \sqsubseteq u$. Let $\omega$ and $\tilde{\omega}$ be two paths such that $t=\omega^{h(\omega)}$ and $u=\tilde{\omega}^{h(\tilde{\omega})}$. Since $t \sqsubseteq u$, we have that $\tilde{\omega} \in \Gamma(t)=\Gamma\left(\omega^{h(\omega)}\right)$, which due to our previous considerations implies that $h(\tilde{\omega})=h(\omega)$. Hence, taking into account that $\tilde{\omega} \in \Gamma\left(\omega^{h(\omega)}\right)$, we infer that $\omega^{h(\omega)}=\tilde{\omega}^{h(\tilde{\omega})}$ and therefore that $t=u$. This is in contradiction with our assumption that $t$ and $u$ are different, so we conclude that all situations in $U$ are pairwise incomparable and therefore, that $U$ is a cut. $U$ is also a complete cut. To see why, observe that since $h$ takes values in $\mathbb{N}_{0}$, it follows that, for any $\omega \in \Omega, \omega^{h(\omega)}$ is a situation, which by definition is an element of $U$; it is moreover clear that for this situation $\omega^{h(\omega)}$, we have that $\omega \in \Gamma\left(\omega^{h(\omega)}\right)$. Hence, $U$ is a complete cut, because for all $\omega \in \Omega$, there is a situation $u \in U$ such that $\omega \in \Gamma(u)$.

For any situation $t \sqsupseteq U$, let us write $u(t)$ to denote the unique situation in $U$ such that $u(t) \sqsubseteq t$. That $u(t)$ is unique follows from the fact that the situations in $U$ are incomparable. Indeed, if there would be a second $u^{\prime}(t) \in U$ such that $u^{\prime}(t) \sqsubseteq t$, this would imply that either $u(t) \sqsubseteq u^{\prime}(t)$ or $u^{\prime}(t) \sqsubseteq u(t)$, which is impossible since $u(t)$ and $u^{\prime}(t)$ are incomparable. Now let $\mathscr{M}_{U}$ be the extended real process defined by

$$
\mathscr{M}_{U}(t):=\left\{\begin{array}{ll}
\mathscr{M}(u(t)) & \text { if } U \sqsubset t ; \\
\mathscr{M}(t) & \text { if } U \not \subset t,
\end{array} \text { for all } t \in \mathscr{X}^{*} .\right.
$$

The process $\mathscr{M}_{U}$ is bounded below because $\mathscr{M}$ is bounded below. To see that $\mathscr{M}_{U}$ is a supermartingale, note that

$$
\mathscr{M}_{U}(t \cdot):=\left\{\begin{array}{ll}
\mathscr{M}(u(t)) & \text { if } U \sqsubseteq t ; \\
\mathscr{M}(t \cdot) & \text { if } U \nsubseteq t,
\end{array} \text { for all } t \in \mathscr{X}^{*} .\right.
$$

So for any situation $t \sqsupseteq U$, we have that $\mathscr{M}_{U}(t)=\mathscr{M}(u(t))$ and that $\mathscr{M}_{U}(t \cdot)=\mathscr{M}(u(t))$, which implies that $\overline{\mathrm{Q}}_{t}\left(\mathscr{M}_{U}(t \cdot)\right)=\mathscr{M}_{U}(t)$ because of E7 On the other hand, for any situation $t \nsupseteq U$, we also have that $\overline{\mathrm{Q}}_{t}\left(\mathscr{M}_{U}(t \cdot)\right) \leq$ 
$\mathscr{M}_{U}(t)$ because $\overline{\mathrm{Q}}_{t}(\mathscr{M}(t \cdot)) \leq \mathscr{M}(t)\left[\mathscr{M}\right.$ is a supermartingale]. As a consequence, $\mathscr{M}_{U}$ is a bounded below supermartingale.

For any $\omega \in \Omega$, we now let $u(\omega)$ be the unique situation in $U$ such that $\omega \in \Gamma(u(\omega))$. Then clearly $u(\omega)=\omega^{h(\omega)}$. Moreover, for an $m \in \mathbb{N}_{0}$ large enough such that $U \sqsubset \omega^{m}$, we also have that $\mathscr{M}_{U}\left(\omega^{m}\right)=\mathscr{M}\left(u\left(\omega^{m}\right)\right)=\mathscr{M}(u(\omega))$. Hence,

$$
\lim _{m \rightarrow+\infty} \mathscr{M}_{U}\left(\omega^{m}\right)=\lim _{m \rightarrow+\infty} \mathscr{M}(u(\omega))=\mathscr{M}(u(\omega))=\mathscr{M}\left(\omega^{h(\omega)}\right) \text { for all } \omega \in \Omega
$$

Therefore, by definition of $h$, we have that

$$
\lim _{m \rightarrow+\infty} \mathscr{M}_{U}\left(\omega^{m}\right)+\epsilon=\mathscr{M}\left(\omega^{h(\omega)}\right)+\epsilon \geq f_{h(\omega)}(\omega)=: f_{h}(\omega) \text { for all } \omega \in \Gamma(s) .
$$

Then by Definition 3 and taking into account that $\mathscr{M}_{U} \in \overline{\mathbb{M}}_{\mathrm{b}}$ and therefore $\left[\mathscr{M}_{U}+\epsilon\right] \in \overline{\mathbb{M}}_{\mathrm{b}}$ [because the local models $\overline{\mathrm{Q}}_{s}$ satisfyE8], it follows that $\overline{\mathrm{E}}_{\mathrm{V}}\left(f_{h} \mid s\right) \leq \mathscr{M}_{U}(s)+\epsilon$. Moreover, $\mathscr{M}_{U}(s)=\mathscr{M}(s)$ because $h \geq \ell$ and therefore $U \not \subset s$, so we also have that $\overline{\mathrm{E}}_{\mathrm{V}}\left(f_{h} \mid s\right) \leq \mathscr{M}(s)+\epsilon \leq \alpha+\epsilon$. Since this inequality holds for any $\epsilon \in \mathbb{R}_{>0}$, we infer that $\overline{\mathrm{E}}_{\mathrm{V}}\left(f_{h} \mid s\right) \leq \alpha$, which together with the fact that $h$ is a ( $\sup h$ )-measurable gamble such that $m \leq h$, establishes the lemma.

The idea underlying the proof of Theorem 31 originates in a result by some of us [3, Theorem 3], however, once more, real supermartingales were adopted there. Moreover, our result here considers sequences of (extended real) finitary variables that are bounded above, instead of sequences of $n$-measurable gambles.

Proof of Theorem 31. Fix any $s \in \mathscr{X}^{*}$ and any non-increasing sequence $\left\{f_{n}\right\}_{n \in \mathbb{N}_{0}}$ of finitary, bounded above variables that converges pointwise to a variable $f \in \overline{\mathbb{V}}$. Note that $f$ is then also bounded above. Because $f_{n} \geq f_{n+1} \geq f$ for all $n \in \mathbb{N}_{0}$ and $\overline{\mathrm{E}}_{\mathrm{V}}$ is monotone [V4], the $\operatorname{limit}_{\lim } \lim _{n \rightarrow+\infty} \overline{\mathrm{E}}_{\mathrm{V}}\left(f_{n} \mid s\right)$ exists and we have that $\lim _{n \rightarrow+\infty} \overline{\mathrm{E}}_{\mathrm{V}}\left(f_{n} \mid s\right) \geq$ $\overline{\mathrm{E}}_{\mathrm{V}}(f \mid s)$. So we are left to show that $\lim _{n \rightarrow+\infty} \overline{\mathrm{E}}_{\mathrm{V}}\left(f_{n} \mid s\right) \leq \overline{\mathrm{E}}_{\mathrm{V}}(f \mid s)$.

Consider the sequence $\left\{f_{n}^{\vee-n}\right\}_{n \in \mathbb{N}_{0}}$ and note that it suffices to show that $\lim _{n \rightarrow+\infty} \overline{\mathrm{E}}_{\mathrm{V}}\left(f_{n}^{\vee-n} \mid s\right) \leq \overline{\mathrm{E}}_{\mathrm{V}}(f \mid s)$, where the limit $\lim _{n \rightarrow+\infty} \overline{\mathrm{E}}_{\mathrm{V}}\left(f_{n}^{\vee-n} \mid s\right)$ exists because $\left\{f_{n}^{\vee-n}\right\}_{n \in \mathbb{N}_{0}}$ is clearly non-increasing [since $\left\{f_{n}\right\}_{n \in \mathbb{N}_{0}}$ is non-increasing] and $\overline{\mathrm{E}}_{\mathrm{V}}$ is monotone [V4]. Indeed, it will then follow that $\lim _{n \rightarrow+\infty} \overline{\mathrm{E}}_{\mathrm{V}}\left(f_{n} \mid s\right) \leq \overline{\mathrm{E}}_{\mathrm{V}}(f \mid s)$ because $f_{n} \leq f_{n}^{\vee-n}$ for all $n \in \mathbb{N}_{0}$ and therefore, by 44 that $\lim _{n \rightarrow+\infty} \overline{\mathrm{E}}_{\mathrm{V}}\left(f_{n} \mid s\right) \leq \lim _{n \rightarrow+\infty} \overline{\mathrm{E}}_{\mathrm{V}}\left(f_{n}^{\vee-n} \mid s\right) \leq \overline{\mathrm{E}}_{\mathrm{V}}(f \mid s)$.

Since $\left\{f_{n}\right\}_{n \in \mathbb{N}_{0}}$ is a sequence of finitary variables that converges non-increasingly to $f$, the same holds for the sequence $\left\{f_{n}^{\vee-n}\right\}_{n \in \mathbb{N}_{0}}$. In fact, $\left\{f_{n}^{\vee-n}\right\}_{n \in \mathbb{N}_{0}}$ is even a sequence of finitary gambles because each $f_{n}$ is bounded above. Now let $g_{n}:=f_{n}^{\vee-n}$ for all $n \in \mathbb{N}_{0}$ and consider the sequence $\left\{g_{n}^{\xi}\right\}_{n \in \mathbb{N}_{0}}$ defined by the recursive expression [11, with $c \in \mathbb{R}$ such that $c \geq g_{0}$. Due to Lemma 29 , $\left\{g_{n}^{\xi}\right\}_{n \in \mathbb{N}_{0}}$ is a sequence of $n$-measurable variables. Since $\left\{g_{n}\right\}_{n \in \mathbb{N}_{0}}=\left\{f_{n}^{\vee-n}\right\}_{n \in \mathbb{N}_{0}}$ is a sequence of finitary gambles that converges non-increasingly to $f$, it follows from Equation (11) and our choice of $c$ that $\left\{g_{n}^{\xi}\right\}_{n \in \mathbb{N}_{0}}$ is a sequence of $n$-measurable gambles that converges nonincreasingly to $f$. Indeed, it can easily be checked that the transition from $\left\{g_{n}\right\}_{n \in \mathbb{N}_{0}}$ to $\left\{g_{n}^{\xi}\right\}_{n \in \mathbb{N}_{0}}$ preserves limit behaviour, the non-increasing character and the fact that the individual elements of the sequence are gambles. In the same way, we can also see that

$$
\lim _{n \rightarrow+\infty} \overline{\mathrm{E}}_{\mathrm{V}}\left(f_{n}^{\vee-n} \mid s\right)=\lim _{n \rightarrow+\infty} \overline{\mathrm{E}}_{\mathrm{V}}\left(g_{n} \mid s\right)=\lim _{n \rightarrow+\infty} \overline{\mathrm{E}}_{\mathrm{V}}\left(g_{n}^{\xi} \mid s\right)
$$


Consider any real $\alpha>\overline{\mathrm{E}}_{\mathrm{V}}(f \mid s)$, which is guaranteed to exist because $f$ is bounded above and therefore, by $\nabla 1$, $\overline{\mathrm{E}}_{\mathrm{V}}(f \mid s)<+\infty$. Then since $\left\{g_{n}^{\xi}\right\}_{n \in \mathbb{N}_{0}}$ is a sequence of $n$-measurable gambles that converges non-increasingly to $f$, Lemma 34 implies that there is a gamble $h: \Omega \rightarrow \mathbb{N}_{0}$ that is ( $\sup h$ )-measurable and is such that $\overline{\mathrm{E}}_{\mathrm{V}}\left(g_{h}^{\xi} \mid s\right) \leq \alpha$ [we simply let $m=0$ in the lemma]. Since $\left\{g_{n}^{\xi}\right\}_{n \in \mathbb{N}_{0}}$ is non-increasing and $\overline{\mathrm{E}}_{\mathrm{V}}$ is monotone [V4], we have that $\overline{\mathrm{E}}_{\mathrm{V}}\left(g_{h}^{\xi} \mid s\right) \geq \overline{\mathrm{E}}_{\mathrm{V}}\left(g_{\text {sup } h}^{\xi} \mid s\right) \geq \lim _{n \rightarrow+\infty} \overline{\mathrm{E}}_{\mathrm{V}}\left(g_{n}^{\xi} \mid s\right)$, so we infer that $\lim _{n \rightarrow+\infty} \overline{\mathrm{E}}_{\mathrm{V}}\left(g_{n}^{\xi} \mid s\right) \leq \alpha$. Recalling Equation (13), it follows that $\lim _{n \rightarrow+\infty} \overline{\mathrm{E}}_{\mathrm{V}}\left(f_{n}^{\vee-n} \mid s\right) \leq \alpha$. Since this holds for any real $\alpha>\overline{\mathrm{E}}_{\mathrm{V}}(f \mid s)$, we conclude that $\lim _{n \rightarrow+\infty} \overline{\mathrm{E}}_{\mathrm{V}}\left(f_{n}^{\vee-n} \mid s\right) \leq$ $\overline{\mathrm{E}}_{\mathrm{V}}(f \mid s)$ as desired.

For any countable net $\left\{c_{(m, n)}\right\}_{m, n \in \mathbb{N}_{0}}$ in $\overline{\mathbb{R}}$, we say that $c:=\lim _{(m, n) \rightarrow+\infty} c_{(m, n)}$ is the Moore-Smith limit of $\left\{c_{(m, n)}\right\}_{m, n \in \mathbb{N}_{0}}$ if, for each neighbourhood $A$ of $c$, there is a couple $\left(m^{*}, n^{*}\right) \in \mathbb{N}_{0}^{2}$ such that $c_{(m, n)} \in A$ for all $m \geq m^{*}$ and all $n \geq n^{*}$. Then, for any countable net $\left\{f_{(m, n)}\right\}_{m, n \in \mathbb{N}_{0}}$ in $\overline{\mathbb{V}}$ such that $\lim _{(m, n) \rightarrow+\infty} f_{(m, n)}(\omega)$ exists for all $\omega \in \Omega$, we write $\lim _{(m, n) \rightarrow+\infty} f_{(m, n)}$ to denote the variable in $\overline{\mathbb{V}}$ defined by $\lim _{(m, n) \rightarrow+\infty} f_{(m, n)}(\omega)$ for all $\omega \in \Omega$.

Lemma 35. Consider any sequence $\left\{f_{n}\right\}_{n \in \mathbb{N}_{0}}$ in $\mathbb{V}$ that converges pointwise to some variable $f \in \overline{\mathbb{V}}_{b}$. Then we have that $\lim _{(m, n) \rightarrow+\infty} f_{n}^{\wedge m}=f$.

Proof. Consider any $\omega \in \Omega$. First consider the case that $f(\omega) \in \mathbb{R}$ and fix any $\epsilon \in \mathbb{R}_{>0}$. Then there is an $n^{*} \in \mathbb{N}_{0}$ such that $\left|f_{n}(\omega)-f(\omega)\right| \leq \epsilon$ for all $n \geq n^{*}$. Consider any $m^{*} \geq f(\omega)+\epsilon$. Then for all $n \geq n^{*}$ and all $m \geq m^{*}$, we have that $f_{n}(\omega) \leq f(\omega)+\epsilon \leq m$, so $f_{n}^{\wedge m}(\omega)=f_{n}(\omega)$ and therefore $\left|f_{n}^{\wedge m}(\omega)-f(\omega)\right|=\left|f_{n}(\omega)-f(\omega)\right| \leq \epsilon$. So we have that $\lim _{(m, n) \rightarrow+\infty} f_{n}^{\wedge m}(\omega)=f(\omega)$. If $f(\omega)=+\infty$, fix any $\alpha>0$. Then there is an $n^{*} \in \mathbb{N}_{0}$ such that $f_{n}(\omega) \geq \alpha$ for all $n \geq n^{*}$. If we now take $m^{*} \geq \alpha$, then clearly also $f_{n}^{\wedge m}(\omega) \geq \alpha$ for all $n \geq n^{*}$ and all $m \geq m^{*}$. Hence, we have that $\lim _{(m, n) \rightarrow+\infty} f_{n}^{\wedge m}(\omega)=f(\omega)$ which, together with our earlier considerations, allows us to conclude that $\lim _{(m, n) \rightarrow+\infty} f_{n}^{\wedge m}=f$.

Proof of Theorem 32. Fix any $s \in \mathscr{X}^{*}$ and any $f \in \overline{\mathbb{V}}_{b, \text { lim. }}$. According to Proposition 30, there is a sequence $\left\{f_{n}\right\}_{n \in \mathbb{N}_{0}}$ of $n$-measurable gambles that converges pointwise to $f$ and such that $B \leq f_{n} \leq \sup f$ for all $n \in \mathbb{N}_{0}$, where $B$ is any real if $\inf f=+\infty$ and $B:=\inf f \operatorname{if} \inf f \in \mathbb{R}[\inf f=-\infty$ is impossible because $f$ is bounded below]. Fix any $\ell \in \mathbb{N}$ and note that the sequence $\left\{f_{n}^{\wedge \ell}\right\}_{n \in \mathbb{N}_{0}}$ is a sequence of $n$-measurable gambles that converges pointwise to $f^{\wedge \ell}$ because $\left\{f_{n}\right\}_{n \in \mathbb{N}_{0}}$ is a sequence of $n$-measurable gambles that converges pointwise to $f$. Moreover, $f^{\wedge \ell}$ is bounded above by $\ell$, so Lemma 34 guarantees that, for any $m \in \mathbb{N}_{0}$ and any $\alpha \in \mathbb{R}$ such that $\overline{\mathrm{E}}_{\mathrm{V}}\left(f^{\wedge \ell} \mid s\right)<\alpha$, there is a gamble $h: \Omega \rightarrow \mathbb{N}_{0}$ that is ( $\sup h$ )-measurable such that $m \leq h$ and $\overline{\mathrm{E}}_{\mathrm{V}}\left(f_{h}^{\wedge \ell} \mid s\right) \leq \alpha$. Since $f^{\wedge \ell}$ is both bounded below and above and $\overline{\mathrm{E}}_{\mathrm{V}}$ satisfies $\overline{\nabla 5}$, we have that $\overline{\mathrm{E}}_{\mathrm{V}}\left(f^{\wedge \ell} \mid s\right) \in \mathbb{R}$ and therefore, that $\overline{\mathrm{E}}_{\mathrm{V}}\left(f^{\wedge \ell} \mid s\right)<\overline{\mathrm{E}}_{\mathrm{V}}\left(f^{\wedge \ell} \mid s\right)+1 / \ell$. So in particular, Lemma 34 guarantees that, for any $m \in \mathbb{N}_{0}$, there is a gamble $h: \Omega \rightarrow \mathbb{N}_{0}$ that is (sup $h$ )-measurable such that $m \leq h$ and $\overline{\mathrm{E}}_{\mathrm{V}}\left(f_{h}^{\wedge \ell} \mid s\right) \leq \overline{\mathrm{E}}_{\mathrm{V}}\left(f^{\wedge \ell} \mid s\right)+1 / \ell$. This holds for any $\ell \in \mathbb{N}$, so there is a sequence $\left\{h_{\ell}\right\}_{\ell \in \mathbb{N}_{0}}$ of ( $\sup h_{\ell}$ )-measurable gambles $h_{\ell}: \Omega \rightarrow \mathbb{N}_{0}$ such that $h_{0}=0$ and, for all $\ell \in \mathbb{N}$, satisfies $h_{\ell} \geq \sup h_{\ell-1}+1$ and $\overline{\mathrm{E}}_{\mathrm{V}}\left(f_{h_{\ell}}^{\wedge \ell} \mid s\right) \leq \overline{\mathrm{E}}_{\mathrm{V}}\left(f^{\wedge \ell} \mid s\right)+1 / \ell$. We now show that $\left\{f_{h_{\ell}}^{\wedge \ell}\right\}_{\ell \in \mathbb{N}_{0}}$ is a sequence of finitary gambles that is uniformly bounded below and that converges pointwise to $f$ such that $\lim _{\ell \rightarrow+\infty} \overline{\mathrm{E}}_{\mathrm{V}}\left(f_{h_{\ell}}^{\wedge \ell} \mid s\right)=\overline{\mathrm{E}}_{\mathrm{V}}(f \mid s)$.

Each $f_{h_{\ell}}^{\wedge \ell}$ is a gamble because it is bounded above by $\ell$ and because, since each $f_{n}$ is bounded below by $B$, $f_{h_{\ell}}^{\wedge \ell}$ is bounded below by $\min \{B, \ell\}$. It then also immediately follows that $\left\{f_{h_{\ell}}^{\wedge \ell}\right\}_{\ell \in \mathbb{N}_{0}}$ is uniformly bounded below 
by $\min \{B, 0\}$. Moreover, each $f_{h_{\ell}}^{\wedge \ell}$ is finitary because $h_{\ell}$ is ( $\sup h_{\ell}$ )-measurable. Indeed, the latter implies that $h_{\ell}(\omega)=h_{\ell}(\tilde{\omega})$ for any $\omega \in \Omega$ and any $\tilde{\omega} \in \Gamma\left(\omega^{\left(\sup h_{\ell}\right)}\right)$. Then we also have that

$$
f_{h_{\ell}}^{\wedge \ell}(\omega):=f_{h_{\ell}(\omega)}^{\wedge \ell}(\omega)=f_{h_{\ell}(\tilde{\omega})}^{\wedge \ell}(\omega)=f_{h_{\ell}(\tilde{\omega})}^{\wedge \ell}(\tilde{\omega})=: f_{h_{\ell}}^{\wedge \ell}(\tilde{\omega})
$$

where the third step follows from the fact that $f_{h_{\ell}(\tilde{\omega})}^{\wedge \ell}$ is $h_{\ell}(\tilde{\omega})$-measurable and that $\tilde{\omega} \in \Gamma\left(\omega^{\left(\sup h_{\ell}\right)}\right) \subseteq \Gamma\left(\omega^{h_{\ell}(\tilde{\omega})}\right)$ because $h_{\ell}(\tilde{\omega}) \leq \sup h_{\ell}$. As a consequence, $f_{h_{\ell}}^{\wedge \ell}$ is $\left(\sup h_{\ell}\right)$-measurable too and therefore, finitary.

To see that $\left\{f_{h_{\ell}}^{\wedge \ell}\right\}_{\ell \in \mathbb{N}_{0}}$ converges pointwise to $f$, recall that $\left\{f_{n}\right\}_{n \in \mathbb{N}_{0}}$ is a sequence of gambles that converges

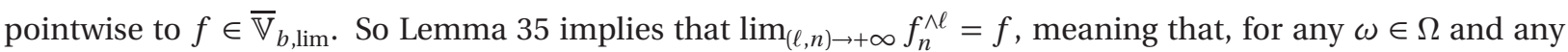
neighbourhood $A$ of $f(\omega)$, there is a couple $\left(\ell^{*}, n^{*}\right) \in \mathbb{N}_{0}^{2}$ such that $f_{n}^{\wedge \ell}(\omega) \in A$ for all $\ell \geq \ell^{*}$ and all $n \geq n^{*}$. Then, since $\left\{h_{\ell}\right\}_{\ell \in \mathbb{N}_{0}}$ is increasing in $\ell$ [because $h_{\ell} \geq \sup h_{\ell-1}+1$ for all $\ell \in \mathbb{N}$ ], there is an $\ell^{\prime} \in \mathbb{N}_{0}$ such that $\ell \geq \ell^{*}$ and $h_{\ell}(\omega) \geq n^{*}$ for all $\ell \geq \ell^{\prime}$. Together with the previous, this implies that $f_{h_{\ell}}^{\wedge \ell}(\omega):=f_{h_{\ell}(\omega)}^{\wedge \ell}(\omega) \in A$ for all $\ell \geq \ell^{\prime}$. Since there is such an $\ell^{\prime} \in \mathbb{N}_{0}$ for any $\omega \in \Omega$ and any neighbourhood $A$ of $f(\omega)$, we have that $\lim _{\ell \rightarrow+\infty} f_{h_{\ell}}^{\wedge \ell}=f$.

Finally, to see that $\lim _{\ell \rightarrow+\infty} \overline{\mathrm{E}}_{\mathrm{V}}\left(f_{h_{\ell}}^{\wedge \ell} \mid s\right)=\overline{\mathrm{E}}_{\mathrm{V}}(f \mid s)$, recall that $\left\{h_{\ell}\right\}_{\ell \in \mathbb{N}_{0}}$ is such that $\overline{\mathrm{E}}_{\mathrm{V}}\left(f_{h_{\ell}}^{\wedge \ell} \mid s\right) \leq \overline{\mathrm{E}}_{\mathrm{V}}\left(f^{\wedge \ell} \mid s\right)+1 / \ell$ for all $\ell \in \mathbb{N}$. So we have that

$$
\limsup _{\ell \rightarrow+\infty} \overline{\mathrm{E}}_{\mathrm{V}}\left(f_{h_{\ell}}^{\wedge \ell} \mid s\right) \leq \limsup _{\ell \rightarrow+\infty}\left(\overline{\mathrm{E}}_{\mathrm{V}}\left(f^{\wedge \ell} \mid s\right)+1 / \ell\right)=\limsup _{\ell \rightarrow+\infty} \overline{\mathrm{E}}_{\mathrm{V}}\left(f^{\wedge \ell} \mid s\right)=\overline{\mathrm{E}}_{\mathrm{V}}(f \mid s)
$$

where the last equality follows from Theorem 23 which we can apply because $\left\{f^{\wedge \ell}\right\}_{\ell \in \mathbb{N}_{0}}$ is a non-decreasing sequence in $\overline{\mathbb{V}}_{b}$ [because $f$ is bounded below] that converges pointwise to $f \in \overline{\mathbb{V}}_{b}$. On the other hand, we have that $\liminf _{\ell \rightarrow+\infty} \overline{\mathrm{E}}_{\mathrm{V}}\left(f_{h_{\ell}}^{\wedge \ell} \mid s\right) \geq \overline{\mathrm{E}}_{\mathrm{V}}(f \mid s)$ because of Corollary25 and the fact that $\left\{f_{h_{\ell}}^{\wedge \ell}\right\}_{\ell \in \mathbb{N}_{0}}$ is uniformly bounded below by $\min \{B, 0\}$ and converges pointwise to $f$. Hence, we conclude that $\lim _{\ell \rightarrow+\infty} \overline{\mathrm{E}}_{\mathrm{V}}\left(f_{h_{\ell}}^{\wedge \ell} \mid s\right)=\overline{\mathrm{E}}_{\mathrm{V}}(f \mid s)$.

Our last step of the proof consists in modifying $\left\{f_{h_{\ell}}^{\wedge \ell}\right\}_{\ell \in \mathbb{N}_{0}}$ such that it becomes a sequence of $n$-measurable gambles that still is uniformly bounded below and converges pointwise to $f$ in such a way that it is continuous with respect to $\overline{\mathrm{E}}_{\mathrm{V}}$. We consider the sequence $\left\{f_{\ell}^{\prime}\right\}_{\ell \in \mathbb{N}_{0}}:=\left\{\left(f_{h_{\ell}}^{\wedge \ell}\right)^{\xi}\right\}_{\ell \in \mathbb{N}_{0}}$ defined through the recursive expression [11, with $c=0$. Then Lemma 29 guarantees that $\left\{f_{\ell}^{\prime}\right\}_{\ell \in \mathbb{N}_{0}}$ is a sequence of $n$-measurable variables. Moreover, from [1], it should be clear that $\left\{f_{\ell}^{\prime}\right\}_{\ell \in \mathbb{N}_{0}}$ is also a sequence of gambles that is uniformly bounded below and that converges pointwise to $f$ such that $\lim _{\ell \rightarrow+\infty} \overline{\mathrm{E}}_{\mathrm{V}}\left(f_{\ell}^{\prime} \mid s\right)=\overline{\mathrm{E}}_{\mathrm{V}}(f \mid s)$. Indeed, in the same way as we have argued in the proof of Theorem 31, this follows from the fact that $\left\{f_{h_{\ell}}^{\wedge \ell}\right\}_{\ell \in \mathbb{N}_{0}}$ is a sequence of gambles that is uniformly bounded below and that converges pointwise to $f$ such that $\lim _{\ell \rightarrow+\infty} \overline{\mathrm{E}}_{\mathrm{V}}\left(f_{h_{\ell}}^{\wedge \ell} \mid s\right)=\overline{\mathrm{E}}_{\mathrm{V}}(f \mid s)$. So we conclude that $\left\{f_{\ell}^{\prime}\right\}_{\ell \in \mathbb{N}_{0}}$ is a sequence of $n$-measurable gambles that is uniformly bounded below and that converges pointwise to $f$ such that $\lim _{\ell \rightarrow+\infty} \overline{\mathrm{E}}_{\mathrm{V}}\left(f_{\ell}^{\prime} \mid s\right)=\overline{\mathrm{E}}_{\mathrm{V}}(f \mid s)$.

\section{Discussion and alternative versions of game-theoretic upper expectations}

An important contribution of this article is that we provide an overview of the main properties that are satisfied by a particular game-theoretic upper expectation operator, for the case where state spaces are assumed to be finite. Some of these properties were already shown to hold in a slightly different setting, and our contribution consists in adapting them to our setting. Other properties, mainly situated in Section 6 and 7 , are entirely 
new and create novel insights. Section 7 for example, shows that the game-theoretic upper expectation $\overline{\mathrm{E}}_{\mathrm{V}}$ behaves in a particularly interesting way with respect to (limits of) finitary variables: Proposition 28 implies that $\overline{\mathrm{E}}_{\mathrm{V}}$ is uniquely characterised by its values on the domain $\overline{\mathbb{V}}_{b, \text { lim }}$, and Theorems 31 and 32 show that $\overline{\mathrm{E}}_{\mathrm{V}}$ has rather strong continuity properties when it comes to sequences of finitary variables.

These results have already proved useful elsewhere, in showing how game-theoretic upper expectations can be alternatively characterised without the use of supermartingales [19]. Moreover, in that same paper [19], we also use these properties to relate the game-theoretic upper expectation to a more traditional measuretheoretic model. Specifically, we show there that game-theoretic and measure-theoretic upper expectations coincide when all local models $\overline{\mathrm{Q}}_{s}$ are precise-that is, when they correspond to linear expectations-and that, in a general imprecise setting, the game-theoretic upper expectation is always more conservative (higher) than the measure-theoretic one. Recently, we discovered that an even stronger relation exists; game-theoretic and measure-theoretic upper expectations coincide on a domain that includes all monotone limits of finitary gambles and all bounded below Borel measurable variables [16]. We also refer to [13, Section 9] for further details on the relation between game-theoretic and measure-theoretic models.

It also becomes apparent, as a consequence of Proposition 2 that Shafer and Vovk's axiomatisation for a local upper expectation can be weakened - and therefore generalised — while leaving the resulting global gametheoretic upper expectation unaffected. This weaker axiomatisation is moreover equivalent to a particular extension of coherence to extended real-valued variables; see Proposition[7 Finally, our axiomatisation, and even Shafer and Vovk's stronger axiomatisation, do not suffice in order to guarantee compatibility of the local models with the global game-theoretic upper expectation. Indeed, it follows from Example1 1 and Corollary 27that such compatibility is only guaranteed if we additionally impose E6 on the local models.

Now, before we conclude the article, we want to clarify why we have chosen to work with the specific gametheoretic upper expectation in Definition 3 As already mentioned in the introduction, it seems appropriate to motivate this, because many slightly different versions of this definition have appeared in the literature 44,12 , 14, 23], and it may perhaps not be entirely clear what these differences entail.

Most of the versions that appear in the literature only differ in how the supermartingales are allowed to behave. In Section 5, it was shown that the definition of $\overline{\mathrm{E}}_{\mathrm{V}}$ is fairly robust with respect to changes that concern the limit behaviour of supermartingales and, more specifically, how this limit behaviour relates to the variable $f$ in consideration: see also $\lfloor 13$, Sections 7-8]. However, there are two issues that do impact the resulting gametheoretic upper expectation, and which were often also considered in the past: whether to define supermartingales as real processes or as extended real processes, and whether they are required to be bounded below or not. Let us first focus on the latter issue.

Considering that supermartingales are interpreted as capital processes, we think it is more natural to assume that they should be bounded below, simply because one cannot borrow an infinite or unbounded amount of money. But there is more to it than this interpretational argument, because by restricting ourselves to bounded below supermartingales we avoid the undesirable situation where the upper expectation would be lower than the lower expectation [4, Example 1]. This would occur irrespectively of whether supermartingales are assumed to be real-valued or extended real-valued. Indeed, let $\overline{\mathbb{M}}$ be the set of all (not necessarily bounded below) extended real-valued supermartingales, let $\overline{\mathbb{M}}^{\prime} \subseteq \overline{\mathbb{M}}$ be the subset of all real-valued supermartingales, and let $\overline{\mathrm{E}}_{\mathrm{ub}}$ and $\overline{\mathrm{E}}_{\mathrm{ub}}^{\prime}$ 
be the game-theoretic upper expectations that are obtained by replacing the set $\overline{\mathbb{M}}_{\mathrm{b}}$ in Definition 3 with the respective sets $\overline{\mathbb{M}}$ and $\overline{\mathbb{M}}^{\prime}$. Since $\overline{\mathbb{M}}^{\prime} \subseteq \overline{\mathbb{M}}$, we clearly have that $\overline{\mathrm{E}}_{\mathrm{ub}}(f \mid s) \leq \overline{\mathrm{E}}_{\mathrm{ub}}^{\prime}(f \mid s)$ and $\underline{\mathrm{E}}_{\mathrm{ub}}^{\prime}(f \mid s) \leq \underline{\mathrm{E}}_{\mathrm{ub}}(f \mid s)$ for all $f \in \overline{\mathbb{V}}$ and all $s \in \mathscr{X}^{*}$. Now, as was shown in [4, Example 1], there is some $f \in \overline{\mathbb{V}}$ and some $s \in \mathscr{X}^{*}$ (and some imprecise probabilities tree) such that $\underline{\mathrm{E}}_{\mathrm{ub}}^{\prime}(f \mid s)>\overline{\mathrm{E}}_{\mathrm{ub}}^{\prime}(f \mid s)$, which then also implies that $\underline{\mathrm{E}}_{\mathrm{ub}}(f \mid s)>\overline{\mathrm{E}}_{\mathrm{ub}}(f \mid s)$. In order to prevent this from happening, we limit ourselves to bounded below (extended real-valued or real-valued) supermartingales.

Now we are left with the question of whether to allow supermartingales to become extended real-valued or not. Let us write $\overline{\mathbb{M}}_{\mathrm{b}}^{\prime}$ to denote the set of all real-valued bounded below supermartingales and let $\overline{\mathrm{E}}_{\mathrm{V}}^{\prime}$ be the corresponding game-theoretic upper expectation:

$$
\overline{\mathrm{E}}_{\mathrm{V}}^{\prime}(f \mid s):=\inf \left\{\mathscr{M}(s): \mathscr{M} \in \overline{\mathbb{M}}_{\mathrm{b}}^{\prime} \text { and } \liminf \mathscr{M} \geq_{s} f\right\} \text { for all } f \in \overline{\mathbb{V}} \text { and all } s \in \mathscr{X}^{*} .
$$

At first sight, we would be inclined to use the game-theoretic upper expectation $\overline{\mathrm{E}}_{\mathrm{V}}^{\prime}$ with real-valued supermartingales, because it allows for a more direct interpretation. Indeed, if we interpret a supermartingale as the possible evolution of a person's capital, it is not clear to us what it means if this person's capital were to become infinite in value. Moreover, on the domain $\mathbb{V} \times \mathscr{X}^{*}$, the version $\overline{\mathrm{E}}_{\mathrm{V}}^{\prime}$ with real supermartingales has rather desirable properties; as we will show below with Proposition 36, it coincides with our version $\overline{\mathrm{E}}_{\mathrm{V}}$ on the domain $\mathbb{V} \times \mathscr{X}^{*}$. All things considered, it ought not to surprise that $\overline{\mathrm{E}}_{\mathrm{V}}^{\prime}$ was frequently adopted in earlier work by both ourselves [4, 20] and by Shafer and Vovk [12, 23]. However, as we will soon point out, it has a rather undesirable property once it is applied to the entire domain $\overline{\mathbb{V}} \times \mathscr{X}^{*}$.

Before we do so, we want to draw the attention to the fact that there is yet another, maybe even more intuitive, possible way of defining game-theoretic upper expectations: using bounded (above and below) real-valued supermartingales. On the one hand, as we already explained, we consider it sensible to assume that supermartingales should be bounded below because one cannot borrow an infinite or unbounded amount of money. On the other hand, for similar reasons, we could also impose that supermartingales should be bounded above; receiving an infinite or unbounded amount of money seems impossible in practice too. We therefore think it is appropriate to also consider a definition of the game-theoretic upper expectation with bounded (and hence real-valued) supermartingales. Let $\overline{\mathbb{M}}_{\mathrm{b}}^{\prime \prime}$ be the set of all such bounded (real-valued) supermartingales and let $\overline{\mathrm{E}}_{\mathrm{V}}^{\prime \prime}$ be the corresponding game-theoretic upper expectation:

$$
\overline{\mathrm{E}}_{\mathrm{V}}^{\prime \prime}(f \mid s):=\inf \left\{\mathscr{M}(s): \mathscr{M} \in \overline{\mathbb{M}}_{\mathrm{b}}^{\prime \prime} \text { and } \liminf \mathscr{M} \geq_{s} f\right\} \text { for all } f \in \overline{\mathbb{V}} \text { and all } s \in \mathscr{X}^{*} .
$$

Despite that it allows for a rather direct interpretation, such a version $\bar{E}_{V}^{\prime \prime}$ of the game-theoretic upper expectation has, to the best of our knowledge, never been used in the literature. The reason, presumably, is that for extended real variables $f$ that are not bounded above, the value of $\overline{\mathrm{E}}_{\mathrm{V}}^{\prime \prime}(f)$ does not provide any information because it will simply be equal to $+\infty$; that is, the infimum over the empty set. However, as our next result shows, this version $\overline{\mathrm{E}}_{\mathrm{V}}^{\prime \prime}$ coincides with our version $\overline{\mathrm{E}}_{\mathrm{V}}$-and therefore also with the version $\overline{\mathrm{E}}_{\mathrm{V}}^{\prime}$ that uses real-valued bounded below supermartingales—on the domain $\mathbb{V} \times \mathscr{X}^{*}$ of all gambles (and all situations).

Proposition 36. For any gamble $f \in \mathbb{V}$ and any situation $s \in \mathscr{X}^{*}$, we have that $\overline{\mathrm{E}}_{\mathrm{V}}(f \mid s)=\overline{\mathrm{E}}_{\mathrm{V}}^{\prime}(f \mid s)=\overline{\mathrm{E}}_{\mathrm{V}}^{\prime \prime}(f \mid s)$.

This result relies on the following two lemmas. 
Lemma 37. For any $\mathscr{M} \in \overline{\mathbb{M}}_{\mathrm{b}}$ and any $B \in \mathbb{R}$, the process $\mathscr{M}_{B}$, defined by $\mathscr{M}_{B}(s):=\min \{\mathscr{M}(s), B\}$ for all $s \in \mathscr{X}^{*}$, is a bounded real-valued supermartingale.

Proof. It is clear that, since $\mathscr{M}$ is a bounded below extended real process, $\mathscr{M}_{B}$ is a bounded (above and below) real process. Moreover, $\mathscr{M}_{B}(s) \leq \mathscr{M}(s)$ for all $s \in \mathscr{X}^{*}$, so it follows that $\mathscr{M}_{B}(s \cdot) \leq \mathscr{M}(s \cdot)$ for all $s \in \mathscr{X}^{*}$. Fix any $s \in \mathscr{X}^{*}$. If $\mathscr{M}(s) \leq B$, then

$$
\overline{\mathrm{Q}}_{s}\left(\mathscr{M}_{B}(s \cdot)\right) \leq \overline{\mathrm{Q}}_{s}(\mathscr{M}(s \cdot)) \leq \mathscr{M}(s)=\mathscr{M}_{B}(s)
$$

where the first equality follows from the monotonicity [E4] of $\overline{\mathrm{Q}}_{s}$ (and the fact that $\mathscr{M}\left(s \cdot\right.$ ) and $\mathscr{M}_{B}(s \cdot$ ) are bounded below). If $\mathscr{M}(s)>B$, it follows from $\mathscr{M}_{B}(s \cdot) \leq B$ and the monotonicity [E4] of $\overline{\mathrm{Q}}_{s}$ that

$$
\overline{\mathrm{Q}}_{s}\left(\mathscr{M}_{B}(s \cdot)\right) \leq \overline{\mathrm{Q}}_{s}(B) \stackrel{\text { 䒰 }}{=} B=\mathscr{M}_{B}(s) .
$$

So, we conclude that $\overline{\mathrm{Q}}_{s}\left(\mathscr{M}_{B}(s \cdot)\right) \leq \mathscr{M}_{B}(s)$ for all situations $s \in \mathscr{X}^{*}$. Hence, $\mathscr{M}_{B}$ is a bounded real-valued supermartingale.

Lemma 38. For any extended real process $\mathscr{P}$ and any path $\omega \in \Omega$, we have that

$$
\min \left\{B, \liminf _{n \rightarrow+\infty} \mathscr{P}\left(\omega^{n}\right)\right\}=\liminf _{n \rightarrow+\infty} \min \left\{B, \mathscr{P}\left(\omega^{n}\right)\right\} \text { for all } B \in \mathbb{R}
$$

Proof. Fix any $B \in \mathbb{R}$. It is easy to check that $\min \left\{B, \liminf _{n \rightarrow+\infty} \mathscr{P}\left(\omega^{n}\right)\right\} \geq \liminf _{n \rightarrow+\infty} \min \left\{B, \mathscr{P}\left(\omega^{n}\right)\right\}$. We prove the converse inequality by contradiction. Suppose that

$$
\min \left\{B, \liminf _{n \rightarrow+\infty} \mathscr{P}\left(\omega^{n}\right)\right\}>\liminf _{n \rightarrow+\infty} \min \left\{B, \mathscr{P}\left(\omega^{n}\right)\right\}
$$

or, equivalently, that

$$
\min \left\{B, \liminf _{n \rightarrow+\infty} \mathscr{P}\left(\omega^{n}\right)\right\}>\sup _{m} \inf _{n \geq m} \min \left\{B, \mathscr{P}\left(\omega^{n}\right)\right\} .
$$

Then there is some $\epsilon \in \mathbb{R}_{>0}$ such that

$$
\min \left\{B, \liminf _{n \rightarrow+\infty} \mathscr{P}\left(\omega^{n}\right)\right\}-\epsilon>\inf _{n \geq m} \min \left\{B, \mathscr{P}\left(\omega^{n}\right)\right\}=\min \left\{B, \inf _{n \geq m} \mathscr{P}\left(\omega^{n}\right)\right\}
$$

for all $m \in \mathbb{N}_{0}$. Since $\min \left\{B, \liminf _{n \rightarrow+\infty} \mathscr{P}\left(\omega^{n}\right)\right\}-\epsilon<B$, it can only be that

$$
\inf _{n \geq m} \mathscr{P}\left(\omega^{n}\right)<\min \left\{B, \liminf _{n \rightarrow+\infty} \mathscr{P}\left(\omega^{n}\right)\right\}-\epsilon \leq \liminf _{n \rightarrow+\infty} \mathscr{P}\left(\omega^{n}\right)-\epsilon \text { for all } m \in \mathbb{N}_{0},
$$

from which we infer that $\inf _{n \geq m} \mathscr{P}\left(\omega^{n}\right)<\sup _{k} \inf _{n \geq k} \mathscr{P}\left(\omega^{n}\right)-\epsilon$ for all $m \in \mathbb{N}_{0}$. This contradicts the definition of the supremum operator.

Proof of Proposition 36, Since $\overline{\mathbb{M}}_{\mathrm{b}}^{\prime \prime} \subseteq \overline{\mathbb{M}}_{\mathrm{b}}^{\prime} \subseteq \overline{\mathbb{M}}_{\mathrm{b}}$, it is clear that $\overline{\mathrm{E}}_{\mathrm{V}}(f \mid s) \leq \overline{\mathrm{E}}_{\mathrm{V}}^{\prime}(f \mid s) \leq \overline{\mathrm{E}}_{\mathrm{V}}^{\prime \prime}(f \mid s)$. So it suffices to prove that $\overline{\mathrm{E}}_{\mathrm{V}}^{\prime \prime}(f \mid s) \leq \overline{\mathrm{E}}_{\mathrm{V}}(f \mid s)$. Consider any $\mathscr{M} \in \overline{\mathrm{M}}_{\mathrm{b}}$ such that liminf $\mathscr{M} \geq_{s} f$. Let $B:=\sup f$ and let $\mathscr{M}_{B}$ be defined by $\mathscr{M}_{B}(t):=\min \{\mathscr{M}(t), B\}$ for all $t \in \mathscr{X}^{*}$. Since $f$ is a gamble, $\sup f$ is real and hence, due to Lemma 37, $\mathscr{M}_{B}$ is a bounded real-valued supermartingale. Moreover, we have that $B \geq f$ and $\liminf \mathscr{M}_{s} f$, and therefore that $\min \{B, \liminf \mathscr{M}\} \geq_{s} f$, which by Lemma 38 implies that $\liminf \mathscr{M}_{B} \geq_{s} f$. Together with the fact that $\mathscr{M}_{B} \in \overline{\mathbb{M}}_{\mathrm{b}}^{\prime \prime}$, 
this implies by the definition of $\overline{\mathrm{E}}_{\mathrm{V}}^{\prime \prime}$ that $\overline{\mathrm{E}}_{\mathrm{V}}^{\prime \prime}(f \mid s) \leq \mathscr{M}_{B}(s) \leq \mathscr{M}(s)$. Since this holds for any $\mathscr{M} \in \overline{\mathrm{M}}_{\mathrm{b}}$ such that $\liminf \mathscr{M} \geq_{s} f$, we infer from the definition of $\overline{\mathrm{E}}_{\mathrm{V}}$ that $\overline{\mathrm{E}}_{\mathrm{V}}^{\prime \prime}(f \mid s) \leq \overline{\mathrm{E}}_{\mathrm{V}}(f \mid s)$.

So the versions $\overline{\mathrm{E}}_{\mathrm{V}}, \overline{\mathrm{E}}_{\mathrm{V}}^{\prime}$ and $\overline{\mathrm{E}}_{\mathrm{V}}^{\prime \prime}$ are all mathematically equivalent on $\mathbb{V} \times \mathscr{X}^{*}$. We therefore prefer $\overline{\mathrm{E}}_{\mathrm{V}}^{\prime \prime}$ over both $\overline{\mathrm{E}}_{\mathrm{V}}$ and $\overline{\mathrm{E}}_{\mathrm{V}}^{\prime}$ on $\mathbb{V} \times \mathscr{X}^{*}$ because it can be given a (more) direct interpretation. However, if we consider the entire domain $\overline{\mathbb{V}} \times \mathscr{X}^{*}$, then the version $\overline{\mathrm{E}}_{\mathrm{V}}^{\prime \prime}$ is unsuitable because, as explained above, it assigns $+\infty$ to every variable that is not bounded above. Our remaining two options are then to use either $\overline{\mathrm{E}}_{\mathrm{V}}$ or $\overline{\mathrm{E}}_{\mathrm{V}}^{\prime}$. From a purely interpretational point of view, we prefer $\overline{\mathrm{E}}_{\mathrm{V}}^{\prime}$. Unfortunately, the version $\overline{\mathrm{E}}_{\mathrm{V}}^{\prime}$-and also the version $\overline{\mathrm{E}}_{\mathrm{V}}^{\prime \prime}$-does not satisfy continuity with respect to non-decreasing sequences, which we consider to be an important mathematical drawback.

Example 2. Consider an uncertain process with state space $\mathscr{X}:=\{0,1\}$ and let $\overline{\mathrm{Q}}_{\square}(f):=f(0)$ for all $f \in \overline{\mathscr{L}}(\mathscr{X})$. It can easily be checked that $\overline{\mathrm{Q}}_{\square}$ satisfies properties $\mathrm{E1} \rightleftarrows \mathrm{E} 5$, For any $n \in \mathbb{N}$, the variable $n \mathbb{I}_{1}\left(X_{1}\right)$ is a gamble, so Proposition[36implies that $\overline{\mathrm{E}}_{\mathrm{V}}\left(n \mathbb{I}_{1}\left(X_{1}\right)\right)=\overline{\mathrm{E}}_{\mathrm{V}}^{\prime}\left(n \mathbb{I}_{1}\left(X_{1}\right)\right)=\overline{\mathrm{E}}_{\mathrm{V}}^{\prime \prime}\left(n \mathbb{I}_{1}\left(X_{1}\right)\right)$. Then, taking into account the boundedness of $n \mathbb{I}_{1}\left(X_{1}\right)$, it follows from Proposition [14 that $\overline{\mathrm{E}}_{\mathrm{V}}^{\prime \prime}\left(n \mathbb{I}_{1}\left(X_{1}\right)\right)=\overline{\mathrm{E}}_{\mathrm{V}}^{\prime}\left(n \mathbb{I}_{1}\left(X_{1}\right)\right)=\overline{\mathrm{E}}_{\mathrm{V}}\left(n \mathbb{I}_{1}\left(X_{1}\right)\right)=\overline{\mathrm{Q}}_{\square}\left(n \mathbb{I}_{1}\right)$, which by definition of $\overline{\mathrm{Q}}_{\square}$ leads us to conclude that $\overline{\mathrm{E}}_{\mathrm{V}}^{\prime \prime}\left(n \mathbb{I}_{1}\left(X_{1}\right)\right)=\overline{\mathrm{E}}_{\mathrm{V}}^{\prime}\left(n \mathbb{I}_{1}\left(X_{1}\right)\right)=n \mathbb{I}_{1}(0)=0$ for all $n \in \mathbb{N}$.

On the other hand, consider the upper expectation $\overline{\mathrm{E}}_{\mathrm{V}}^{\prime}\left(+\infty \mathbb{I}_{1}\left(X_{1}\right)\right)$. It is defined as the infimum of $\mathscr{M}(\square)$ over real-valued supermartingales $\mathscr{M} \in \overline{\mathbb{M}}_{\mathrm{b}}^{\prime}$ such that $\liminf \mathscr{M} \geq+\infty \mathbb{I}_{1}\left(X_{1}\right)$. Any such supermartingale $\mathscr{M}$ should converge to $+\infty$ on all paths $\omega \in \Gamma(1)$ such that $\omega^{1}=1$. Since $\mathscr{M} \in \overline{\mathbb{M}}_{\mathrm{b}}^{\prime} \subseteq \overline{\mathbb{M}}_{\mathrm{b}}$, we can then apply Lemma 11] to infer that $\mathscr{M}(1)=+\infty$. This contradicts the real-valuedness of $\mathscr{M}$, which allows us to conclude that there is no $\mathscr{M} \in \overline{\mathbb{M}}_{\mathrm{b}}^{\prime}$ such that liminf $\mathscr{M} \geq+\infty \mathbb{I}_{1}\left(X_{1}\right)$. Hence, $\overline{\mathrm{E}}_{\mathrm{V}}^{\prime}\left(+\infty \mathbb{I}_{1}\left(X_{1}\right)\right)=+\infty$ and therefore, because $\overline{\mathrm{E}}_{\mathrm{V}}^{\prime}\left(+\infty \mathbb{I}_{1}\left(X_{1}\right)\right) \leq$ $\overline{\mathrm{E}}_{\mathrm{V}}^{\prime \prime}\left(+\infty \mathbb{I}_{1}\left(X_{1}\right)\right)$ (recall that $\left.\overline{\mathrm{M}}_{\mathrm{b}}^{\prime \prime} \subseteq \overline{\mathrm{M}}_{\mathrm{b}}^{\prime}\right)$, also $\overline{\mathrm{E}}_{\mathrm{V}}^{\prime \prime}\left(+\infty \mathbb{I}_{1}\left(X_{1}\right)\right)=+\infty$.

All together, we have that $\lim _{n \rightarrow+\infty} n \mathbb{I}_{1}\left(X_{1}\right)=+\infty \mathbb{I}_{1}\left(X_{1}\right)$ but

$$
\lim _{n \rightarrow+\infty} \overline{\mathrm{E}}_{\mathrm{V}}^{\prime}\left(n \mathbb{I}_{1}\left(X_{1}\right)\right) \neq \overline{\mathrm{E}}_{\mathrm{V}}^{\prime}\left(+\infty \mathbb{I}_{1}\left(X_{1}\right)\right) \text { and } \lim _{n \rightarrow+\infty} \overline{\mathrm{E}}_{\mathrm{V}}^{\prime \prime}\left(n \mathbb{I}_{1}\left(X_{1}\right)\right) \neq \overline{\mathrm{E}}_{\mathrm{V}}^{\prime \prime}\left(+\infty \mathbb{I}_{1}\left(X_{1}\right)\right) \text {, }
$$

which implies that both $\overline{\mathrm{E}}_{\mathrm{V}}^{\prime}$ and $\overline{\mathrm{E}}_{\mathrm{V}}^{\prime \prime}$ fail to satisfy continuity with respect to non-decreasing sequences.

We have chosen to work with the version $\overline{\mathrm{E}}_{\mathrm{V}}$ in this paper, and therefore implicitly assumed that global (gametheoretic) upper expectations satisfy continuity with respect to non-decreasing sequences. This is in line with how we introduced our local uncertainty models $\overline{\mathrm{Q}}_{s}$ : the continuity propertyE5 was directly adopted as part of their definition. The fact that our global upper expectation $\bar{E}_{\mathrm{V}}$ (and each local upper expectation) satisfies this non-decreasing continuity is mathematically convenient, but it does require a definition that is more abstract compared to $\overline{\mathrm{E}}_{\mathrm{V}}^{\prime}$ or $\overline{\mathrm{E}}_{\mathrm{V}}^{\prime \prime}$. Nonetheless, we do not regard this as an issue because, on the domain $\mathbb{V} \times \mathscr{X}^{*}$, our version $\overline{\mathrm{E}}_{\mathrm{V}}$ is equivalent to the version $\overline{\mathrm{E}}_{\mathrm{V}}^{\prime \prime}$ (or $\overline{\mathrm{E}}_{\mathrm{V}}^{\prime}$ ). So one could just as well consider it to be defined as $\overline{\mathrm{E}}_{\mathrm{V}}^{\prime \prime}$ (or $\overline{\mathrm{E}}_{\mathrm{V}}^{\prime}$ ) on this restricted domain. Then, as far as the values of $\overline{\mathrm{E}}_{\mathrm{V}}$ on $(\overline{\mathbb{V}} \backslash \mathbb{V}) \times \mathscr{X}^{*}$ are concerned, recall that these are uniquely determined by the values on $\mathbb{V} \times \mathscr{X}^{*}$ through the continuity of $\overline{\mathrm{E}}_{\mathrm{V}}$; see the discussion below Proposition 26. Hence, we can regard $\overline{\mathrm{E}}_{\mathrm{V}}$ on $(\overline{\mathbb{V}} \backslash \mathbb{V}) \times \mathscr{X}^{*}$ as an extension of $\overline{\mathrm{E}}_{\mathrm{V}}$ on $\mathbb{V} \times \mathscr{X}^{*}$, where the latter is justified by its equivalence with $\overline{\mathrm{E}}_{\mathrm{V}}^{\prime \prime}$, and where the extension is motivated by assumptions of continuity. 


\section{Acknowledgements}

Natan T'Joens's research was supported and funded by the Special Research Fund (BOF) of Ghent University (reference number: 356). The research by Jasper De Bock and Gert de Cooman was funded through project number 3G028919 of the Research Foundation - Flanders (FWO). We are also indebted to an anonymous reviewer who provided helpful feedback.

\section{References}

[1] T. Augustin, F. Coolen, G. de Cooman, and M. Troffaes. Introduction to Imprecise Probabilities. Wiley, Chichester, 2014.

[2] P. Billingsley. Probability and Measure. Wiley, New York, 1995.

[3] J. De Bock and G. de Cooman. Continuity of imprecise stochastic processes with respect to the pointwise convergence of monotone sequences. arXiv:1402.3056, 2014.

[4] G. de Cooman, J. De Bock, and S. Lopatatzidis. Imprecise stochastic processes in discrete time: global models, imprecise Markov chains, and ergodic theorems. International Journal of Approximate Reasoning, 76:18-46, 2016.

[5] G. de Cooman and F. Hermans. Imprecise probability trees: Bridging two theories of imprecise probability. Artificial Intelligence, 172:1400-1427, 2008.

[6] G. de Cooman, F. Hermans, and E. Quaeghebeur. Imprecise Markov chains and their limit behaviour. Probability in the Engineering and Informational Sciences, 23(4):597-635, 2009.

[7] G. de Cooman and M. Troffaes. Lower Previsions. Wiley, Chichester, 2014.

[8] F. Hermans and D. Škulj. Stochastic Processes. In T. Augustin, F. Coolen, G. de Cooman, and M. Troffaes, editors, Introduction to Imprecise Probabilities, pages 258-278. Wiley, Chichester, 2014.

[9] T. Krak, N. T'Joens, and J. De Bock. Hitting times and probabilities for imprecise Markov chains. In Proceedings of the 11th International Symposium on Imprecise Probabilities: Theories and Applications, volume 103, pages 265-275, 2019.

[10] S. Lopatatzidis. Robust Modelling and Optimisation in Stochastic Processes using Imprecise Probabilities, with an Application to Queueing Theory. PhD thesis, Ghent University, 2017.

[11] E. Quaeghebeur, G. de Cooman, and F. Hermans. Accept \& reject statement-based uncertainty models. International Journal of Approximate Reasoning, 57:69-102, 2015.

[12] G. Shafer and V. Vovk. Probability and Finance: It's Only a Game! Wiley, New York, 2001.

[13] G. Shafer and V. Vovk. Game-Theoretic Foundations for Probability and Finance. Wiley, Hoboken, New Jersey, 2019.

[14] G. Shafer, V. Vovk, and A. Takemura. Lévy's zero-one law in game-theoretic probability. Journal of Theoretical Probability, 25:1-24, 2012.

[15] A.N. Shiryaev. Probability. Springer-Verlag, New York, 1996.

[16] N. T'Joens and J. De Bock. Global upper expectations for discrete-time stochastic processes: In practice, they are all the same! Extended version of a contribution submitted for possible publication in the Proceedings of ISIPTA 2021, see ArXiv:2102.13075.

[17] N. T'Joens, J. De Bock, and G. de Cooman. Continuity properties of game-theoretic upper expectations. arXiv:1902.09406, 2019.

[18] N. T'Joens, J. De Bock, and G. de Cooman. In search of a global belief model for discrete-time uncertain processes. In Proceedings of the 11th International Symposium on Imprecise Probabilities: Theories and Applications, volume 103, pages 377-385, 2019.

[19] N. T'Joens, J. De Bock, and G. de Cooman. A particular upper expectation as global belief model for discrete-time finite-state uncertain processes. International Journal of Approximate Reasoning, 131:30 - 55, 2021.

[20] N. T'Joens, G. de Cooman, and J. De Bock. Continuity of the Shafer-Vovk-Ville operator. In Proceedings of the 9th International Conference on Soft Methods in Probability and Statistics, volume 832, pages 200-207, 2018.

[21] N. T'Joens, T. Krak, J. De Bock, and G. de Cooman. A recursive algorithm for computing inferences in imprecise Markov chains. In Proceedings of the 15th European Conference on Symbolic and Quantitative Approaches to Reasoning with Uncertainty, volume 11726, pages 455-465, 2019.

[22] J. Ville. Étude critique de la notion de collectif. Thèses françaises de l'entre-deux-guerres. Numdam, 1939.

[23] V. Vovk and G. Shafer. Game-theoretic Probability. In T. Augustin, F. Coolen, G. de Cooman, and M. Troffaes, editors, Introduction to Imprecise Probabilities, pages 114-134. Wiley, Chichester, 2014.

[24] P. Walley. Statistical Reasoning with Imprecise Probabilities. Chapman and Hall, London, 1991. 


\section{Appendix A. Proofs of the results in Section[5}

In the following proofs, we will frequently use the terminology and notations concerning cuts, as introduced in the paragraph above Lemma 34. Moreover, for any two cuts $U$ and $V$, we will write $U \sqsubset V$ if $(\forall v \in V)(\exists u \in$ $U) u \sqsubset v$, and similarly for the relations $\sqsubseteq, \sqsupset$ and $\sqsupseteq$. We will also consider the following sets:

$$
\begin{aligned}
& {[U, V]:=\left\{s \in \mathscr{X}^{*}:(\exists u \in U)(\exists v \in V) u \sqsubseteq s \sqsubseteq v\right\},} \\
& {[U, V):=\left\{s \in \mathscr{X}^{*}:(\exists u \in U)(\exists v \in V) u \sqsubseteq s \sqsubset v\right\},} \\
& (U, V]:=\left\{s \in \mathscr{X}^{*}:(\exists u \in U)(\exists v \in V) u \sqsubset s \sqsubseteq v\right\}, \\
& (U, V):=\left\{s \in \mathscr{X}^{*}:(\exists u \in U)(\exists v \in V) u \sqsubset s \sqsubset v\right\} .
\end{aligned}
$$

Proof of Proposition 17, Let $t \in \mathscr{X}^{*}$ be any fixed situation where $\mathscr{M}(t)$ is real. We can assume that $\mathscr{M}$ is nonnegative and that $\mathscr{M}(t)=1$ without loss of generality. Indeed, because the original supermartingale is bounded below and real in $t$, we can obtain such a process by translating and scaling — by adding a positive constant and then multiplying the supermartingale by a positive real—-the originally considered supermartingale in an appropriate way. This process will then again be a (bounded below) supermartingale because the local models $\overline{\mathrm{Q}}_{s}$ satisfyE8 andE9 Moreover, the new supermartingale will have the same convergence character as the original one.

To start, fix any couple of rational numbers $0<a<b$ and consider the following recursively constructed sequences of cuts $\left\{U_{k}^{a, b}\right\}_{k \in \mathbb{N}}$ and $\left\{V_{k}^{a, b}\right\}_{k \in \mathbb{N}}$. Let $V_{1}^{a, b}:=\left\{s \sqsupseteq t: \mathscr{M}(s)<a\right.$ and $\left.\left(\forall s^{\prime} \in[t, s)\right) \mathscr{M}\left(s^{\prime}\right) \geq a\right\}$ and, for $k \in \mathbb{N}$,

1. let $U_{k}^{a, b}:=\left\{s \in \mathscr{X}^{*}: V_{k}^{a, b} \sqsubset s: \mathscr{M}(s)>b\right.$ and $\left.\left(\forall s^{\prime} \in\left(V_{k}^{a, b}, s\right)\right) \mathscr{M}\left(s^{\prime}\right) \leq b\right\}$;

2. let $V_{k+1}^{a, b}:=\left\{s \in \mathscr{X}^{*}: U_{k}^{a, b} \sqsubset s, \mathscr{M}(s)<a\right.$ and $\left.\left(\forall s^{\prime} \in\left(U_{k}^{a, b}, s\right)\right) \mathscr{M}\left(s^{\prime}\right) \geq a\right\}$.

Note that all $U_{k}^{a, b}$ and all $V_{k}^{a, b}$ are indeed (partial or complete) cuts.

Next, consider the extended real process $\mathscr{M}^{a, b}$ defined by $\mathscr{M}^{a, b}(s):=\mathscr{M}(t)$ for all $s \not \supset t$ and by

$$
\mathscr{M}^{a, b}(s x):= \begin{cases}\mathscr{M}^{a, b}(s)+[\mathscr{M}(s x)-\mathscr{M}(s)] & \text { if } s \in\left[V_{k}^{a, b}, U_{k}^{a, b}\right) \text { for some } k \in \mathbb{N} ; \\ \mathscr{M}^{a, b}(s) & \text { otherwise, }\end{cases}
$$

for all $s x \sqsupset t$ with $x \in \mathscr{X}$. We prove that $\mathscr{M}^{a, b}$ is a non-negative supermartingale that converges to $+\infty$ on all paths $\omega \in \Gamma(t)$ such that

$$
\liminf \mathscr{M}(\omega)<a<b<\limsup \mathscr{M}(\omega) .
$$

For any situation $s$ and for any $k \in \mathbb{N}$, when $U_{k}^{a, b} \sqsubset s$, we denote by $u_{k}^{s}$ the (necessarily unique) situation in $U_{k}^{a, b}$ such that $u_{k}^{s} \sqsubset s$. Similarly, for any $k \in \mathbb{N}$, when $V_{k}^{a, b} \sqsubset s$, we denote by $v_{k}^{s}$ the (necessarily unique) situation in $V_{k}^{a, b}$ such that $v_{k}^{s} \sqsubset s$. Note that $V_{1}^{a, b} \sqsubset U_{1}^{a, b} \sqsubset V_{2}^{a, b} \sqsubset \cdots \sqsubset V_{n}^{a, b} \sqsubset U_{n}^{a, b} \sqsubset \cdots$. Hence, for any situation $s$ we can distinguish the following three cases:

- The first case is that $V_{1}^{a, b} \not \subset s$. Then we have that

$$
\mathscr{M}^{a, b}(s)=\mathscr{M}^{a, b}(t)=\mathscr{M}(t)=1 .
$$


- The second case is that $V_{k}^{a, b} \sqsubset s$ and $U_{k}^{a, b} \not \subset s$ for some $k \in \mathbb{N}$. Then by applying Equation A.1 for each subsequent step and cancelling out the intermediate terms, which is possible because $\mathscr{M}$ is real for any situation $s^{\prime} \in \mathscr{X}^{*}$ such that $V_{k^{\prime}}^{a, b} \sqsubseteq s^{\prime}$ and $U_{k^{\prime}}^{a, b} \nsubseteq s^{\prime}$ for some $k^{\prime} \in \mathbb{N}$ [this follows readily from the definition of the cuts $V_{k^{\prime}}^{a, b}$ and $\left.U_{k^{\prime}}^{a, b}\right]$, we have that

$$
\mathscr{M}^{a, b}(s)-\mathscr{M}^{a, b}(t)=\sum_{\ell=1}^{k-1}\left[\mathscr{M}\left(u_{\ell}^{s}\right)-\mathscr{M}\left(v_{\ell}^{s}\right)\right]+\mathscr{M}(s)-\mathscr{M}\left(v_{k}^{s}\right)
$$

- The third case is that $U_{k}^{a, b} \sqsubset s$ and $V_{k+1}^{a, b} \not \subset s$ for some $k \in \mathbb{N}$. Then we have that

$$
\mathscr{M}^{a, b}(s)-\mathscr{M}^{a, b}(t)=\sum_{\ell=1}^{k}\left[\mathscr{M}\left(u_{\ell}^{s}\right)-\mathscr{M}\left(v_{\ell}^{s}\right)\right]
$$

where, again, we used the fact that $\mathscr{M}$ is real for any situation $s^{\prime} \in \mathscr{X}^{*}$ such that $V_{k^{\prime}}^{a, b} \sqsubseteq s^{\prime}$ and $U_{k^{\prime}}^{a, b} \nsubseteq s^{\prime}$ for some $k^{\prime} \in \mathbb{N}$.

That $\mathscr{M}^{a, b}(s)$ is non-negative, is trivially satisfied in the first case because $\mathscr{M}$ is non-negative. To see that this is also true for the third case, observe that $0<b<\mathscr{M}\left(u_{\ell}^{s}\right)$ and $0 \leq \mathscr{M}\left(v_{\ell}^{s}\right)<a$ for all $\ell \in\{1, \ldots, k\}$. This implies that $\mathscr{M}\left(u_{\ell}^{s}\right)-\mathscr{M}\left(v_{\ell}^{s}\right)>b-a>0$ for all $\ell \in\{1, \ldots, k\}$ and therefore directly that $\mathscr{M}^{a, b}(s)$ is non-negative because of Equation $\overline{A .5}$ and the fact that $\mathscr{M}^{a, b}(t)=\mathscr{M}(t)$ is non-negative. In the second case, it follows from Equations (A.3), A.4) and A.5 that

$$
\mathscr{M}^{a, b}(s)=\mathscr{M}^{a, b}\left(v_{k}^{s}\right)+\mathscr{M}(s)-\mathscr{M}\left(v_{k}^{s}\right) .
$$

We prove by induction that $\mathscr{M}^{a, b}\left(v_{\ell}^{s}\right) \geq \mathscr{M}\left(v_{\ell}^{s}\right)$ for all $\ell \in\{1, \ldots, k\}$, and therefore, by Equation (A.6) and because $\mathscr{M}$ is non-negative, that $\mathscr{M}^{a, b}(s)$ is non-negative.

If $\ell=1$, then either $v_{1}^{s}=t$ or $v_{1}^{s} \neq t$. If $v_{1}^{s}=t$, then $\mathscr{M}^{a, b}\left(v_{1}^{s}\right)=\mathscr{M}^{a, b}(t)=\mathscr{M}(t)=\mathscr{M}\left(v_{1}^{s}\right)$. If $v_{1}^{s} \neq t$, we have, by the definition of $V_{1}^{a, b}$, that $\mathscr{M}\left(v_{1}^{s}\right)<a$ and $a \leq \mathscr{M}(t)=\mathscr{M}^{a, b}(t)=\mathscr{M}^{a, b}\left(v_{1}^{s}\right)$. Hence, in both cases, we have that $\mathscr{M}^{a, b}\left(v_{1}^{s}\right) \geq \mathscr{M}\left(v_{1}^{s}\right)$. Now suppose that $\mathscr{M}^{a, b}\left(v_{\ell}^{s}\right) \geq \mathscr{M}\left(v_{\ell}^{s}\right)$ for some $\ell \in\{1, \ldots, k-1\}$. Then, $\mathscr{M}^{a, b}\left(v_{\ell+1}^{s}\right)=$ $\mathscr{M}^{a, b}\left(v_{\ell}^{s}\right)+\left[\mathscr{M}\left(u_{\ell}^{s}\right)-\mathscr{M}\left(v_{\ell}^{s}\right)\right] \geq \mathscr{M}\left(u_{\ell}^{s}\right)>b>a>\mathscr{M}\left(v_{\ell+1}^{s}\right)$, which concludes our induction step. So indeed $\mathscr{M}^{a, b}\left(v_{\ell}^{s}\right) \geq \mathscr{M}\left(v_{\ell}^{s}\right)$ for all $\ell \in\{1, \ldots, k\}$.

Next, we show that $\overline{\mathrm{Q}}_{s}\left(\mathscr{M}^{a, b}(s \cdot)\right) \leq \mathscr{M}^{a, b}(s)$ for all $s \in \mathscr{X}^{*}$, and hence, that $\mathscr{M}$ is a non-negative supermartingale. Consider any $s \in \mathscr{X}^{*}$. If $s \in\left[V_{k}^{a, b}, U_{k}^{a, b}\right)$ for some $k \in \mathbb{N}$, it follows from Equation A.1] that

$$
\overline{\mathrm{Q}}_{s}\left(\mathscr{M}^{a, b}(s \cdot)\right)=\overline{\mathrm{Q}}_{s}\left(\mathscr{M}^{a, b}(s)+\mathscr{M}(s \cdot)-\mathscr{M}(s)\right) \stackrel{\mathrm{E} 8}{=} \overline{\mathrm{Q}}_{s}(\mathscr{M}(s \cdot))+\mathscr{M}^{a, b}(s)-\mathscr{M}(s) \leq \mathscr{M}^{a, b}(s),
$$

where we were allowed to use E8 because $\mathscr{M}(s)<+\infty$ as a consequence of $s \in\left[V_{k}^{a, b}, U_{k}^{a, b}\right)$ and the definitions of $V_{k}^{a, b}$ and $U_{k}^{a, b}$, and where the last step follows from $\mathscr{M}$ being a supermartingale and $0 \leq \mathscr{M}(s)<+\infty$. Otherwise, if $s \notin\left[V_{k}^{a, b}, U_{k}^{a, b}\right)$ for all $k \in \mathbb{N}$, we have that $\overline{\mathrm{Q}}_{s}\left(\mathscr{M}^{a, b}(s \cdot)\right)=\overline{\mathrm{Q}}_{s}\left(\mathscr{M}^{a, b}(s)\right)=\mathscr{M}^{a, b}(s)$, where we have usedE7 for the last inequality. Hence, we have that $\overline{\mathrm{Q}}_{s}\left(\mathscr{M}^{a, b}(s \cdot)\right) \leq \mathscr{M}^{a, b}(s)$ for all $s \in \mathscr{X}^{*}$, and we can therefore infer that $\mathscr{M}^{a, b}$ is indeed a non-negative supermartingale. 
Let us now show that $\mathscr{M}^{a, b}$ converges to $+\infty$ on all paths $\omega \in \Gamma(t)$ for which Equation A.2 holds. Consider such a path $\omega$. First, it follows from liminf $\mathscr{M}(\omega)<a$ that there exists some $n_{1} \in \mathbb{N}_{0}$ such that $\omega^{n_{1}} \sqsupseteq t$ and $\mathscr{M}\left(\omega^{n_{1}}\right)<a$. Take the first such $n_{1}$. Then it follows from the definition of $V_{1}^{a, b}$ that $\omega^{n_{1}} \in V_{1}^{a, b}$. Next, it follows from limsup $\mathscr{M}(\omega)>b$ that there exists some $m_{1} \in \mathbb{N}_{0}$ for which $m_{1}>n_{1}$ and $\mathscr{M}\left(\omega^{m_{1}}\right)>b$. Take the first such $m_{1}$. Then it follows from the definition of $U_{1}^{a, b}$ that $\omega^{m_{1}} \in U_{1}^{a, b}$. Repeating similar arguments over and over again allows us to conclude that $\omega$ goes through all the cuts $V_{1}^{a, b} \sqsubset U_{1}^{a, b} \sqsubset V_{2}^{a, b} \sqsubset \cdots \sqsubset V_{k}^{a, b} \sqsubset U_{k}^{a, b} \sqsubset \cdots$. For all $n>n_{1}$, let $k_{n} \in \mathbb{N}$ be the index such that $V_{k_{n}}^{a, b} \sqsubset \omega^{n}$ and $V_{k_{n}+1}^{a, b} \not \subset \omega^{n}$. Note that $k_{n} \rightarrow+\infty$ for $n \rightarrow+\infty$. Now, if $V_{k_{n}}^{a, b} \sqsubset \omega^{n}$ and $U_{k_{n}}^{a, b} \not \subset \omega^{n}$ for some $n>n_{1}$, then we use Equation A.4 to see that $\mathscr{M}^{a, b}\left(\omega^{n}\right)-\mathscr{M}^{a, b}(t)$ is bounded below by $\left(k_{n}-1\right)(b-a)+\mathscr{M}\left(\omega^{n}\right)-a \geq\left(k_{n}-1\right)(b-a)-a$ [ $\mathscr{M}$ is non-negative]. If on the other hand $U_{k_{n}}^{a, b} \sqsubset \omega^{n}$ and $V_{k_{n}+1}^{a, b} \not \subset \omega^{n}$ for some $n>n_{1}$, then Equation A.5 implies that $\mathscr{M}^{a, b}\left(\omega^{n}\right)-\mathscr{M}^{a, b}(t)$ is bounded below by $k_{n}(b-a) \geq\left(k_{n}-1\right)(b-a)-a$. All together, $\mathscr{M}^{a, b}\left(\omega^{n}\right)-\mathscr{M}^{a, b}(t)$ is bounded below by $\left(k_{n}-1\right)(b-a)-a$ for all $n>n_{1}$, which implies that

$$
\lim _{n \rightarrow+\infty}\left(\mathscr{M}^{a, b}\left(\omega^{n}\right)-\mathscr{M}^{a, b}(t)\right) \geq \lim _{n \rightarrow+\infty}\left(k_{n}-1\right)(b-a)-a=+\infty,
$$

because $\lim _{n \rightarrow+\infty} k_{n}=+\infty$ and $(b-a)>0$. This also implies that $\lim _{n \rightarrow+\infty} \mathscr{M}^{a, b}\left(\omega^{n}\right)=+\infty$ because $\mathscr{M}^{a, b}(t)=$ $\mathscr{M}(t)=1$.

We now use the countable set of rational couples $K:=\left\{(a, b) \in \mathbb{Q}^{2}: 0<a<b\right\}$ to define the process $\mathscr{M}^{*}$ :

$$
\mathscr{M}^{*}:=\sum_{(a, b) \in K} w^{a, b} \mathscr{M}^{a, b}
$$

with coefficients $w^{a, b}>0$ that sum to 1 . Hence, $\mathscr{M}^{*}$ is a countable convex combination of the non-negative supermartingales $\mathscr{M}^{a, b}$. By Lemma12 $\mathscr{M}^{*}$ is then a non-negative supermartingale. It is moreover clear that $\mathscr{M}^{*}(t)=\mathscr{M}(t)=1$, implying, together with its non-negativity, that $\mathscr{M}^{*}$ is a $t$-test supermartingale. We now show that $\mathscr{M}^{*}$ converges in the desired way as described by the proposition.

If $\mathscr{M}$ does not converge to an extended real number on some path $\omega \in \Gamma(t)$, then $\liminf \mathscr{M}(\omega)<\lim \sup \mathscr{M}(\omega)$. Since liminf $\mathscr{M}(\omega) \geq \inf _{s \in \mathscr{X}^{*}} \mathscr{M}(s) \geq 0$, there is at least one couple $\left(a^{\prime}, b^{\prime}\right) \in K$ such that liminf $\mathscr{M}(\omega)<a^{\prime}<b^{\prime}<$ limsup $\mathscr{M}(\omega)$, and as a consequence $\mathscr{M}^{a^{\prime}, b^{\prime}}$ converges to $+\infty$ on $\omega$. Then also $\lim w^{a^{\prime}, b^{\prime}} \mathscr{M}^{a^{\prime}, b^{\prime}}(\omega)=+\infty$ since $w^{a^{\prime}, b^{\prime}}>0$. For all other couples $(a, b) \in K \backslash\left\{\left(a^{\prime}, b^{\prime}\right)\right\}$, we have that $w^{a, b} \mathscr{M}^{a, b}$ is non-negative, so $\mathscr{M}^{*}$ indeed converges to $+\infty$ on $\omega$.

Finally, we show that $\mathscr{M}^{*}$ converges in $\overline{\mathbb{R}}$ on every path $\omega \in \Gamma(t)$ where $\mathscr{M}$ converges to a real number. Fix any such $\omega \in \Gamma(t)$. Then for any $\epsilon \in \mathbb{R}_{>0}$, there is an $n^{*} \in \mathbb{N}_{0}$ such that, for all $\ell \geq n \geq n^{*},\left|\mathscr{M}\left(\omega^{\ell}\right)-\mathscr{M}\left(\omega^{n}\right)\right| \leq \epsilon$ and therefore $\mathscr{M}\left(\omega^{\ell}\right)-\mathscr{M}\left(\omega^{n}\right) \geq-\epsilon$. Now fix any couple of rational numbers $0<a<b$ and, for any $i \in \mathbb{N}$, let $v_{i}^{\omega}$ and $u_{i}^{\omega}$ be the situations in respectively $V_{i}^{a, b}$ and $U_{i}^{a, b}$ where $\omega$ passes through [if it passes through these cuts]. We prove that $\mathscr{M}^{a, b}\left(\omega^{\ell}\right)-\mathscr{M}^{a, b}\left(\omega^{n}\right) \geq-2 \epsilon$ for any $\ell \geq n \geq n^{*}$. To do so, let us distinguish the following four cases:

- $V_{1}^{a, b} \not \subset \omega^{n}$ or $U_{k}^{a, b} \sqsubset \omega^{n} \not \supset V_{k+1}^{a, b}$ for some $k \in \mathbb{N}$ and moreover, $V_{1}^{a, b} \not \subset \omega^{\ell}$ or $U_{k^{\prime}}^{a, b} \sqsubset \omega^{\ell} \not \supset V_{k^{\prime}+1}^{a, b}$ for some $k^{\prime} \in \mathbb{N}$. 
Using Equations (A.3) and (A.5) for both $\omega^{n}$ and $\omega^{\ell}$, we get that

$$
\begin{aligned}
\mathscr{M}^{a, b}\left(\omega^{\ell}\right)-\mathscr{M}^{a, b}\left(\omega^{n}\right) & =\left[\mathscr{M}^{a, b}\left(\omega^{\ell}\right)-\mathscr{M}^{a, b}(t)\right]-\left[\mathscr{M}^{a, b}\left(\omega^{n}\right)-\mathscr{M}^{a, b}(t)\right] \\
& =\sum_{i=1}^{k^{\prime}}\left[\mathscr{M}\left(u_{i}^{\omega}\right)-\mathscr{M}\left(v_{i}^{\omega}\right)\right]-\sum_{i=1}^{k}\left[\mathscr{M}\left(u_{i}^{\omega}\right)-\mathscr{M}\left(v_{i}^{\omega}\right)\right],
\end{aligned}
$$

where we assume $k^{\prime}=0$ if $V_{1}^{a, b} \not \subset \omega^{\ell}$ and $k=0$ if $V_{1}^{a, b} \not \subset \omega^{n}$. Since $k^{\prime} \geq k$ [because $n \leq \ell$ and therefore $\omega^{n} \sqsubseteq \omega^{\ell}$ ] and $\mathscr{M}\left(u_{i}^{\omega}\right)-\mathscr{M}\left(v_{i}^{\omega}\right)>b-a>0$ for all $i \in \mathbb{N}$, we have that $\mathscr{M}^{a, b}\left(\omega^{\ell}\right)-\mathscr{M}^{a, b}\left(\omega^{n}\right) \geq 0>-2 \epsilon$ [where we also implicitly use the convention that $+\infty-\infty=+\infty]$.

- $V_{1}^{a, b} \not \subset \omega^{n}$ or $U_{k}^{a, b} \sqsubset \omega^{n} \not \supset V_{k+1}^{a, b}$ for some $k \in \mathbb{N}$ and moreover, $V_{k^{\prime}}^{a, b} \sqsubset \omega^{\ell} \not \supset U_{k^{\prime}}^{a, b}$ for some $k^{\prime} \in \mathbb{N}$. Using Equations (A.3) and A.5) for $\omega^{n}$ and Equation A.4 for $\omega^{\ell}$, we find that

$$
\begin{aligned}
\mathscr{M}^{a, b}\left(\omega^{\ell}\right)-\mathscr{M}^{a, b}\left(\omega^{n}\right) & =\left[\mathscr{M}^{a, b}\left(\omega^{\ell}\right)-\mathscr{M}^{a, b}(t)\right]-\left[\mathscr{M}^{a, b}\left(\omega^{n}\right)-\mathscr{M}^{a, b}(t)\right] \\
& =\left[\sum_{i=1}^{k^{\prime}-1}\left[\mathscr{M}\left(u_{i}^{\omega}\right)-\mathscr{M}\left(v_{i}^{\omega}\right)\right]+\mathscr{M}\left(\omega^{\ell}\right)-\mathscr{M}\left(v_{k^{\prime}}^{\omega}\right)\right]-\sum_{i=1}^{k}\left[\mathscr{M}\left(u_{i}^{\omega}\right)-\mathscr{M}\left(v_{i}^{\omega}\right)\right],
\end{aligned}
$$

where we assume $k=0$ if $V_{1}^{a, b} \not \subset \omega^{n}$. Note that $k^{\prime} \geq k+1$, because $k^{\prime} \geq k$ [since $\omega^{n} \sqsubseteq \omega^{\ell}$ ] and $k^{\prime}=k$ is impossible. Indeed, if $k=0, k^{\prime}=k$ is impossible because $k^{\prime} \in \mathbb{N}$. Otherwise, if $k>0, k^{\prime}=k$ would imply that $U_{k^{\prime}}^{a, b}=U_{k}^{a, b} \sqsubset \omega^{n} \sqsubseteq \omega^{\ell}$, contradicting the assumption that $\omega^{\ell} \not \supset U_{k^{\prime}}^{a, b}$. Hence, taking into account that $\mathscr{M}\left(u_{i}^{\omega}\right)-\mathscr{M}\left(v_{i}^{\omega}\right)>b-a>0$ for all $i \in \mathbb{N}$, we infer from A.7 that $\mathscr{M}^{a, b}\left(\omega^{\ell}\right)-\mathscr{M}^{a, b}\left(\omega^{n}\right) \geq \mathscr{M}\left(\omega^{\ell}\right)-\mathscr{M}\left(v_{k^{\prime}}^{\omega}\right)$ [again, also using the convention that $+\infty-\infty=+\infty$ ]. Finally, observe that $\omega^{n^{*}} \sqsubseteq \omega^{n} \sqsubseteq v_{k+1}^{\omega} \sqsubseteq v_{k^{\prime}}^{\omega} \sqsubset \omega^{\ell}$-the situation $v_{k+1}^{\omega}$ exists because $V_{k+1}^{a, b} \sqsubseteq V_{k^{\prime}}^{a, b} \sqsubset \omega^{\ell}$-and therefore, recalling how $n^{*}$ was chosen,

$$
\mathscr{M}^{a, b}\left(\omega^{\ell}\right)-\mathscr{M}^{a, b}\left(\omega^{n}\right) \geq \mathscr{M}\left(\omega^{\ell}\right)-\mathscr{M}\left(v_{k^{\prime}}^{\omega}\right) \geq-\epsilon \geq-2 \epsilon .
$$

- $V_{k}^{a, b} \sqsubset \omega^{n} \not \supset U_{k}^{a, b}$ for some $k \in \mathbb{N}$ and $U_{k^{\prime}}^{a, b} \sqsubset \omega^{\ell} \not \supset V_{k^{\prime}+1}^{a, b}$ for some $k^{\prime} \in \mathbb{N}$ [we automatically have that $V_{1}^{a, b} \sqsubset \omega^{\ell}$ because $V_{k}^{a, b} \sqsubset \omega^{n} \sqsubseteq \omega^{\ell}$ ]. Using Equation [A.4] for $\omega^{n}$ and Equation [A.5) for $\omega^{\ell}$, we get that

$$
\begin{aligned}
\mathscr{M}^{a, b}\left(\omega^{\ell}\right)-\mathscr{M}^{a, b}\left(\omega^{n}\right) & =\sum_{i=1}^{k^{\prime}}\left[\mathscr{M}\left(u_{i}^{\omega}\right)-\mathscr{M}\left(v_{i}^{\omega}\right)\right]-\left[\sum_{i=1}^{k-1}\left[\mathscr{M}\left(u_{i}^{\omega}\right)-\mathscr{M}\left(v_{i}^{\omega}\right)\right]+\mathscr{M}\left(\omega^{n}\right)-\mathscr{M}\left(v_{k}^{\omega}\right)\right] \\
& =\sum_{i=1}^{k^{\prime}}\left[\mathscr{M}\left(u_{i}^{\omega}\right)-\mathscr{M}\left(v_{i}^{\omega}\right)\right]-\left[\sum_{i=1}^{k-1}\left[\mathscr{M}\left(u_{i}^{\omega}\right)-\mathscr{M}\left(v_{i}^{\omega}\right)\right]+\mathscr{M}\left(\omega^{n}\right)\right]+\mathscr{M}\left(v_{k}^{\omega}\right) \\
& =\sum_{i=1}^{k^{\prime}}\left[\mathscr{M}\left(u_{i}^{\omega}\right)-\mathscr{M}\left(v_{i}^{\omega}\right)\right]-\sum_{i=1}^{k-1}\left[\mathscr{M}\left(u_{i}^{\omega}\right)-\mathscr{M}\left(v_{i}^{\omega}\right)\right]-\mathscr{M}\left(\omega^{n}\right)+\mathscr{M}\left(v_{k}^{\omega}\right),
\end{aligned}
$$

where the second step follows because $\mathscr{M}\left(v_{k}^{\omega}\right)$ is real [as a consequence of the definition of $V_{k}^{a, b}$ ] and the third step follows because $\sum_{i=1}^{k-1}\left[\mathscr{M}\left(u_{i}^{\omega}\right)-\mathscr{M}\left(v_{i}^{\omega}\right)\right] \geq 0$ [since all $\mathscr{M}\left(u_{i}^{\omega}\right)-\mathscr{M}\left(v_{i}^{\omega}\right)$ are positive] and $\mathscr{M}\left(\omega^{n}\right) \geq 0$ [because $\mathscr{M}$ is non-negative]. Using the fact that $k^{\prime} \geq k$ and that all $\mathscr{M}\left(u_{i}^{\omega}\right)-\mathscr{M}\left(v_{i}^{\omega}\right)$ are positive, the equation 
above implies that

$$
\begin{aligned}
\mathscr{M}^{a, b}\left(\omega^{\ell}\right)-\mathscr{M}^{a, b}\left(\omega^{n}\right) & \geq \sum_{i=k}^{k^{\prime}}\left[\mathscr{M}\left(u_{i}^{\omega}\right)-\mathscr{M}\left(v_{i}^{\omega}\right)\right]-\mathscr{M}\left(\omega^{n}\right)+\mathscr{M}\left(v_{k}^{\omega}\right) \\
& =\sum_{i=k+1}^{k^{\prime}}\left[\mathscr{M}\left(u_{i}^{\omega}\right)-\mathscr{M}\left(v_{i}^{\omega}\right)\right]+\mathscr{M}\left(u_{k}^{\omega}\right)-\mathscr{M}\left(\omega^{n}\right) \geq \mathscr{M}\left(u_{k}^{\omega}\right)-\mathscr{M}\left(\omega^{n}\right),
\end{aligned}
$$

where the equality follows from the fact that $\mathscr{M}\left(v_{k}^{(\omega)}\right)$ is real-valued and the last inequality follows once more from the positivity of all $\mathscr{M}\left(u_{i}^{\omega}\right)-\mathscr{M}\left(v_{i}^{(\omega)}\right)$. Then since $\omega^{n^{*}} \sqsubseteq \omega^{n} \sqsubseteq u_{k}^{\omega}$-the situation $u_{k}^{\omega}$ exists because $U_{k}^{a, b} \sqsubseteq$ $U_{k^{\prime}}^{a, b} \sqsubset \omega^{\ell}$-we infer from our assumptions about $n^{*}$ that

$$
\mathscr{M}^{a, b}\left(\omega^{\ell}\right)-\mathscr{M}^{a, b}\left(\omega^{n}\right) \geq \mathscr{M}\left(u_{k}^{\omega}\right)-\mathscr{M}\left(\omega^{n}\right) \geq-\epsilon \geq-2 \epsilon .
$$

- $V_{k}^{a, b} \sqsubset \omega^{n} \not \supset U_{k}^{a, b}$ for some $k \in \mathbb{N}$ and $V_{k^{\prime}}^{a, b} \sqsubset \omega^{\ell} \not \supset U_{k^{\prime}}^{a, b}$ for some $k^{\prime} \in \mathbb{N}$. Using Equation A.4 for both $\omega^{n}$ and $\omega^{\ell}$, we find that

$$
\begin{aligned}
\mathscr{M}^{a, b}\left(\omega^{\ell}\right)-\mathscr{M}^{a, b}\left(\omega^{n}\right) \\
=\left[\sum_{i=1}^{k^{\prime}-1}\left[\mathscr{M}\left(u_{i}^{\omega}\right)-\mathscr{M}\left(v_{i}^{\omega}\right)\right]+\mathscr{M}\left(\omega^{\ell}\right)-\mathscr{M}\left(v_{k^{\prime}}^{\omega}\right)\right]-\left[\sum_{i=1}^{k-1}\left[\mathscr{M}\left(u_{i}^{\omega}\right)-\mathscr{M}\left(v_{i}^{\omega}\right)\right]+\mathscr{M}\left(\omega^{n}\right)-\mathscr{M}\left(v_{k}^{\omega}\right)\right] \\
=\sum_{i=1}^{k^{\prime}-1}\left[\mathscr{M}\left(u_{i}^{\omega}\right)-\mathscr{M}\left(v_{i}^{\omega}\right)\right]+\mathscr{M}\left(\omega^{\ell}\right)-\mathscr{M}\left(v_{k^{\prime}}^{\omega}\right)-\sum_{i=1}^{k-1}\left[\mathscr{M}\left(u_{i}^{\omega}\right)-\mathscr{M}\left(v_{i}^{\omega}\right)\right]-\mathscr{M}\left(\omega^{n}\right)+\mathscr{M}\left(v_{k}^{\omega}\right) \\
\geq \sum_{i=k}^{k^{\prime}-1}\left[\mathscr{M}\left(u_{i}^{\omega}\right)-\mathscr{M}\left(v_{i}^{\omega}\right)\right]+\mathscr{M}\left(\omega^{\ell}\right)-\mathscr{M}\left(v_{k^{\prime}}^{\omega}\right)-\mathscr{M}\left(\omega^{n}\right)+\mathscr{M}\left(v_{k}^{\omega}\right)
\end{aligned}
$$

where the two last steps follow in a similar way as before; first using the real-valuedness of $\mathscr{M}\left(v_{k}^{\omega}\right)$ and the non-negativity of both $\mathscr{M}\left(\omega^{n}\right)$ and $\sum_{i=1}^{k-1}\left[\mathscr{M}\left(u_{i}^{\omega}\right)-\mathscr{M}\left(v_{i}^{\omega}\right)\right]$, and then using the fact that $k^{\prime} \geq k$ and that all $\mathscr{M}\left(u_{i}^{\omega}\right)-\mathscr{M}\left(v_{i}^{\omega}\right)$ are positive. If $k^{\prime}=k$, and therefore $\mathscr{M}\left(v_{k^{\prime}}^{\omega}\right)=\mathscr{M}\left(v_{k}^{\omega}\right) \in \mathbb{R}$, it follows from the expression above that $\mathscr{M}^{a, b}\left(\omega^{\ell}\right)-\mathscr{M}^{a, b}\left(\omega^{n}\right) \geq \mathscr{M}\left(\omega^{\ell}\right)-\mathscr{M}\left(\omega^{n}\right) \geq-\epsilon \geq-2 \epsilon$. Otherwise, if $k^{\prime}>k$, then we use the realvaluedness of $\mathscr{M}\left(v_{k}^{\omega}\right)$ to deduce from the expression above that

$$
\begin{aligned}
\mathscr{M}^{a, b}\left(\omega^{\ell}\right)-\mathscr{M}^{a, b}\left(\omega^{n}\right) & \geq \sum_{i=k+1}^{k^{\prime}-1}\left[\mathscr{M}\left(u_{i}^{\omega}\right)-\mathscr{M}\left(v_{i}^{\omega}\right)\right]+\mathscr{M}\left(\omega^{\ell}\right)-\mathscr{M}\left(v_{k^{\prime}}^{\omega}\right)+\mathscr{M}\left(u_{k}^{\omega}\right)-\mathscr{M}\left(\omega^{n}\right) \\
& \geq \mathscr{M}\left(\omega^{\ell}\right)-\mathscr{M}\left(v_{k^{\prime}}^{\omega}\right)+\mathscr{M}\left(u_{k}^{\omega}\right)-\mathscr{M}\left(\omega^{n}\right) \geq-2 \epsilon,
\end{aligned}
$$

where the last inequality follows from our assumptions about $n^{*}$ and the fact that $\omega^{n^{*}} \sqsubseteq \omega^{n} \sqsubseteq u_{k}^{\omega} \sqsubset v_{k^{\prime}}^{\omega} \sqsubset \omega^{\ell}$.

Hence, we conclude that for any $\epsilon \in \mathbb{R}_{>0}$, there is an $n^{*} \in \mathbb{N}_{0}$ such that $\mathscr{M}^{a, b}\left(\omega^{\ell}\right)-\mathscr{M}^{a, b}\left(\omega^{n}\right) \geq-2 \epsilon$ for all $\ell \geq n \geq n^{*}$ and any couple of rational numbers $0<a<b$.

To see that this implies that $\mathscr{M}^{*}$ converges to an extended real number on $\omega$, assume ex absurdo that it does not. Then there is some $\epsilon \in \mathbb{R}_{>0}$ such that $\liminf \mathscr{M}^{*}(\omega)<\limsup \mathscr{M}^{*}(\omega)-2 \epsilon$. As proved above, there is an $n^{*}$ such that, for all $\ell \geq n \geq n^{*}$ and any couple of rational numbers $0<a<b, \mathscr{M}^{a, b}\left(\omega^{\ell}\right)-\mathscr{M}^{a, b}\left(\omega^{n}\right) \geq-2 \epsilon$ and 
therefore also $\mathscr{M}^{a, b}\left(\omega^{\ell}\right) \geq \mathscr{M}^{a, b}\left(\omega^{n}\right)-2 \epsilon$. Then it follows directly from the definition of $\mathscr{M}^{*}$ that also $\mathscr{M}^{*}\left(\omega^{\ell}\right) \geq$ $\mathscr{M}^{*}\left(\omega^{n}\right)-2 \epsilon$ for all $\ell \geq n \geq n^{*}$. However, this is in contradiction with $\liminf \mathscr{M}^{*}(\omega)<\lim \sup \mathscr{M}^{*}(\omega)-2 \epsilon$, because the latter would require that there is some couple $\ell \geq n \geq n^{*}$ such that $\mathscr{M}^{*}\left(\omega^{\ell}\right)<\mathscr{M}^{*}\left(\omega^{n}\right)-2 \epsilon$. Hence, $\mathscr{M}^{*}$ converges to an extended real number on $\omega$.

Proof of Theorem 18, Due to Proposition 17 there is an $s$-test supermartingale $\mathscr{M}^{*}$ that converges to $+\infty$ on every path $\omega \in \Gamma(s)$ where $\mathscr{M}$ does not converge to an extended real number. Let $B \in \mathbb{R}$ be a lower bound for $\mathscr{M}$ and let $\mathscr{M}^{\prime}:=\frac{1}{\mathscr{M}(s)-B+1}\left(\mathscr{M}-B+\mathscr{M}^{*}\right)$. Since both $\mathscr{M}^{*}$ and $\mathscr{M}-B$ are supermartingales [because of E8], Lemma12 implies that $\left(\mathscr{M}-B+\mathscr{M}^{*}\right)$ is a supermartingale too and therefore, since $1 \leq \mathscr{M}(s)-B+1<+\infty$ [ $\mathscr{M}(s)$ is real],E9]implies that $\mathscr{M}^{\prime}$ is a supermartingale. Moreover, $\mathscr{M}^{\prime}$ is non-negative because both $\mathscr{M}-B$ and $\mathscr{M}^{*}$ are non-negative and $\mathscr{M}(s)-B+1 \geq 1$ which, together with $\mathscr{M}^{\prime}(s)=1$, allows us to conclude that $\mathscr{M}^{\prime}$ is an $s$-test supermartingale. Furthermore, consider any path $\omega \in \Gamma(s)$ such that $\mathscr{M}\left(\omega^{n}\right)$ does not converge to a real number. Then either it converges to $+\infty$ or it does not converge in $\overline{\mathbb{R}}$ [because $\mathscr{M}$ is bounded below]. In the first case, it follows from the non-negativity of $\mathscr{M}^{*}$ and the positivity of $\frac{1}{\mathscr{M}(s)-B+1}$ that $\mathscr{M}^{\prime}$ also converges to $+\infty$ on $\omega$. If $\mathscr{M}\left(\omega^{n}\right)$ does not converge in $\overline{\mathbb{R}}$, then $\mathscr{M}^{*}$ converges to $+\infty$ on $\omega$ and therefore, because $\mathscr{M}-B$ is non-negative and $\frac{1}{\mathscr{M}(s)-B+1}$ is positive, $\mathscr{M}^{\prime}$ also converges to $+\infty$ on $\omega$. All together, we have that $\mathscr{M}^{\prime}$ is an $s$-test supermartingale that converges to $+\infty$ on every path $\omega \in \Gamma(s)$ where $\mathscr{M}$ does not converge to a real number.

Proof of Theorem 19. Since $\overline{\mathrm{E}}_{\mathrm{V}}(\cdot \mid t)$ satisfies $\overline{\mathrm{V} 6}$ for any $t \in \mathscr{X}^{*}$, we have, for any $c \in \mathbb{R}$ and any $\omega \in \Omega$, that $\liminf _{n \rightarrow+\infty} \overline{\mathrm{E}}_{\mathrm{V}}\left(f \mid \omega^{n}\right) \geq f(\omega)$ if and only if $\liminf _{n \rightarrow+\infty} \overline{\mathrm{E}}_{\mathrm{V}}\left(f+c \mid \omega^{n}\right) \geq f(\omega)+c$. Therefore, and because $\mathrm{f}$ is bounded below, we can assume without loss of generality that $f$ is a global extended real variable such that $\inf f>0$.

We now associate with any couple of rational numbers $0<a<b$ the following recursively constructed sequences of cuts $\left\{U_{k}^{a, b}\right\}_{k \in \mathbb{N}_{0}}$ and $\left\{V_{k}^{a, b}\right\}_{k \in \mathbb{N}}$. Let $U_{0}^{a, b}:=\{s\}$ and, for $k \in \mathbb{N}$,

1. let $V_{k}^{a, b}:=\left\{v \in \mathscr{X}^{*}: U_{k-1}^{a, b} \sqsubset v, \overline{\mathrm{E}}_{\mathrm{V}}(f \mid v)<a\right.$ and $\left.\left(\forall t \in\left(U_{k-1}^{a, b}, v\right)\right) \overline{\mathrm{E}}_{\mathrm{V}}(f \mid t) \geq a\right\}$;

2. if $V_{k}^{a, b}$ is non-empty, choose a positive supermartingale $\mathscr{M}_{k}^{a, b} \in \overline{\mathbb{M}}_{\mathrm{b}}$ such that $\mathscr{M}_{k}^{a, b}(v)<a$ and liminf $\mathscr{M}_{k}^{a, b} \geq_{v}$ $f$ for all $v \in V_{k}^{a, b}$, and let $U_{k}^{a, b}:=\left\{u \in \mathscr{X}^{*}: V_{k}^{a, b} \sqsubset u: \mathscr{M}_{k}^{a, b}(u)>b\right.$ and $\left.\left(\forall t \in\left(V_{k}^{a, b}, u\right)\right) \mathscr{M}_{k}^{a, b}(t) \leq b\right\}$;

3. if $V_{k}^{a, b}$ is empty, let $U_{k}^{a, b}:=\emptyset$.

Note that all $U_{k}^{a, b}$ and all $V_{k}^{a, b}$ are indeed (partial or complete) cuts. We now first show that, if $V_{k}^{a, b}$ is non-empty, there always is a supermartingale $\mathscr{M}_{k}^{a, b}$ that satisfies the conditions above. We infer from the definition of the cut $V_{k}^{a, b}$ that

$$
\inf \left\{\mathscr{M}(v): \mathscr{M} \in \overline{\mathbb{M}}_{\mathrm{b}} \text { and } \liminf \mathscr{M} \geq_{v} f\right\}=\overline{\mathrm{E}}_{\mathrm{V}}(f \mid v)<a \text { for all } v \in V_{k}^{a, b} .
$$

So, for all $v \in V_{k}^{a, b}$, we can choose a supermartingale $\mathscr{M}_{k, v}^{a, b}$ such that $\mathscr{M}_{k, v}^{a, b}(v)<a$ and $\liminf \mathscr{M}_{k, v}^{a, b} \geq_{v} f$. Consider now the extended real process $\mathscr{M}_{k}^{a, b}$ defined, for all $t \in \mathscr{X}^{*}$, by

$$
\mathscr{M}_{k}^{a, b}(t):= \begin{cases}\mathscr{M}_{k, v}^{a, b}(t) & \text { if } v \sqsubseteq t \text { for some } v \in V_{k}^{a, b} ; \\ a & \text { otherwise. }\end{cases}
$$


It is clear that $\mathscr{M}_{k}^{a, b}(v)<a$ and $\liminf \mathscr{M}_{k}^{a, b} \geq_{v} f$ for all $v \in V_{k}^{a, b}$. We furthermore show that $\mathscr{M}_{k}^{a, b}$ is a positive supermartingale.

It follows from Lemma11 that, for all $v \in V_{k}^{a, b}$,

$$
\mathscr{M}_{k, v}^{a, b}(t) \geq \inf _{\omega \in \Gamma(t)} \liminf \mathscr{M}_{k, v}^{a, b}(\omega) \geq \inf _{\omega \in \Gamma(t)} f(\omega) \geq \inf f>0 \text { for all } t \sqsupseteq v .
$$

Since also $a>0$, it follows that $\mathscr{M}_{k}^{a, b}$ is positive. To show that $\overline{\mathrm{Q}}_{t}\left(\mathscr{M}_{k}^{a, b}(t \cdot)\right) \leq \mathscr{M}_{k}^{a, b}(t)$ for all $t \in \mathscr{X}^{*}$, fix any $t \in \mathscr{X}^{*}$ and consider two cases. If $V_{k}^{a, b} \sqsubseteq t$, then $\mathscr{M}_{k}^{a, b}(t)=\mathscr{M}_{k, v}^{a, b}(t)$ and $\mathscr{M}_{k}^{a, b}(t \cdot)=\mathscr{M}_{k, v}^{a, b}(t \cdot)$ for some $v \in V_{k}^{a, b}$, and therefore $\overline{\mathrm{Q}}_{t}\left(\mathscr{M}_{k}^{a, b}(t \cdot)\right)=\overline{\mathrm{Q}}_{t}\left(\mathscr{M}_{k, v}^{a, b}(t \cdot)\right) \leq \mathscr{M}_{k, v}^{a, b}(t)=\mathscr{M}_{k}^{a, b}(t)$. If $V_{k}^{a, b} \nsubseteq t$, then for any $x \in \mathscr{X}$ we either have that $t x \in V_{k}^{a, b}$, which implies that $\mathscr{M}_{k}^{a, b}(t x)<a$, or $V_{k}^{a, b} \nsubseteq t x$ and therefore $\mathscr{M}_{k}^{a, b}(t x)=a$. Hence, we infer that $\mathscr{M}_{k}^{a, b}(t \cdot) \leq a$, and therefore, byE4]andE7 that $\overline{\mathrm{Q}}_{t}\left(\mathscr{M}_{k}^{a, b}(t \cdot)\right) \leq a=\mathscr{M}_{k}^{a, b}(t)$. So we can conclude that $\mathscr{M}_{k}^{a, b}$ is a positive supermartingale.

Next, consider the extended real process $\mathscr{T}^{a, b}$ defined by $\mathscr{T}^{a, b}(t):=1$ for all $t \not \supset s$, and

$$
\mathscr{T}^{a, b}(t x):= \begin{cases}\mathscr{M}_{k}^{a, b}(t x) \mathscr{T}^{a, b}(t) / \mathscr{M}_{k}^{a, b}(t) & \text { if } t \in\left[V_{k}^{a, b}, U_{k}^{a, b}\right) \text { for some } k \in \mathbb{N} \\ \mathscr{T}^{a, b}(t) & \text { otherwise, }\end{cases}
$$

for all $t \sqsupseteq s$ and all $x \in \mathscr{X}$. We prove that this process is a positive $s$-test supermartingale that converges to $+\infty$ on all paths $\omega \in \Gamma(s)$ such that

$$
\liminf _{n \rightarrow+\infty} \overline{\mathrm{E}}_{\mathrm{V}}\left(f \mid \omega^{n}\right)<a<b<f(\omega)
$$

That $\mathscr{T}^{a, b}$ is well-defined follows from the fact that, for any $k \in \mathbb{N}$ and any $t \in\left[V_{k}^{a, b}, U_{k}^{a, b}\right), \mathscr{M}_{k}^{a, b}(t)$ is positive and moreover real because of the definition of $U_{k}^{a, b}$. The process $\mathscr{T}^{a, b}$ is also positive because, for any $k \in \mathbb{N}$ and any $t \in\left[V_{k}^{a, b}, U_{k}^{a, b}\right), \mathscr{M}_{k}^{a, b}(t)$ is real and positive and $\mathscr{M}_{k}^{a, b}(t \cdot)$ is positive, and therefore $\mathscr{M}_{k}^{a, b}(t \cdot) / \mathscr{M}_{k}^{a, b}(t)$ is positive. Furthermore, if $t \in\left[V_{k}^{a, b}, U_{k}^{a, b}\right)$ for some $k \in \mathbb{N}$, we have that

$$
\overline{\mathrm{Q}}_{t}\left(\mathscr{T}^{a, b}(t \cdot)\right)=\overline{\mathrm{Q}}_{t}\left(\mathscr{M}_{k}^{a, b}(t \cdot) \mathscr{T}^{a, b}(t) / \mathscr{M}_{k}^{a, b}(t)\right)^{\mathrm{EgEE}}=\underline{\underline{\mathrm{E}} 33}-\overline{\mathrm{Q}}_{t}\left(\mathscr{M}_{k}^{a, b}(t \cdot)\right) \mathscr{T}^{a, b}(t) / \mathscr{M}_{k}^{a, b}(t) \leq \mathscr{T}^{a, b}(t),
$$

where the second step also uses the fact that $\mathscr{M}_{k}^{a, b}(t \cdot)$ and $\mathscr{T}^{a, b}(t) / \mathscr{M}_{k}^{a, b}(t)$ are positive [since, as we have shown above, $\mathscr{M}_{k}^{a, b}(t)$ is real and positive, and $\mathscr{T}^{a, b}(t)$ is positive], and where the last step uses the supermartingale character of $\mathscr{M}_{k}^{a, b}$ together with the fact that $\mathscr{T}^{a, b}(t) / \mathscr{M}_{k}^{a, b}(t)$ is positive. Otherwise, if $t \notin\left[V_{k}^{a, b}, U_{k}^{a, b}\right)$ for all $k \in \mathbb{N}$, then $\overline{\mathrm{Q}}_{t}\left(\mathscr{T}^{a, b}(t \cdot)\right)=\overline{\mathrm{Q}}_{t}\left(\mathscr{T}^{a, b}(t)\right)=\mathscr{T}^{a, b}(t)$ because of E7, Hence, we have that $\overline{\mathrm{Q}}_{t}\left(\mathscr{T}^{a, b}(t \cdot)\right) \leq \mathscr{T}^{a, b}(t)$ for all $t \in \mathscr{X}^{*}$, which together with the fact that $\mathscr{T}^{a, b}(s)=1$, allows us to conclude that $\mathscr{T}^{a, b}$ is indeed a positive $s$-test supermartingale.

Next, we show that $\mathscr{T}^{a, b}$ converges to $+\infty$ on all paths $\omega \in \Gamma(s)$ for which (A.10) holds. Consider such a path $\omega$. Then $\omega$ goes through all the cuts $U_{0}^{a, b} \sqsubset V_{1}^{a, b} \sqsubset U_{1}^{a, b} \sqsubset \ldots \sqsubset V_{k}^{a, b} \sqsubset U_{k}^{a, b} \sqsubset \ldots$. Indeed, it is trivial that $\omega$ goes through $U_{0}^{a, b}=\{s\}$. Furthermore, it follows from $\liminf _{n \rightarrow+\infty} \overline{\mathrm{E}}_{\mathrm{V}}\left(f \mid \omega^{n}\right)<a$ that there is an $n_{1} \in \mathbb{N}$ such that $\omega^{n_{1}} \sqsupset s$ and $\overline{\mathrm{E}}_{\mathrm{V}}\left(f \mid \omega^{n_{1}}\right)<a$. Take the first such $n_{1} \in \mathbb{N}$. Then it follows from the definition of $V_{1}^{a, b}$ that $\omega^{n_{1}} \in V_{1}^{a, b}$. Next, it follows from $\liminf _{n \rightarrow+\infty} \mathscr{M}_{1}^{a, b}\left(\omega^{n}\right) \geq f(\omega)>b$ that there exists some $m_{1} \in \mathbb{N}$ for which $m_{1}>n_{1}$ and $\mathscr{M}_{1}^{a, b}\left(\omega^{m_{1}}\right)>b$. Take the first such $m_{1}$. Then it follows from the definition of $U_{1}^{a, b}$ that $\omega^{m_{1}} \in U_{1}^{a, b}$. 
Repeating similar arguments over and over again allows us to conclude that $\omega$ indeed goes through all the cuts $U_{0}^{a, b} \sqsubset V_{1}^{a, b} \sqsubset U_{1}^{a, b} \sqsubset \ldots \sqsubset V_{k}^{a, b} \sqsubset U_{k}^{a, b} \sqsubset \ldots$.

In what follows, we use the following notation. For any $k \in \mathbb{N}_{0}$, let $u_{k}^{\omega}$ be the (necessarily unique) situation in $U_{k}^{a, b}$ where $\omega$ goes through. Similarly, for any $k \in \mathbb{N}$, let $v_{k}^{\omega}$ be the (necessarily unique) situation in $V_{k}^{a, b}$ where $\omega$ goes through. For all $n \in \mathbb{N}_{0}$, let $k_{n} \in \mathbb{N}_{0}$ be defined by $k_{n}:=0$ if $V_{1}^{a, b} \not \subset \omega^{n}$ and otherwise, let $k_{n}$ be such that $V_{k_{n}}^{a, b} \sqsubset \omega^{n}$ and $V_{k_{n}+1}^{a, b} \not \subset \omega^{n}$. Note that $k_{n} \rightarrow+\infty$ for $n \rightarrow+\infty$ because $\omega$ goes through all the cuts $V_{1}^{a, b} \sqsubset V_{2}^{a, b} \sqsubset \ldots \sqsubset V_{k}^{a, b} \sqsubset \ldots$. For any $n \in \mathbb{N}_{0}$ such that $k_{n} \geq 1$, we now have one of the following two cases:

1. The first case is that $\omega^{n} \in\left(V_{k_{n}}^{a, b}, U_{k_{n}}^{a, b}\right.$. Then by applying Equation $\mathbf{A . 9}$ for each subsequent step and cancelling out the intermediate terms, which is possible because $\mathscr{M}_{\ell}^{a, b}\left(s^{\prime}\right)$ is real for any $s^{\prime} \in\left[V_{\ell}^{a, b}, U_{\ell}^{a, b}\right)$ and any $\ell \in \mathbb{N}$ [this follows readily from the definition of the cuts $V_{\ell}^{a, b}$ and $U_{\ell}^{a, b}$ ], we find that

$$
\mathscr{T}^{a, b}\left(\omega^{n}\right)=\left(\prod_{\ell=1}^{k_{n}-1} \frac{\mathscr{M}_{\ell}^{a, b}\left(u_{\ell}^{\omega}\right)}{\mathscr{M}_{\ell}^{a, b}\left(v_{\ell}^{\omega}\right)}\right) \frac{\mathscr{M}_{k_{n}}^{a, b}\left(\omega^{n}\right)}{\mathscr{M}_{k_{n}}^{a, b}\left(v_{k_{n}}^{\omega}\right)} .
$$

Since $\mathscr{M}_{k_{n}}^{a, b}\left(\omega^{n}\right) \geq \inf f>0$ [due to Equation [A.8)], $\mathscr{M}_{\ell}^{a, b}\left(u_{\ell}^{\omega}\right)>b>0$ for all $\ell \in\left\{1, \ldots, k_{n}-1\right\}$ and $0<$ $\mathscr{M}_{\ell}^{a, b}\left(v_{\ell}^{\omega}\right)<a$ for all $\ell \in\left\{1, \ldots, k_{n}\right\}$, we get that

$$
\mathscr{T}^{a, b}\left(\omega^{n}\right) \geq\left(\frac{b}{a}\right)^{k_{n}-1} \frac{\mathscr{M}_{k_{n}}^{a, b}\left(\omega^{n}\right)}{a} \geq\left(\frac{b}{a}\right)^{k_{n}-1}\left(\frac{\inf f}{a}\right) .
$$

2. The second case is that $\omega^{n} \in\left(U_{k_{n}}^{a, b}, V_{k_{n}+1}^{a, b}\right]$. Then, by repeatedly applying Equation [A.9), we have that

$$
\mathscr{T}^{a, b}\left(\omega^{n}\right)=\prod_{\ell=1}^{k_{n}} \frac{\mathscr{M}_{\ell}^{a, b}\left(u_{\ell}^{\omega}\right)}{\mathscr{M}_{\ell}^{a, b}\left(v_{\ell}^{\omega}\right)}
$$

Since $\mathscr{M}_{\ell}^{a, b}\left(u_{\ell}^{\omega}\right)>b>0$ and $0<\mathscr{M}_{\ell}^{a, b}\left(v_{\ell}^{(\omega)}\right)<a$ for all $\ell \in\left\{1, \ldots, k_{n}\right\}$, we find that

$$
\mathscr{T}^{a, b}\left(\omega^{n}\right)>\left(\frac{b}{a}\right)^{k_{n}}
$$

Since $\inf f>0, a>0$ and $\frac{b}{a}>1$, and since $\lim _{n \rightarrow+\infty} k_{n}=+\infty$, it follows from the two expressions above that indeed $\lim _{n \rightarrow+\infty} \mathscr{T}^{a, b}\left(\omega^{n}\right)=+\infty$.

To finish, we use the countable set of rational couples $K:=\left\{(a, b) \in \mathbb{Q}^{2}: 0<a<b\right\}$ to define the process $\mathscr{T}$ :

$$
\mathscr{T}:=\sum_{(a, b) \in K} w^{a, b} \mathscr{T}^{a, b},
$$

with coefficients $w^{a, b}>0$ that sum to 1 . Hence, $\mathscr{T}$ is a countable convex combination of the positive $s$-test supermartingales $\mathscr{T}^{a, b}$. By Lemma 12, $\mathscr{T}$ is then also a supermartingale. It is also positive, because all $w^{a, b} \mathscr{T}^{a, b}$ are positive. Since it is moreover clear that $\mathscr{T}(s)=1$, the process $\mathscr{T}$ is a positive $s$-test supermartingale. Furthermore, $\mathscr{T}$ converges to $+\infty$ on the paths $\omega \in \Gamma(s)$ where $\liminf _{n \rightarrow+\infty} \overline{\mathrm{E}}_{\mathrm{V}}\left(f \mid \omega^{n}\right)<f(\omega)$. Indeed, consider such a 
path $\omega$. Then since $f(\omega) \geq \inf f>0$, there is at least one couple $\left(a^{\prime}, b^{\prime}\right) \in K$ such that $\liminf _{n \rightarrow+\infty} \overline{\mathrm{E}}_{\mathrm{V}}\left(f \mid \omega^{n}\right)<$ $a^{\prime}<b^{\prime}<f(\omega)$, and as a consequence $\lim _{n \rightarrow+\infty} \mathscr{T}^{a^{\prime}, b^{\prime}}\left(\omega^{n}\right)=+\infty$. Then also $\lim _{n \rightarrow+\infty} w^{a^{\prime}, b^{\prime}} \mathscr{T}^{a^{\prime}, b^{\prime}}\left(\omega^{n}\right)=+\infty$, and since $w^{a, b} \mathscr{T}^{a, b}$ is positive for all other couples $(a, b) \in K \backslash\left(a^{\prime}, b^{\prime}\right)$, the positive $s$-test supermartingale $\mathscr{T}$ indeed converges to $+\infty$ on $\omega$. 